Portland State University

PDXScholar

Spring 6-8-2018

\title{
Evaluating the Effectiveness of Justice Reinvestment Legislation in Oregon: Analyses of State and County Implementation
}

Christopher Wade Dollar

Portland State University

Follow this and additional works at: https://pdxscholar.library.pdx.edu/open_access_etds

Part of the Criminal Procedure Commons, and the Criminology and Criminal Justice Commons Let us know how access to this document benefits you.

Recommended Citation

Dollar, Christopher Wade, "Evaluating the Effectiveness of Justice Reinvestment Legislation in Oregon: Analyses of State and County Implementation" (2018). Dissertations and Theses. Paper 4462.

https://doi.org/10.15760/etd.6346

This Thesis is brought to you for free and open access. It has been accepted for inclusion in Dissertations and Theses by an authorized administrator of PDXScholar. Please contact us if we can make this document more accessible: pdxscholar@pdx.edu. 
Evaluating the Effectiveness of Justice Reinvestment Legislation in Oregon:

Analyses of State and County Implementation

$$
\text { by }
$$

Christopher Wade Dollar

A thesis submitted in partial fulfillment of the requirements for the degree of

\author{
Master of Science \\ in \\ Criminology and Criminal Justice
}

Thesis Committee:

Christopher Campbell, Chair

Laura Hickman

Mark Harmon

Portland State University

2018 
C 2018 Christopher Wade Dollar 
Abstract

Sentencing reform and "tough on crime" policies have assisted in the inflation of the United States' prison population by nearly $400 \%$ over the last 50 years. In 2003 , justice reinvestment was conceptualized as a way to decrease recidivism and remedy the exorbitant correctional spending by reinvesting funds on rehabilitation and reentry assistance to those leaving custodial institutions. Early implementations of justice reinvestment in Connecticut and Texas achieved both savings and reductions in prison populations. This led to the creation of the Justice Reinvestment Initiative by the U.S. Bureau of Justice Assistance in 2010. Officials of the Justice Reinvestment Initiative sought states who were willing to achieve bi-partisan agreements on reform and reinvestment strategies to assist in the creation and implementation of this new policy. The State of Oregon began this process in early 2012 and completed the process with the enrollment of HB 3194 in July of 2013. Despite the implementation of this policy in 17 states, few evaluations have been performed on the effectiveness of justice reinvestment policy.

This study employs a quasi-experimental time series analysis of corrections data from the State of Oregon, the high usage county, medium usage county, and the low usage county proxies to assess the effectiveness of the law. Counties were selected as proxies for levels of justice reinvestment grant usage. These data include prison admissions (June 2010 - July 2016), probation admissions (June 2010 - July 2016), and the number of individuals on community supervision (July 2010 - December 2015). Analyses reveal significant changes in all measures. The results of this study have several implications for current and future implementations of justice reinvestment. 
Dedication

I would like to dedicate this work to my family, for always supporting me (even through years of rebellion). Specifically, to my father, for not giving up on my ability to do math, and for not turning the car around because I couldn't remember the formula for velocity. 
Acknowledgments

I would like to thank faculty and staff in the department of Criminology and Criminal Justice at Portland State University. The guidance, advice, and assistance I have received from each of you has been invaluable in navigating this graduate program and developing my skills as a professional. I will continue to reflect on these lessons as I move forward in my career.

I would like to directly thank my thesis committee: Dr. Laura Hickman, Dr. Mark Harmon, and Dr. Christopher Campbell.

Dr. Hickman, thank you for your critical evaluation and keen eye for policy/ political considerations. Your comments and input into this work have been indispensable.

Dr. Harmon, thank you for suggesting that I apply for this program and introducing me to Criminology and Criminal Justice. It has deepened my understanding of the world and motivated me to continue on with this work.

Dr. Campbell, I cannot thank you enough. Your support and guidance during these two years as my committee chairperson and advisor have meant the world. Despite all of the panic and worry, there is no one else that I would rather have had as a mentor. 
Table of Contents

Abstract

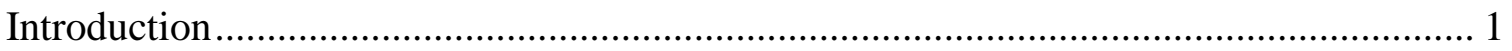

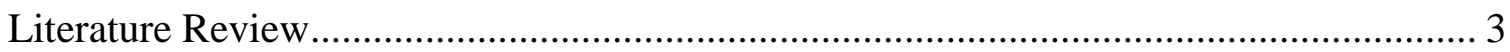

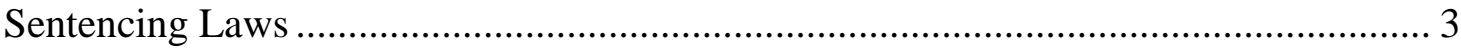

Justice Reinvestment ................................................................................... 7

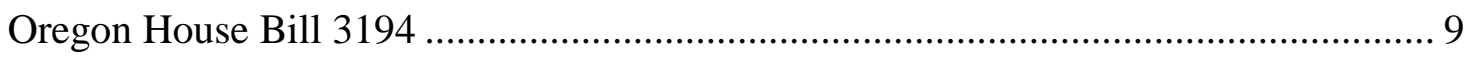

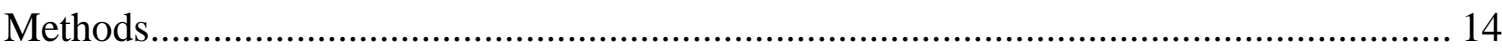

Time Series in Policy Research ......................................................................... 14

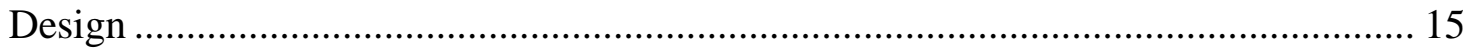

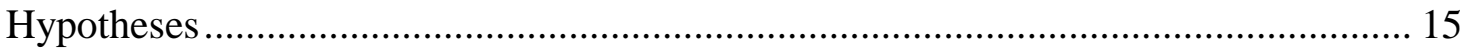

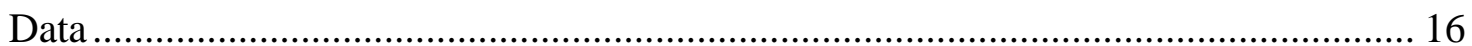

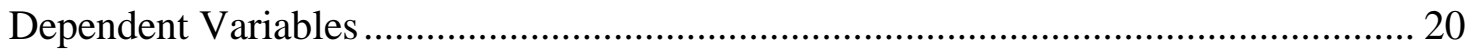

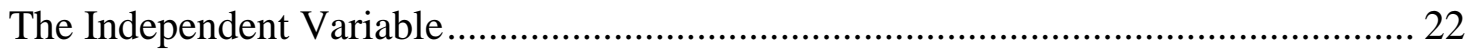

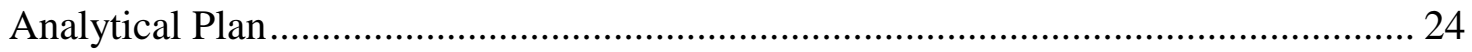

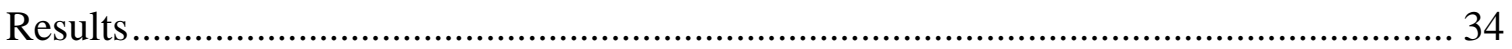

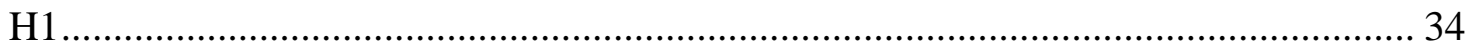

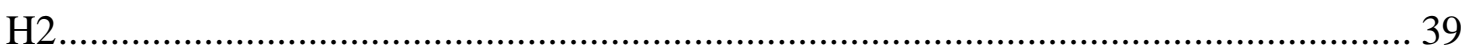

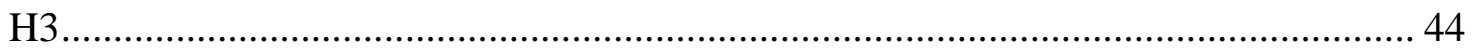

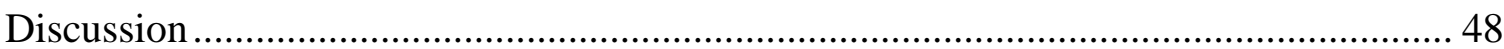

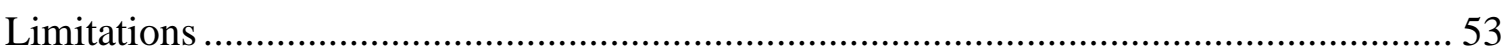

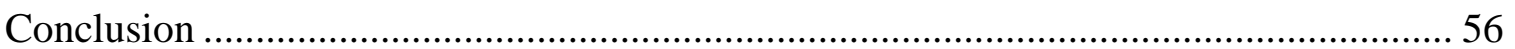

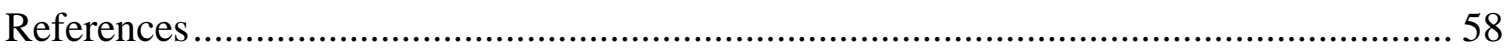

Appendix A - County Subtype and Populations ....................................................... 63

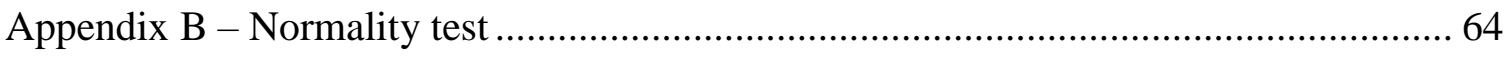

Appendix C - Descriptive Statistics by Year Within Study ....................................... 65

Appendix D - Figures of raw data, ACF plots, PACF, and GLS plots ......................... 67

Appendix E - Recidivism Example ...................................................................... 79 


\section{List of Tables}

Table 1: State of Oregon Descriptive Statistics by Year ..............................................32

Table 1.A: Yearly Mean Rates by Measure and Political Unit.......................................32

Table 2: ARIMA model Parameter Estimates and Q fit .............................................33

Table 3: Hypothesis 1, Regression Coefficients and Absolute \& Relative Change ..........36

Table 4: Hypothesis 2, Regression Coefficients and Absolute \& Relative Change ..........39

Table 5: Hypothesis 3, Regression Coefficients and Absolute \& Relative Change .........44 
List of Figures

Figure 1. State of Oregon Prison Admissions by Month ..................................................35

Figure 2. The High Usage Prison Admissions by Month ....................................................37

Figure 3. Oregon Probation Admissions by Month ........................................................40

Figure 4. The High Usage Probation Admissions by Month...............................................41

Figure 5. Medium Usage County Probation Admissions by Month...................................42

Figure 6. The High Usage Community Supervision Population .........................................45 
Introduction

Over the last 50 years the United States has witnessed an increase in institutional and community corrections and related budgets. Numerous governmental agencies and independent studies have reported that state and federal prison populations have increased by 500-700\% since the 1970's (BJA, 2014; Pettit \& Western, 2004; Sampson \& Loeffler, 2010; Roberts, 2004). According to current estimates by the U.S. Department of Justice, approximately 650,000 individuals are released from federal and state prisons each year. Of these, approximately 429,000 individuals eventually reenter prison (DOJ, 2017). Simultaneously, the accumulated costs of states' expenditures on corrections also inflated the national cost to over $\$ 51$ billion in 2012 (BJA, 2014). This revolving door of offenders has created a severe problem for the U.S. justice system. Mass incarceration is a byproduct of both federal and state policy changes between 1970 and 2000. These include the increase in determinant sentencing, truth-in-sentencing laws, the abolition of parole boards, the War on Drugs, "three strikes" laws, and "One Strike You're Out” housing policies. In response to the problematic and high costs of tough on crime policies, some state governments are seeking to reduce their prison populations and institutional budgets through the use of justice reinvestment which emerged in 2003.

Justice Reinvestment refers to policies that attempt to redirect offenders from prison to accumulate savings. Savings should then be invested in rehabilitation and reentry programs for offenders to reduce recidivism and overall corrections budgets. Due to early successes in Connecticut (2004), Texas (2007), and Kansas (2007) (BJA, 2014), this style of policy reform has now been expanded to 17 states. The expansion of justice reinvestment and the use of evidence-based practices (EBPs) is the outcome of the United States 
Congress creating the Justice Reinvestment Initiative (JRI) under the oversight of the Bureau of Justice Assistance (BJA, 2014) in 2010. One of the main features of JRI policy is the allocation of a portion of the reinvested funds into program evaluation; which has led to a growing number of studies examining specific programs to ensure a state's Departments of Corrections is utilizing EBPs (BJA, 2014). A large portion of research resources are devoted to program evaluations. Three percent of justice reinvestment funds are reserved for research on evaluation projects, and many of the studies receiving these funds are ongoing.

This study addresses this gap in research by analyzing Oregon's justice reinvestment bill HB 3194 (2013). HB 3194 (2013) decreased mandatory minimum sentences for several offenses, restructured transitional leave, and required agencies to report on program effectiveness and use of EBPs. By doing this, the legislature intended to decrease the use of prisons and jails by supporting offenders on probation and parole. Ideally, this should translate to a decrease in prison populations, an increase in community supervision populations, and a reduction in recidivism. The research question for this study is: Has HB 3194 (2013) achieved its desired goals and or outcomes? To date, no comprehensive analysis has been performed on these laws to evaluate their success in achieving intended outcomes. By utilizing interrupted time-series analyses methods to evaluate the bill's intended outcome measures this study examines the effectiveness of the legislation. 
Literature Review

Sentencing Laws

Calls for criminal justice reform have risen as a potential antidote for the continuous growth of both state and federal prison populations and correctional budgets. The penal harm movement began in the 1970's and is a particular example of this (Listwan et al., 2008). Shaped by the political and social turmoil of the decades prior (Simon, 2017, Monterosso, 2009), the penal harm movement developed from a multitude of factors related to enforcement and sentencing strategy. In 1968 presidential candidate Richard Nixon ran what is now known as the "southern strategy" to capture the votes of conservative democrats in the south. He campaigned on a "Law and Order" platform asking voters to reject the lawlessness of the social movements (Alexander, 2012; Thompson, 2010). In 1971 President Nixon advocated a "War on Drugs" stating that narcotics were "public enemy number one" and that it was "necessary to wage a new, all-out offensive" (Nixon, 1971). Nixon's call for get-tough legislation reflected a political movement which emerged as a response to perceived lawlessness known as popular punitivism - that is, the relationship between politics, the media, and the public to shape crime policy (Campbell, 2015; Monterosso, 2009).

Growth of popular punitivism and the tough-on-crime movement led to truth-insentencing (TIS) laws often mandating prisoners be incarcerated for an average (across states) of $85 \%$ of their sentence (Travis, Western \&, Redburn, 2014). Soon after, the discussion on rehabilitation and judicial discretion came to a head with Martinson's infamous 1974 publication insinuating that "nothing works" in correctional rehabilitation. The report called for a more enlightened scientific approach to studying rehabilitation 
programs (Martinson, 1974), yet became a rallying cry for "get-tough" sentiments (Cullen \& Gendreau, 2001).

These events resulted in the decline of indeterminate sentencing, which includes a judge-determined bracketed range for sentence length and a parole board that determines the release date (Marvell and Moody, 1996). The intent was largely to ensure "just deserts" for offenders (Shepherd, 2002). Truth-in-sentencing laws sought to increase the amount of time violent offenders serve (to $85 \%$ of sentence) by decreasing the discretion of parole boards and implementing determinant sentencing practices (Travis, Western \&, Redburn, 2014).

Determinate sentencing allocates the near exact length of prison time as determined by the judge without the use of parole boards, but early release is possible through good behavior credits (Marvel \& Moody, 1996). Recent studies of determinant sentencing and TIS laws reveal that while they do deter the crimes encapsulated in legislation, a criminogenic thermodynamic effect (Wright \& Rosky, 2011) is also present. The criminogenic thermodynamic effect occurs when offenders adapt by committing crimes not covered by the legislation (Shepherd, 2002). System thermodynamics is similar, where pressures (e.g. cases) placed on one part of the system are released (e.g. deciding not to prosecute) or create different pressures in other areas deeper into the system (Wright \& Rosky, 2011). These subtle policy changes increased the length of stay for offenders and restricted options for early release, which resulted in a long-term boost to the prison population. During this time, the number of incarcerated people in the United States increased from 198,800 in 1970 (Cahalan, 1986) to an estimated 503,600 in 1980 (Carson \& Anderson, 2016). 
In 1982, President Reagan perpetuated the political surge for tough-on-crime policies by escalating the Nixon administration's "War on Drugs" campaign. Mandatory minimum sentences for all drug offenses were introduced during this time (Alexander, 2012). The Anti-Drug Abuse Acts of 1986 and 1988 increased mandatory minimum sentences for drug offenses; other critical impacts on marginalized populations include residential evictions due to drug crimes ( $99^{\text {th }}$ Congress, 1986). The acts jointly had several other large effects. Federal funding for police forces to pursue drug offenses increased dramatically: in 1981 \$1.5 billion was spent, \$6.6 billion in 1989 (White House, 1992), and $\$ 15.25$ billion was requested by the Obama Administration in 2017 (White House, 2016). The use of incarceration for drug offenses has also increased. Approximately $60 \%$ of all federal prisoners were serving time for drug related offenses by 2000 , which is a substantial rise from the 6\% seen in 1979 (Pettit \& Western, 2004). The number of individuals being sentenced to prison for drug crimes in 1991 had increased nine times from 1980, and another ten times between 1991 and 2000 (Clear, 2009)

Unintended consequences of the war on drugs include the destruction of social cohesion in minority neighborhoods (Clear, 2009; Jensen et al., 2004; Hagan \& Coleman, 2001), thus posing a threat to the success of community supervision. Hagan \& Coleman (2001) argue that the destruction of social cohesion isolates large communities from the whole of society. As offenders are stripped of fundamental rights of citizenship the community slowly devolves into a prison-like condition. Emphasis on surveillance and supervision by police officers, probation and parole officers, and social workers causes each agent to function as prison guards (Hagain \& Coleman, 2001). Theories of deterrence argue that in order to successfully deter criminals, the system must create incapacitation 
and stigma that are swift, certain, and proportionately as severe as the crime. However, without less coercive social institutions, Lynch \& Sabol (2004) suggest that as imprisonment becomes normalized it becomes less effective. During this period incarceration dramatically increased, rising from 503,600 prisoners in 1980 to just over 771,200 prisoners in 1989 (Carson \& Anderson, 2016).

In 1992, Democrats embraced the fervor of popular punitivism by supporting threestrikes laws (Travis \& Western, 2014). The State of Washington enacted the first Three Strikes Law in 1993. In the 1994 State of the Union address President Clinton endorsed "three-strikes laws" (Clinton, 1994). Also, in 1994, President Clinton signed into law a $\$ 30$ million bill that funded mandated life sentences for third time offenders under "threestrikes" laws and expanded the number of prisons and the size of police forces (Alexander, 2012). Over the next three years 24 states enacted three-strikes laws (Austin, Clark, Hardyman, \& Henry, 1998).

These laws were aimed at violent repeat felony offenders and allocated sentences of life without parole. Evaluations of three-strikes laws vary, yet largely conclude there is only a marginal deterrence effect (Marvel \& moody, 2001; Sorensen \& Stemen, 2002). These laws have had unintended consequences. In an evaluation of state sentencing policies, three strikes laws were found to be most effective in incarcerating drug offenders as opposed to violent offenders who were the primary target (Sorensen \& Stemen, 2002). Three-strikes laws, compounded with previous policy changes, added to the number prisoners incarcerated in the United States.

The Bureau of Justice Statistics estimates approximately 771,200 individuals were incarcerated in 1990 (Cohen, 1991). Just before the end of the decade the estimated 
incarcerated population of the United States was over 1.4 million (Carson \& Anderson, 2016). After the turn of the century and in the decade following, the incarceration population rose above 2 million to a peak of over 2.3 million in 2008, before declining slightly to 2.1 million in 2015 (Carson \& Anderson, 2016). The era of mass incarceration gave rise to calls for reform in the early 2000s.

\section{Justice Reinvestment}

The concept of Justice Reinvestment was first introduced (BJA, 2014) with the November 2003 Open Society Institute publication of the monograph "Justice Reinvestment" by Tucker and Cadora (OSI, 2003). According to the authors, the investment should be distributed to four areas: community development, education, health, and rehabilitation and reentry services (Tucker \& Cadora, 2003). Tucker and Cadora (2003), suggest that by investing in the communities that disproportionately feed into the criminal justice system, recidivism can be decreased directly and indirectly by providing rehabilitative and reentry support to the offender. Research on the predictive characteristics of recidivism reveal that offender age, race, ethnicity, prior criminal record (Hepburn and Albonetti, 1994), gender (Gainey et al, 2000), and risk-needs levels (Latessa and Lovins, 2010) all increase the likelihood of recidivating.

Early justice reinvestment implementations had success (BJA, 2014), suggesting it could be a viable method of reduction. The first implementation of justice reinvestment occurred in Connecticut in 2004 (BJA, 2014). Through collaboration with the Counsel of State Governments (CSG) the state created legislation that reinvested approximately $\$ 13$ million in 2005 into reentry programs (CSG, 2017). The goals of the law were to reduce 
the number of people on probation and parole, to create automatic parole hearings after $85 \%$ time served, and to require the implementation of evidence-based reentry programs. The Counsel of State Governments hailed the reforms as the reason Connecticut has one of the fastest decreasing incarcerated populations in the nation (CSG, 2017). In 2007 Texas' Department of Corrections underwent a thorough restructuring, averting approximately $\$ 700$ million in new prison expenses and correctional costs, while reinvesting almost \$250 million into treatment and diversion programs (BJA, 2014; Orrick \& Vieraitis, 2014). Texas achieved these outcomes by redirecting savings to fund evidencebased practices for rehabilitation and support for post-prison release.

In 2010 Congress allocated funding to the BJA to create the Justice Reinvestment Initiative (JRI) to "fund, coordinate, assess, and disseminate state and local justice reinvestment efforts" (BJA, 2014). To date, JRI has provided services and funding to 17 states. The JRI model has seven components: 1) establish a bipartisan working group, 2) analyze and identify cost drivers, 3) negotiate policy options to reduce costs, 4) codify changes, 5) implementation, 6) reinvest, and 7) measure outcomes (BJA, 2014). The broad strokes of the JRI model are intended to allow local and state stakeholders to craft the best fitting solution to each state's needs.

Commonly used benchmarks for justice reinvestment evaluations include populations of incarcerated individuals under correctional control, costs of associated practices, admissions to facilities compared to releases, bed counts, and recidivism rates (Austin et al, 2013; BJA, 2014; Febelo et al, 2007; Bird \& Grattet, 2015). These benchmarks depend on the particular policy that was accepted by each state's legislature (constructed through prioritizing different outcomes as desired by competing interests in 
the political process), and therefore vary between states. Theoretically, differing benchmarks could make state to state comparisons difficult. However, there is much to be gained from comparing different state's attempts in implementing justice reinvestment. While comparing different states in their implementation of justice reinvestment is the direction of future research, I focus here specifically on the state of Oregon. There have been few studies evaluating the aggregate effectiveness of justice reinvestment in each state. These studies have been conducted primarily by the BJA, CSG, and the Vera institute. Most state evaluations report the actual numbers of recidivism and prison populations, and report either a stabilization or decrease of these populations (CSG, 2017; BJA, 2014). The actual data are commonly compared to projections about future behavior of these populations based on multivariate predictions made before the implementation of JRI. While they provide good comparisons, these analyses do not address the actual effectiveness of JRI implementation.

\section{Oregon House Bill 3194}

Between the years 2000 and 2010, Oregon's prison population grew from 9,491 to 13,784 inmates, an increase of nearly $45 \%$ (BJA, 2014). This is roughly the same as the national increase in prison population which increased from 1.4 million to 2.2 million (Carson \& Anderson, 2016). In July of 2011 the Governor ordered the creation of the Commission on Public Safety, which began the discussion to reform and curb the growing inmate population and expenses attributed to them. In 2012, Oregon joined the Justice Reinvestment Initiative (JRI) through the Bureau of Justice Assistance (BJA, 2014). Working with officials from JRI, the commission performed an extensive review of 
criminal justice policy, practices, and costs. The joint efforts resulted in the introduction of House Bill (HB) 3194 in February of 2013. After extensive hearings, work sessions, and adjustments, the bill passed into law in July 2013.

The primary functions of the bill reduced mandatory minimum sentences for marijuana offenses and for driving with a suspended license, increased transitional leave (prior to release) from 30 to 90 days prior to discharge, and provided a comprehensive definition of recidivism (discussed below). The bill also requires Oregon's counties to report their progress and financial spending for community supervision, rehabilitation research, and evidence-based programing to the state's Department of Justice and the Criminal Justice Commission (CJC) for review ( $77^{\text {th }}$ Oregon Legislative Assembly, 2013). After review, counties can apply for the Oregon Justice Reinvestment Grant (OJRG), which the CJC distributes to implement and support the use of evidence-based practices. This is the reinvestment mechanism. Any funds a Department of Corrections (DOC) agency generates at the county level as savings are placed back into the state's general fund, and moneys for the OJRG are then transferred back out of the general fund to the grant, then to individual counties through the CJC.

These revisions are expected to reduce the number of incarcerated individuals in Oregon correctional facilities (Legislative Fiscal Office, 2013). The fiscal impact report from the Legislative Fiscal Office anticipated that the overall bed count in the Oregon Department of Corrections (ODOC) facilities would decrease by 700 beds in the following two years saving approximately $\$ 20$ million. In turn, the legislature's redistribution of funds would increase the aggregate community corrections budget by $\$ 1.3$ million (Legislative Fiscal Office, 2013). Additionally, the bill invests money in a wide variety of 
programs including victim's services, sheriff's departments, and county recidivism reduction efforts.

The legislation provides for flexibility such that each jurisdictional effort to implement JRI may take shape in a variety of ways, as long as it aims to achieve the desired goals of HB 3194. These goals, defined by the bill and the Oregon CJC, are to reduce recidivism, decrease prison usage, and maintain public safety (CJC, 2018). One feature of JRI is the requirement that approximately $3 \%$ of the reinvested funds should go into evaluation research of the specific programs implemented in each county. Subsequently, this has led to a growing number of studies examining specific programs to ensure states are reinvesting into evidence-based practices (BJA, 2014). As large portions of resources are devoted to program fidelity evaluation, few studies have attempted to examine the overall effect of justice reinvestment on criminal justice populations and budgets.

There have been a few comprehensive analyses that have been performed to examine the success of the law's intended effects. The first, a process evaluation published in 2015, states that the prison population has remained relatively flat since justice reinvestment was passed, which has averted operationalizing one constructed facility and the building of another (without the passage of HB 3194 this would have occurred in 2014). This trend was projected to remain below the critical threshold by the end of 2017 (Schmidt \& Officer, 2015). Additionally, short term transitional leave (STTL) usage increased between 2013 and 2015, with almost all counties using STTL (Schmidt \& Officer, 2015). Ultimately, the authors conclude that the continued success of justice reinvestment in Oregon can be best achieved by increased and continued use of the OJR grant and EBPs (Schmidt \& Officer, 2015). 
Furthering the analysis of Oregon's justice reinvestment, the Task Force on Public Safety (TFPS) released an outcome evaluation that states that of all sentencing changes made by HB 3194, STTL was the only change to achieve its projected savings. All other sentencing changes achieved little to no savings (TFPS, 2016). The authors also assess that the utilization of the OJR grant was slow to roll out. This is due to the ground level implementation process of creating local task-forces to address justice reinvestment directives, and the hiring and training of additional officers, providers, and advocates (TFPS, 2016). They note that change in prison use has only just begun in counties that were quick to implement HB 3194 initiatives (TFPS, 2016). Another outcome evaluation was released in 2017. Using cost avoidance methodology, this study shows HB 3194 has avoided almost \$20 million in state DOC costs between 2013 and 2015 (Schmidt, 2017). Furthermore, this is expected to increase to above $\$ 70$ million by 2017 . Local avoidance achieved nearly $\$ 19$ million in the 2013-2015 biennium and $\$ 66.6$ million in the second biennium (2015-2017). The updated 2017-2019 biennium costs suggest savings of nearly $\$ 200$ million of the DOC budget (Schmidt, 2017). In total, the updated estimated net cost avoidance of justice reinvestment between 2013-2019 would be \$254 million (Schmidt, 2017).

This study examines Oregon's success in achieving the goals of justice reinvestment by analyzing the change in justice system populations. No other study has attempted an evaluation of the aggregate effectiveness of JRI reform through quasiexperimental design, thus creating a large gap in understanding the efficacy of justice reinvestment and the Justice Reinvestment Initiative. Thus far, analysis has tended to use prior data to project future expected outcomes. Projections made during the early stages of 
implementation may not reflect current outcome fidelity and effectiveness. This type of reform has been attempted/implemented in seventeen states since 2011. The Bureau of Justice Assistance's 2014 assessment of eight states revealed that all of the states have had a reduction in their prison populations and have had substantial savings in their corrections budgets (BJA, 2014). However, they caution the interpretation of the results because they are made on projected populations before the implementation of the law and state "it would be more accurate to examine specific measures, if possible, about policy use to pinpoint a specific JRI provision's impact" (BJA, 2014; 38). Additionally, there have been no external evaluations (research not conducted by BJA, CSG, Vera) as the time of this writing. This study adds to and addresses this gap in research by evaluating the effectiveness of Oregon's justice reinvestment bill HB 3194 (2013). 
Methods

Time Series in Policy Research

In order to analyze the effect of the HB 3194 (2103), advanced quasi-experimental statistical methods are needed that can account for changes over time. There are multiple ways to address policy effectiveness, including forecasting, ordinary least squares regression analyses (Berry \& Lewis-Beck, 1986), and interrupted time series analysis (ITSA) (Cook \& Campbell, 1979; McDowall et. al, 1980, McCleary et. al, 2017). The interrupted time series quasi-experiment was suggested by Campbell and Stanley (1966) as a form of accounting for historical trends to derive the impact of an intervention on longitudinal data. Time series data are analyzed by the introduction of the intervention, which can produce significant trend or level change (Ramirez and Crano, 2003). To increase validity, an event can be tested by utilizing multiple outcome variables. If the introduction of the independent variable (the intervention) does not produce effects across all dependent variables, the significant changes observed in outcome variables are thought to be associated with a third, unknown factor rather than the intervention (Ramirez and Crano, 2003). This method has been used in the study of interventions across multiple disciplines. Common applications include pharmacology, psychology, public health, and education policy evaluation.

In the field of criminal justice, it can be used for both the evaluation of policy and treatment programs. Jensen and Metsger (1994) employed ITS analysis to review the impact of juvenile waivers to adult court in Idaho. To increase validity, the researchers compared Idaho against Wyoming and Montana (Jensen \& Metsger, 1994). Ramirez and Crano (2003) evaluated California's three-strikes law, spanning the period from 1983 
through 1998. The researchers used two ITSA techniques for three separate analyses. The first assumed an immediate impact model and the second assumed a gradual impact model using the McDowall (1980) methods. The third analysis used the OLS regression technique by Berry and Lewis-Beck (1986). With McDowall impact models they found that the California three-strikes law had no significant impact on all of the dependent variables. The third analysis, proposed by Berry and Lewis-Beck (1986) combines ITSA with OLS regression to prevent the possible inflation of type II error. This analysis concluded that the three-strikes laws did have a significant impact on violent and minor crime rates (Ramirez \& Crano, 2003).

Design

By utilizing interrupted time-series analyses on the bill's intended outcome measures, I evaluate the main research question: Has HB 3194 achieved its goals of decreasing supervision outcomes by reducing incarcerated populations and increasing community supervision populations? According to HB 3194, the Oregon Criminal Justice Commission (CJC) is charged with collecting information and data on, and determining if, counties are implementing evidence-based practices. The CJC utilizes this information to distribute the Oregon Justice Reinvestment Grant (OJRG). This grant is used to initiate and support counties' development of evidence-based practices. This redistribution process is expected to have several impacts, which provide the basis for this study's hypotheses. Hypotheses

On a global scale, HB 3194 intends to shift individuals away from custody institutions to probation or parole supervision. As suggested by the legislation, the count of people in custody supervision should generally be in decline after the implementation 
of HB 3194 (2013). House Bill 3194 was intended to decrease the amount of temporary and emergency beds utilized by all DOC and county facilities (i.e. prisons and jails) by 700 in the $2013-2015$ biennium, and by an additional 840 beds through the $2015-2017$ biennium. The bill's intended outcome is a decrease of roughly 1,500 beds by the end of June 2017 (Legislative Fiscal Office, 2013). Therefore:

$\boldsymbol{H}_{1}-$ After the introduction of $H B$ 3194, prison admissions will decrease.

The release of individuals from custody control to community supervision, where evidence-based practices are required by HB 3194, could result in community supervision populations increasing. More effective programing would have the suggested effect of less recidivism, and therefore more individuals remaining on community supervision. Additionally, justice reinvestment is used to divert offenders to community supervision. One way to capture this is by measuring individuals who receive a presumptive prison sentence but are diverted to probation instead. This is captured by probation admissions. People already in prison or on probation prior to the law's enactment do not fall in the jurisdiction of justice reinvestment or will be picked up later in measurements of recidivism or a future probation admission. This leads to the next two hypotheses:

$\boldsymbol{H}_{2}-$ After the introduction of HB 3194, probation admissions will increase.

$\boldsymbol{H}_{3}-$ After the introduction of $H B$ 3194, the community supervision populations for probation and parole will increase.

Data

Data collected to analyze the effect of HB 3194 (2103) are deidentified monthly aggregated data provided by the senior research analyst at the Oregon Criminal Justice 
Commission. The Oregon CJC has been tasked by the legislature to collect county prison and community supervision data for research, and to ensure the counties are utilizing the funds appropriately. These data sets include: monthly prison admissions for first sentences and parole revocation, monthly probation admissions, and the 2010 and 2013 custody release cohorts. These data sets were selected for the State of Oregon and the three counties in this study.

Because HB 3194 creates overarching changes to the funding and practices of justice agencies, the current study aimed to conduct an overall state evaluation of the outcome measures. A state-wide analysis can provide a general evaluation of the law, but Type II error is at risk because actual results could be over- or under-powered by large counties. For example, Oregon has 36 counties, of which Multnomah County (Portland) contains the largest population: approximately 800,000 people $(200,000$ more than the second largest county, U.S. Census, 2017). A simple state-wide analysis could produce results that were dominated by such a large population center. The reverse is equally possible: if no other county implemented, a simple statewide aggregation of data from a population of about 4,000,000 could indicate no effect despite Multnomah's full implementation of justice reinvestment. Studying all counties for these measures would produce the most accurate depiction of JR in Oregon; however, this is beyond the scope of this study.

This study utilizes three counties as proxies to represent different levels of implementation. As the tables C1 - C3 (appendix) show, the three proxy counties have similar rates for the measures used in this study. The rates were constructed by collecting U.S. Census population estimates for each year (2010 - 2016) for the state and proxy 
counties. Mean values were calculated for the measures in this study by year and divided by the political unit's (i.e. the state of Oregon or counties) estimated population for each year. The low and medium usage counties have similar mean rates of prison admissions, probation admissions, and community supervision populations per year compared to the high usage proxy and the state rates. Therefore, it is important to understand the effects of the law on counties that have medium and low usage of the OJR grant.

Furthermore, person and property crime rates have been generally in decline over the last 20 years but have been slowly increasing since 2010 (CJC Interactive Data, 2018). The high usage proxy's crime rates have been increasing since 2010 as well. In the medium and low usage proxies, behavior, person, and property crime rates have been declining since 2011 (CJC Interactive Data, 2018). Also over this period, the population of Oregon has increased by $6 \%$. The population in the high usage county has increased by $8 \%$ between 2010 and 2016. The medium usage county's population increased by $15 \%$ and the low usage county's population increased by $2 \%$.

As individual counties could have created or funded their own programs that are evidence-based prior to or after the introduction of HB 3194, a proxy measure was created to rank county adoption. This measure includes a count per county of the amount of programming subtypes for which the county had received funds as of October 2017, as a representation for the use of the justice reinvestment grant and implementation commitment. The CJC has divided the areas of funding into seven categories, containing 20 subtypes. The seven categories are: supervision and sanctions, treatment, transition services, victim services, offender services, program management and support, jail pretrial release, and restitution (OCJC, 2017). For example, the offender services category 
includes the subtypes: education, employment, parenting, mentoring, and outreach (OCJC, 2017). One county represents the largest use of reinvestment funds (high usage), one represents moderate use of funds (moderate usage), and one represents the least amount of funding usage (low usage). The high usage county proxy receives funding for 12 programing subtypes; the medium usage county currently receives funds for seven subtypes; and the low usage county currently receives funding for two subtypes (OCJC, 2017). Additionally, counties were selected to have proportional rankings of population within the category of subtypes that were used to select the low, medium and high proxies.

The selection of the three counties based on grant usage could be unintentionally influenced by population. If county usage of the OJR grant is based on population size (which can influence crime rates) then usage of the grant then becomes a proxy for population. However, there is variance in the population growth and crime rates in each political unit and there are similar rates of measures utilized in this study. As table A1 shows, population does not determine the number of subtypes a for which a county will receive funding. Therefore, creating proxies by subtype use provides an accurate measure of justice reinvestment implementation. Additionally, the low usage county was allocated funding for two program subtypes in the 2015-2017 biennium but was awarded funding for 7 programming subtypes in the 2017-2019 biennium (accommodating for changes between bienniums is outside the range of this study).

For the state and selected counties, this design measures the dependent variables before the intervention of HB 3194 and the effects of the intervention on the outcome measures afterwards. Three years of pre-intervention (June 2010 - June 2013) data and three years of post-intervention (July 2013 - July 2016) data were analyzed to reveal the 
changes to community correctional populations and prison admissions. Each of these dependent variables require their own analysis because they are outcome goals of the intervention of HB 3194 (2013) and multiple outcome variables cannot be tested simultaneously.

\section{Dependent Variables}

Dependent variables in interrupted time series analyses are the measure for which the intervention may have an effect. Each of the measures are used as proxy variables that have been used in prior research either together or individually to assess treatment programs. Rather than measure the aggregated totals of prison populations, I study admissions to isolate the effect of the law's implementation. The total number of individuals admitted to prison are analyzed by aggregating all admissions types. Using count data for this study actively seeks to measure that actual change in each political unit. The language of HB 3194 directly calls for counts to be measured in regards to beds and prison populations. Additionally, this study is not attempting to compare counties to one another, to which rates would be essential. This is the direction of future research but is beyond the scope of this study.

Prison Admissions ( $\left.\mathbf{H}_{1}\right)$. Admissions data are used to isolate the effect of the law's effect on the prison population. The total number of individuals admitted to prison per month, prison admissions, is comprised of first sentences and revocations and include all the subcategories that are codified under Oregon law. These subcategories are: property, driving, drug possession, drug, person, sex, and other. 
Property crimes. This refers to all individuals convicted for crimes listed in ORS 137.717: attempt or actual theft (1st and 2nd degree), aggravated theft (1st degree), unauthorized use of a vehicle, mail theft, burglary (1st and 2nd degree), criminal mischief (1st and 2nd degree), computer crime, forgery (1st and 2nd degree), criminal possession of a forged instrument (1st and 2nd degree), fraudulent use of a credit card, identity theft, possession of a stolen vehicle, and trafficking in stolen vehicles.

Driving. Defined under ORS 811.182 as: a suspensions defined under ORS 809.411 (suspension for conviction of crime), ORS 809.409 (revocation for conviction of crime), ORS 813.410 (suspension upon receipt of police report on implied consent test): 0.08 percent or more by weight if the person was not driving a commercial motor vehicle; 0.04 percent or more by weight if the person was driving a commercial motor vehicle; or any amount if the person was under 21 years of age, a suspension of commercial driving privileges under, a suspension of commercial driving privileges under ORS 809.510 (conviction of crime), a suspension of commercial driving privileges under ORS 809.520 (lifetime suspension of commercial driving privileges), a revocation resulting from habitual offender status under ORS 809.640 (procedures on habitual offender determination), a suspension resulting from any crime punishable as a felony, a suspension for failure to perform the duties of a driver under ORS 811.705 (failure to perform duties of driver to injured persons), a suspension for reckless driving under ORS 811.140 (reckless driving), a suspension for fleeing or attempting to elude a police officer under ORS 811.540 (fleeing or attempting to elude police officer), a suspension or revocation resulting from misdemeanor driving while under the influence of intoxicants under ORS 813.010 (driving under the influence of intoxicants). 
Drug possession. Defined between ORS 475.752 to 475.935 and includes possession of all controlled narcotics.

Drug. Defined as manufacturing of all controlled narcotics between 475.752 to 475.935 .

Person. Identified under Oregon law as assault, homicide, robbery, and kidnapping (except robbery 3 ORS 164.395 which is under property).

Sex. Defined as all crimes included in the categories of: rape, sodomy, and sex abuse and other sex crime.

Other. This is a net variable that includes all other crimes; includes failure to appear, failure to register as a sex offender, weapon use, felon with a weapon, and all other crimes not classified in categories above.

Probation Admissions ( $\left.\mathbf{H}_{2}\right)$. The total number of individuals admitted to probation per month is also comprised of the subcategories encompassing all violations of Oregon law. These subcategories are identical to the prison admissions subcategories.

Community Supervision $\left(\mathbf{H}_{3}\right)$. The community supervision populations variable has been constructed by aggregating probation and parole populations by release date by month. The data span from July 2010 to December 2015 as recidivism and release data is collected by cohort (2010 and 2013 cohorts).

The Independent Variable

HB 3194. The primary independent variable in interrupted time series analyses is the introduction of an intervention. For this study, the date House Bill 3194 (2013) was enrolled, July 2013, is the intervention. Enrollment is the date on which the law was passed by both houses. This is separate from roll out, which is the process of implementation. 
There are several ways to create the dummy variable describing the impact of the intervention, for ITS analysis. McDowall (1980) describes four ways an intervention can manifest as a time series: abrupt and permanent effect, gradual and permanent effect, abrupt and temporary effect, or gradual and temporary effect. Of these, three are not appropriate for this study. It is unlikely the bill had an immediate impact on the date of implementation (July 25th, 2013), which excludes both abrupt and permanent, and abrupt and temporary models (McDowall, 1980). This lag is due to the time required to evaluate programs, time needed by CJC to create the infrastructure to distribute funds, and the time needed to implement evidence-based practices in new locations. CJC needing time to create the infrastructure to distribute funds, and the implementation of evidence-based practices in new locations.

The gradual and temporary style of impact, one that increases slowly then decreases, is unlikely because the bill has not been repealed and does not have an automatic repeal measure contained within it. Additionally, as this bill received bi-partisan support to keep the state from having to open another prison, county level buy-in is expected to not decrease. Furthermore, because the state is providing funds to counties for adoption there is an incentive to continue to utilize the components of HB 3194. Therefore, gradual and permanent impact intervention on the dependent variables is suggested to be the most accurate type of impact justice reinvestment will have on the dependent measures. The dependent time series might exhibit a minor abrupt change due to the restructuring of the classification of crimes, but the long-term effect of justice reinvestment is the funding of evidence-based practices. This suggests a gradual change in the dependent measures (McDowall, 1980). 
Analytical Plan

Interrupted time series. According to McDowall, time series data are often skewed and non-normal; they originate from random shocks of data with three types of noise: trend and drift, seasonality, and random error (McDowall, 1980). Trend, drift, and seasonality are non-stationary processes. Trend is the general movement of the data away from its starting point throughout time and drift is movement of the time series in large swings about the starting point (McCleary, 2017). Seasonal data is the periodic, or regularly repeated, variation in the data over time (McDowall, 1980). Traditional interrupted time series analysis requires the time series to be stationary. Stationary time series are fully determined by their mean and autocovariance (McCleary, 2017). The ARIMA function can be used to remove noise due to error and non-stationarity.

Developing the noise component for the ITSA technique is an iterative process that begins with data evaluation. The time series is evaluated by examining the autocorrelation function (ACF) and the partial autocorrelation function (PACF) (McDowall, 1980). The ACF tests for autocorrelation in an autoregressive process, the PACF tests for autocorrelation in the moving average process (McDowall, 1980). Autoregression is serial correlation of one observation with all previous observations (Cook \& Campbell, 1979). Moving average samples have a direct correlation with the immediately preceding observation (Cook \& Campbell, 1979). Both AR and MA time series are stationary, as is white noise (McCleary, 2017). Having autocorrelation in the time series can inflate $t$ statistics (McDowall, 1980), therefore removing any autocorrelation is crucial. 
The goal of an interrupted time series analysis is to develop a model consisting of a linear combination of residual noise and a description of the impact due to the intervention. The complete intervention-impact model is represented by:

$$
Y_{t}=f\left(I_{t}\right)+N_{t}
$$

where $Y_{t}$ is a realization of the time series, $N_{t}$ is the Gaussian distributed stochastic noise and $\mathrm{f}\left(\mathrm{I}_{\mathrm{t}}\right)$ is the transfer function describing the impact of the intervention.

Interrupted time series analysis utilizes a two-step statistical procedure to identify the noise component: ACFs and PACFs are computed for random shocks of real world observations (such as numerical counts of inmates per month in a prison). These allow the analyst to estimate the order of ARIMA function to remove noise. The autoregressive integrated moving average (ARIMA) function produces the stochastic component $\mathrm{N}_{\mathrm{t}}$ (McDowall, 1980). ACFs and PACFs are then computed for this component. If the results are a good fit, the stochastic component $\mathrm{N}_{\mathrm{t}}$ is ready for modeling. If not, another pass through the ARIMA function is required. This process is repeated until the residuals have a normal distribution with zero mean and finite variance (Shumway, 2009; McCleary, 2017).

The ARIMA process has three structural parameters: $p, d$, and $q$, expressed as $(p, d, q)$. The parameter $p$ represents the order of the autoregressive relationship, $\phi$, which can be expressed as:

$$
N_{t}=\sum_{i=0}^{\infty} \phi_{1}^{i} a_{t-i}
$$

where $\phi_{1}$ is the weight attributed to a random shock, a(t), over time. The time series observations consist of the sum of the shocks, weighted in fractions and degraded over time 
until the end of the series. Effectively this means that for any given observation, the observation immediately preceding it has the most impact on the current observation, and the farthest observation has the least impact (Campbell \& Stanley, 1963). For example, if $\phi=.5$, the value of the most recent prior sample is halved, but the sample prior to that is quartered. This ARIMA process is expressed as ARIMA $(p, 0,0)$. The purpose of the $(p, 0,0)$ model is to remove the autoregressive relationship from the time series. Ultimately, this filtering process removes the influence of all previous observations from the current observation.

Trend is the movement of the observations in one direction, either up or down. With drift, the observations "walk" both up and down over time (McDowall, 1980, McCleary, 2017). The structural parameter $d$ addresses the non-stationary components, trend and drift, in the data by differencing the time series. Parameter $d$ indicates the order of lag between observations. In a given time-series, the first shock is the sum of all historical shocks not accounted for in the data. A random shock enters a filter where a constant is added and it is integrated (summed) with all previous shocks (Campbell \& Stanley, 1963). This can be expressed as:

$$
N_{t}=\sum_{i=0}^{\infty} a_{t-i}
$$

where $a_{t}$ are successive random shocks and $\mathrm{N}_{t}$ is a realization of the sum of the previous shock subtracted from the current shock for each time step. Differencing is performed by subtracting the first observation from the second, the second from the third, and so on. This is expressed as ARIMA $(0, d, 0)$. This form of filtering makes the data stable so it can be 
analyzed through its residuals. However, because the regression model (discussed below) uses trend to estimate parameters, differencing will not be utilized.

The structural parameter $q$ represents the serial dependency of a moving average relationship (McDowall et. al, 1980). In this case the preceding shock is used to predict the current observation:

$$
N_{t}=a_{t}-\theta_{1} a_{t-1}
$$

where $\theta$ is constrained by the bounds of invertibility $(-1<\theta<+1)$, expressed as ARIMA $(0,0, q)$ (McDowall, 1980, McCleary, 2017). Filtering the moving average from data removes the influence of the previous observation from the current observation.

ARIMA processes may be conceptualized as raw data passing through a series of filters in order to produce a time series observation (Campbell \& Stanley, 1963). Additionally, ARIMA models can also control for seasonality in the data, where the data behaves cyclically. The seasonality process is relatively the same as $p, d$, and $q$ models. For example, given a seasonal moving average model of monthly data, where the current month observation is related to the month in the previous year. Then:

$$
N_{\mathrm{t}}=a_{t}-\theta_{12} a_{t-12}
$$

where we account for a lagged relationship of 12 months. These models are expressed as ARIMA $(p, d, q)(P, D, Q)_{\mathrm{s}}$ (McDowall, 1980; McCleary, 2017). For these processes, the lowercase parameters indicate sequential values of autocorrelation or differencing and the uppercase values indicate seasonality followed by the $s$ value which indicates the repeat period.

Once the noise model, $\mathrm{N}_{\mathrm{t}}$, has been adequately described it is combined with the intervention-impact transfer function to evaluate the efficacy of the intervention. The 
intervention component $\mathrm{I}_{\mathrm{t}}$, a dummy variable (frequently a step function), is used to assess impact (Campbell \& Stanley, 1963). The transfer function for the gradual and permanent impact model can be written as:

$$
\mathrm{f}\left(\mathrm{I}_{\mathrm{t}}\right)=\left(\delta \mathrm{y}_{\mathrm{t}-1}+\omega \mathrm{I}_{\mathrm{t}}\right)
$$

where $\delta$ is a scalar effecting the trend and $\omega$ is the correlation coefficient describing the change in mean. Plugging the transfer function into equation (1.1) yields the gradual and permanent model:

$$
\mathrm{Y}_{\mathrm{t}}=\left(\delta \mathrm{y}_{\mathrm{t}-1}+\omega \mathrm{I}_{\mathrm{t}}\right)+\mathrm{N}_{\mathrm{t}}
$$

where $\mathrm{N}_{\mathrm{t}}$ is the ARIMA $(\mathrm{p}, \mathrm{d}, \mathrm{q})(\mathrm{P}, \mathrm{D}, \mathrm{Q})$ model, and the transfer function for $\mathrm{I}_{\mathrm{t}}(0$ or 1$)$ represents the presence of the intervention component. Prior to the intervention, $I_{t}=0$, and after the intervention, $I_{t}=1$. The intervention component is then combined with impact patterns to produce the assessment. For the gradual permanent impact pattern described in equation 1.6, $\omega$ represents the estimate of the difference between pre-level and post-level, and $\delta$ represents an estimate of the change in trend.

I use generalized least squares regression analysis to determine the coefficients for changes in trend and mean to model the impact of the intervention on the post time series (Berry and Lewis-Beck, 1986). Regression analysis has been used in many interrupted time series studies (e.g. Sundt et. al, 2016; Ramirez \& Crano, 2003; Jensen \& Metsger, 1994; Simonton, 1977).

The Berry and Lewis-Beck method of analysis requires several assumptions. These assumptions include no specification error, absence of measurement error, absence of perfect multicollinearity of independent variables, homoscedasticity, absence of autocorrelation, and that data are normally distributed (Berry and Lewis-Beck, 1989; 220). 
Specification error occurs when both the independent variable is nonlinearly related to the dependent variable and irrelevant independent variables are included in the model (Berry \& Lewis-Beck, 1989). The independent variables in the regression model for this study are time, impact and trend. Time is a sequence counter beginning with 1 at the first time point and increasing by 1 at each step to the last observation. Impact, coded with the variable name Ilevel, represents $\mathrm{I}_{\mathrm{t}}$ which has the value zero before the intervention and 1 at the intervention and thereafter for each time point. Trend is a sequence counter beginning with 1 at the intervention and increasing by 1 at each time step to the last observation, and 0 before the intervention. The model variables of time, impact, and trend are all linearly related and relevant independent variables; therefore, the assumption of no specification error is met.

Multicollinearity is the inclusion of two or more variables in a model that are highly correlated. In interrupted time series, this is an issue within multiple ITS, as an event may occur at the same time as another event that may affect the dependent variable (Berry \& Lewis-Beck, 1989). This assumption is met because the data are not affected by any other major changes to Oregon's criminal justice system (through law) in the 2013 legislative session, and therefore no other event will be included in the model. No homoscedasticity is a condition such that all vectors have the same finite variance (Field, 2013). As there are no other independent variables included in this analysis, homoscedasticity is not an issue. Normality tests were performed on all measures and are included in appendix B. To correct non-normal data, filtering the data through AR and MA processes makes the data normally distributed. Autocorrelation is the greatest threat to regression modeling with time series data because it invalidates the coefficient significance tests and can inflate the t-ratio 300- 
400\% (McDowall, 1980: 13). Autocorrelation is removed from the model by using AR or MA processes in the GLS regression.

The method of model evaluation and fit is an iterative one. First, identification (based on ACF and PACF) guides model selection. The parameters $\theta$ (AR) and $\phi$ (MA) are estimated through the ARIMA process, and if the estimations are not statistically significant the researcher must select a new model (identification) (McDowall, 1980). Additionally, the model can be assessed by evaluating Q after applying the Ljung-Box test (McCleary, 2017). Q statistics that fail to reach significance at $p<.05$ reject the null hypothesis that the residuals of the ARIMA process are not different from white noise and the model is therefore acceptable (McCleary, 2017). If the conditions are not met, one must return to identification. Once these conditions are met the ARIMA analysis is appropriate and the model is accepted. For the ITSA method (Cook \& Campbell, 1979; McDowall et. al, 1980, and McCleary et. al, 2017) all parameters $(\theta, \phi, \delta, \omega)$ must obtain significance for the intervention to have had an effect on the outcome measures. Here, I use the ARIMA process to estimate autocorrelation and to determine the $p$ and $q$ parameters for the GLS regression model. The generalized least squares (GLS) regression is fit by maximum likelihood estimation with ARMA parameters determined by the previous ARIMA model. GLS regression accounts for correlation terms inside the model (Fox \& Weisberg, 2010) as opposed to performing an OLS regression and manually calculating new coefficients with the AR and MA parameters (Lewis-Beck, 1980). The formula for the single intervention regression is:

$$
Y_{t}=b_{0}+b_{1} X_{1 t}+b_{2} X_{2 t}+b_{3} X_{3 t}
$$


where $Y_{t}=$ the individual time series observations of the dependent variable, $X_{i t}=$ time, the counter from 1 to the end of the time series, $X_{2 t}=$ Level, the dummy variable signifying the presence of the intervention, and $\mathrm{X}_{3 \mathrm{t}}=$ trend, the time counter beginning at the point the intervention has started (Lewis-Beck, 1980). A counterfactual can be calculated from the intercept $\left(b_{0}\right)$ and the slope of the pre-intervention period $\left(b_{1}\right)$ and projected into the post intervention period. This models what the data might look like if the intervention had not occurred. By taking the last value of the counterfactual and the last value of the fitted data, the absolute and relative change can be calculated. These formulas are:

$$
\begin{aligned}
& \text { Absolute }=f i t_{t N}-c f a c_{t N} \\
& \text { Relative }=\frac{f i t_{t N}-c f a c_{t N}}{c f a c_{t N}} * 100
\end{aligned}
$$

If the absolute and relative change are positive, there has been an increase in that population. If they are negative, there has been a decrease. The result of this analysis is compared to the projected outcomes hypothesized by the enrolled bill and the Justice Reinvestment Initiative for the effectiveness of the legislation. The estimated total change is calculated as:

$$
\text { Estimated Total Change }=\sum_{t=1}^{n} F i t_{t}-\sum_{t=1}^{n} C f a c_{t}
$$

where $t=$ the first month in the post period, $\mathrm{n}=$ the last month in the post period, Fit $\mathrm{t}=$ the trend fitted to the post-data from the GLS regression model, and $\mathrm{Cfac}_{\mathrm{t}}=$ the projection of the pre-data trend. The estimated total change is calculated by first summing the change in trend per month for the counterfactual post period, then summing the change in trend per month for the impacted post intervention period (the fitted values), then differencing the counterfactual from the fitted. 
Table 1. State of Oregon Descriptive Statistics by Year

\begin{tabular}{|c|c|c|c|c|c|}
\hline $\begin{array}{l}\text { Prison } \\
\text { Admissions }\end{array}$ & $\mathrm{N}$ & Std. Deviation & Mean & $\%$ Change & $\begin{array}{l}\text { Mean Rate } \\
\text { Per } 100,000\end{array}$ \\
\hline 2010 & 7 & 25.409 & 389.43 & & 10.15 \\
\hline 2011 & 12 & 32.879 & 387.83 & $-0.41 \%$ & 10.03 \\
\hline 2012 & 12 & 33.571 & 397.92 & $2.60 \%$ & 10.22 \\
\hline 2013 & 12 & 27.767 & 412.42 & $3.64 \%$ & 10.52 \\
\hline 2014 & 12 & 36.581 & 396.25 & $-3.92 \%$ & 10.00 \\
\hline 2015 & 12 & 31.644 & 405.08 & $2.23 \%$ & 10.09 \\
\hline 2016 & 7 & 39.245 & 387.86 & $-4.25 \%$ & 9.49 \\
\hline $\begin{array}{l}\text { Probation } \\
\text { Admissions }\end{array}$ & $\mathrm{N}$ & Std. Deviation & Mean & $\%$ Change & $\begin{array}{l}\text { Mean Rate } \\
\text { Per } 100,000\end{array}$ \\
\hline 2010 & 7 & 28.652 & 894.43 & & 23.31 \\
\hline 2011 & 12 & 50.389 & 902.83 & $0.94 \%$ & 23.35 \\
\hline 2012 & 12 & 86.473 & 909.67 & $0.76 \%$ & 23.36 \\
\hline 2013 & 12 & 49.001 & 901.83 & $-0.86 \%$ & 23.01 \\
\hline 2014 & 12 & 52.762 & 964.17 & $6.91 \%$ & 24.34 \\
\hline 2015 & 12 & 38.924 & 919.25 & $-4.66 \%$ & 22.89 \\
\hline 2016 & 7 & 54.381 & 911.00 & $-0.90 \%$ & 22.30 \\
\hline $\begin{array}{l}\text { Community } \\
\text { Supervision }\end{array}$ & $\mathrm{N}$ & Std. Deviation & Mean & $\%$ Change & $\begin{array}{l}\text { Mean Rate } \\
\text { Per } 100,000\end{array}$ \\
\hline 2010 & 6 & 31.552 & 1164.50 & & 30.35 \\
\hline 2011 & 12 & 61.926 & 1162.58 & $-0.16 \%$ & 30.07 \\
\hline 2012 & 12 & 92.131 & 1176.50 & $1.20 \%$ & 30.21 \\
\hline 2013 & 12 & 65.643 & 1164.67 & $-1.01 \%$ & 29.71 \\
\hline 2014 & 12 & 73.494 & 1214.92 & $4.31 \%$ & 30.67 \\
\hline 2015 & 12 & 61.045 & 1175.00 & $-3.29 \%$ & 29.25 \\
\hline
\end{tabular}

Table 1.A Yearly Mean Rates by Measure and Political Unit (Descriptive Statistics Tables)

\begin{tabular}{lcccc}
\hline \multicolumn{5}{c}{ Prison Admission } \\
\hline Year & State & High Usage & Med Usage & Low Usage \\
\hline 2010 & 10.15 & 10.29 & 10.23 & 10.24 \\
2011 & 10.03 & 10.46 & 8.81 & 4.10 \\
2012 & 10.22 & 11.08 & 9.53 & 8.99 \\
2013 & 10.52 & 11.19 & 8.89 & 9.35 \\
2014 & 10.00 & 9.32 & 10.48 & 7.51 \\
2015 & 10.09 & 8.82 & 9.86 & 10.81 \\
2016 & 9.49 & 8.88 & 9.93 & 9.42 \\
& & & & \\
Year & & Probation Admission & \\
\hline 2010 & 23.31 & 20.75 & 31.59 & 32.59 \\
2011 & 23.35 & 21.23 & 25.19 & 33.05 \\
2012 & 23.36 & 20.88 & 24.31 & 25.50 \\
2013 & 23.01 & 21.60 & 25.02 & 30.71 \\
2014 & 24.34 & 21.13 & 28.60 & 41.96 \\
2015 & 22.89 & 17.52 & 25.82 & 30.90 \\
2016 & 22.30 & 17.74 & 22.93 & 31.44
\end{tabular}

\begin{tabular}{ccccc} 
Year & \multicolumn{4}{c}{ Community Supervision } \\
\hline 2010 & 30.35 & 31.98 & 33.90 & 34.29 \\
2011 & 30.07 & 32.50 & 28.32 & 28.95 \\
2012 & 30.21 & 30.59 & 25.86 & 30.00 \\
2013 & 29.71 & 30.53 & 27.84 & 33.36 \\
2014 & 30.67 & 30.54 & 31.63 & 36.74 \\
2015 & 29.25 & 26.36 & 27.01 & 32.37 \\
\hline \multicolumn{5}{l}{ Note: Population data was collected from the U.S. Census }
\end{tabular}
(U.S. Census, 2018)

\section{Analytical Tools}

I used the $\mathrm{R}$ statistical platform and the R programming language (R Core Team, 2017) for all analyses in this study. Functions contained in the base package of $\mathrm{R}$ that are used for analyses are: $I m$, used to model initial OLS regression statistics for the raw data; arima, used to perform ARIMA filtering; box.test, which calculates the Q statistics; acf is used to create ACF and PACF plots; and plot, which is used to create all other plots. Additional packages used include lmtest, which contains coefficient testing for the regression analyses (Zeileis \& Hothorn, 2002), and nlme (linear and nonlinear mixed effects models) for performing the GLS regression with ARMA parameters (Pinheiro et. al., 2018). 


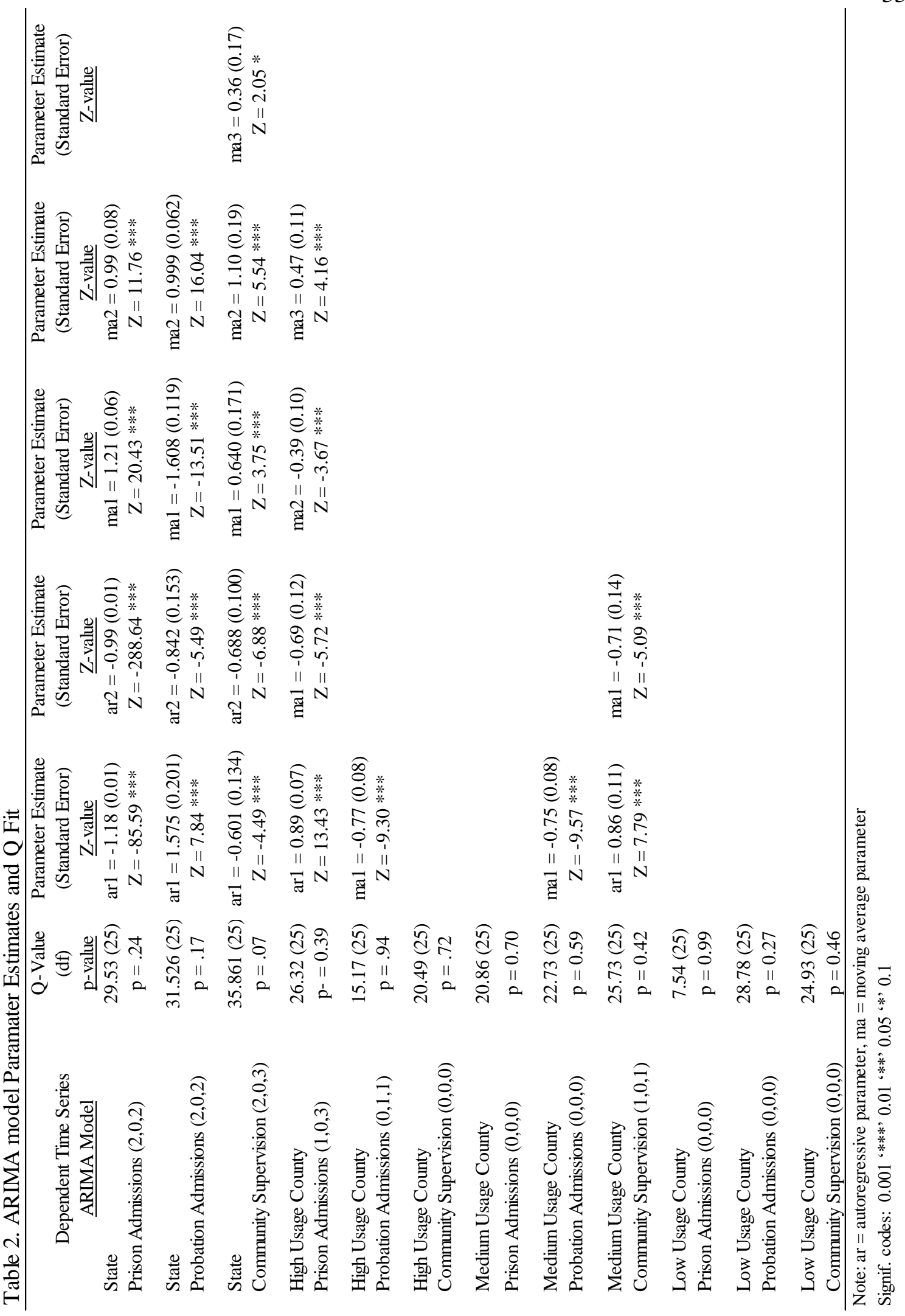


Results

The high usage county utilizes the OJR grant for 12 program subtypes. The high usage proxy also processes the most individuals of the counties in this study (see Table C1). The medium usage county utilizes the OJR grant for seven program subtypes and has jurisdiction over smaller criminal justice populations than high usage county (Table C2). The low usage county uses grant funding for two program subtypes and has very small custody and community supervision populations (Table C3) when compared to both the high usage and the medium usage counties. However, because the rates are similar, this suggests that each of the proxy counties is affected by similar prison and probation admissions and individuals on community supervision. Because there is an unequal distribution of programing subtypes receiving funds in the proxy counties, the existence of justice reinvestment might have different outcomes in the three proxy counties.

H1 - After the introduction of HB 3194, prison admissions will decrease.

The plots of ACF and PACF of the raw State of Oregon prison admission data show serial autocorrelation for both the autoregressive and moving average processes. The ARIMA process provided the best fit with an ARIMA $(2,0,2)$ model where all autoregressive and moving average terms are statistically significant (shown in Table 3) and the model residuals were not different than white noise $(p=0.24)$. The GLS regression by maximum likelihood with correlation ARMA $(2,2)$ coefficients are shown in Table 4. This produced an intercept of 381.23 , a positive slope of 0.691 , an immediate decrease at the time of intervention of $1.17(p=0.837)$ that was not significant, and a significant change 


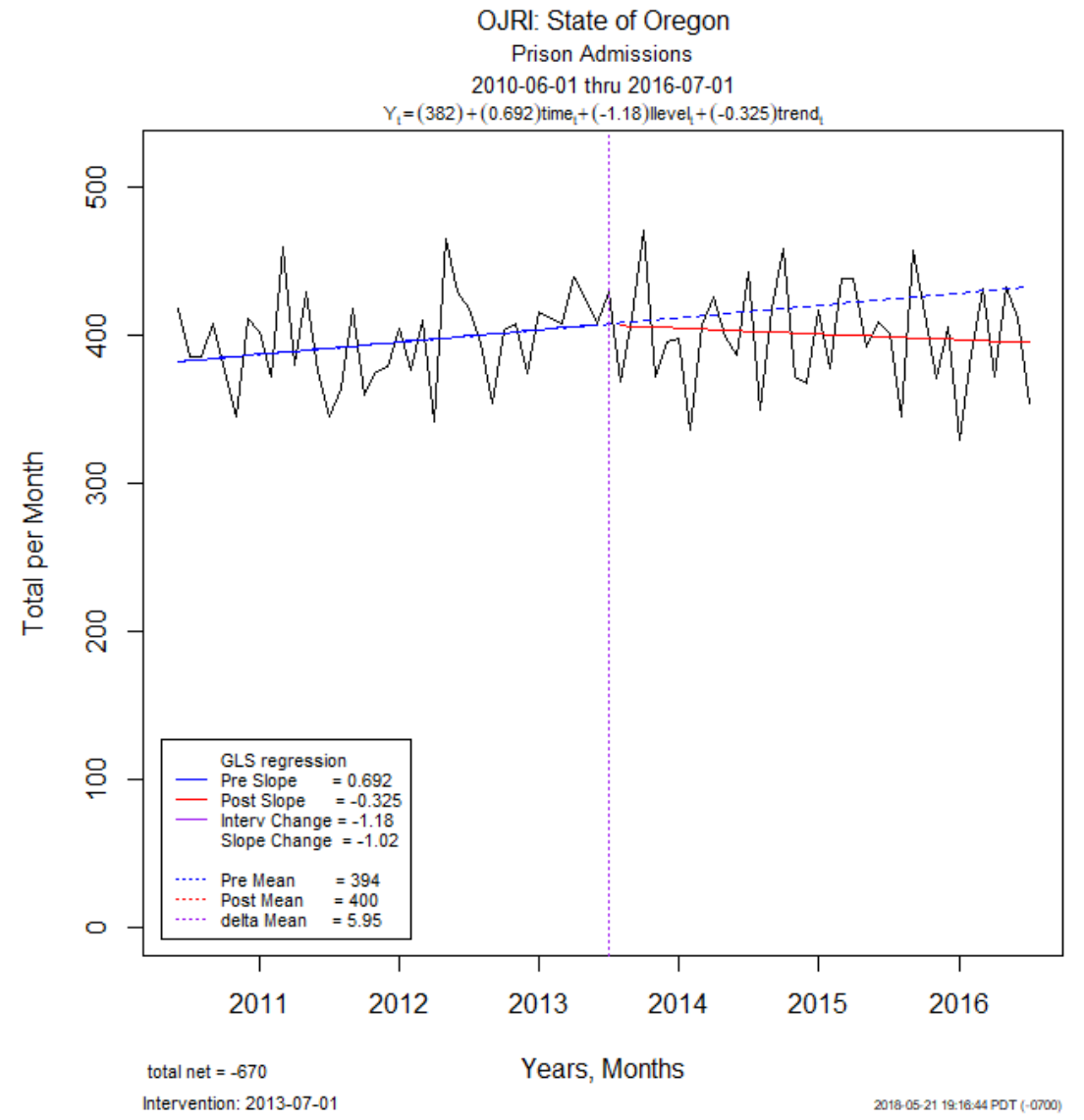

Figure 1. State of Oregon Prison Admissions by Month, June 2010 to July 2016

between the pre-slope and the post-slope of $-1.0169(p<0.001)$ is observed. This results in the post slope being -.325 . The pre-trend indicates increasing prison admissions before the intervention, and after the intervention the count of people being admitted to prison starts to decline. This suggests that HB 3194 has had a significant effect on prison admissions for the state. Modeling the counterfactual (if there had been no intervention), there has been a decrease of 37.8 individuals not being admitted to prison by July 2016 . The estimated total change over the post intervention period is a decrease of 640 admissions to the prison system statewide, supporting the hypothesis.

For the high usage county's prison admission raw data (Figure 2), ACF and PACF showed serial autocorrelation. This was best filtered by an ARIMA $(1,0,3)$ model, where 
Table 3. Hypothesis 1, Regression Coefficients and Absolute \& Relative Change

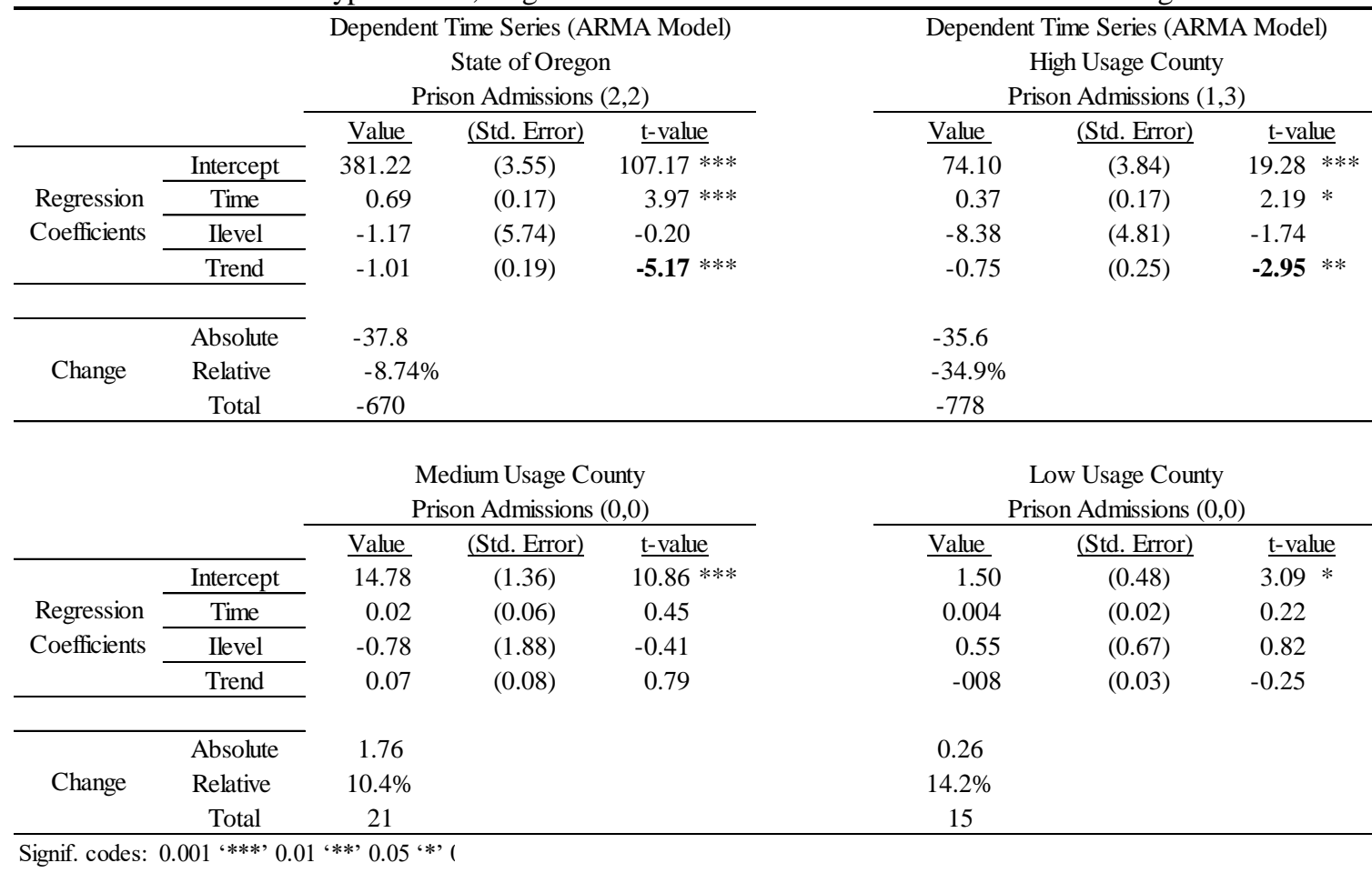

are AR and MA parameters were significant (Table 2). The residuals from the ARIMA $(1,0,3)$ model were not different from white noise $\left(\mathrm{X}^{2}=26.323, \mathrm{df}=25, p=0.390\right)$. Therefore, I conclude this model best filters the raw data. The GLS regression with ARMA $(1,3)$ produced an intercept of 74.103 , a pre-intervention slope of 0.375 , a non-significant immediate change in level at the time of intervention of $-8.380(p=0.086)$, and a significant change in slope of $-0.755(p=0.004)$ which yields a post slope of -0.38 . As can be seen in figure 3 , there is a substantial change in slope between the pre and post periods. Prior to HB 3194 prison admissions were increasing at a rate of .375. After the passage of HB 3194 (2013) there is a decreasing trend. Although there appears to be a drop directly after the bill's enactment, this is not significant. The absolute change at the end of the study period is a decrease in admissions for the high usage county of $35.6(-34.9 \%)$ individuals. Between July 2013 and July 2016, the estimated total change in prison admissions in the high usage 


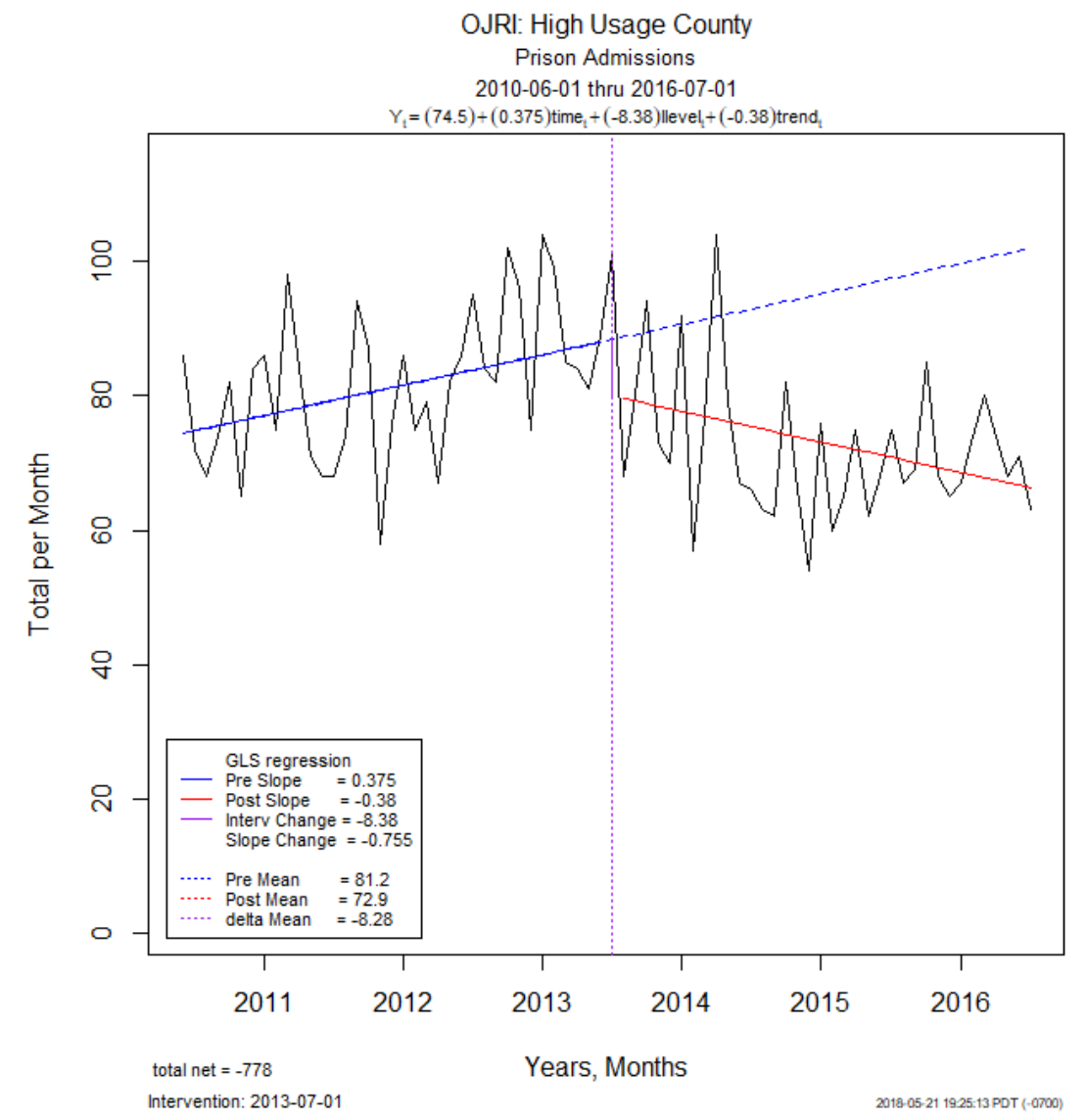

Figure 2. The High Usage County Prison Admissions by Month, June 2010 to July 2016

county was a decrease of 778 individuals, which supports the hypotheses and is in concert with the State result.

The medium usage county prison admissions did not contain autocorrelation. The Box-Ljung test revealed that the residuals from an ARIMA $(0,0,0)$ were not different from white noise $\left(\mathrm{X}^{2}=20.86\right.$, $\left.\mathrm{df}=25, p=0.700\right)$ so the model is accepted. GLS regression without AR or MA parameters coefficients (Table 3) are an intercept of 14.783, a pre-slope of 0.028 , a non-significant immediate change in level of $-0.783(p=0.679)$, and a nonsignificant change in pre-slope $(0.028)$ and post-slope $(0.099)$ of $0.070(p=0.426)$. The regression reveals that prison admissions in the medium usage county were gradually increasing before the intervention, and despite a slight dip in level at the intervention, the 
overall rate of prison admissions stays about the same through July 2016. The absolute change in July 2016 between the fitted values and the projection of the pre-intervention trend is an increase of $1.76(10.4 \%)$ individuals. The estimated total change over the post intervention period is an increase of 21 admissions to prison from the medium usage county. This finding does not support $\mathrm{H}_{1}$.

There were sections of the data missing in the prison admissions data sets of the low usage county. The missing months are assumed to be months were no individuals were admitted to prison in order to maintain the uniform spacing of the time series. Because the base rate of the low usage county is small it is possible that the county saw no prison admissions. However, this could be an absence of reporting; and therefore, filling the missing months with a 0 value would be inaccurate. I acknowledge this as a limitation. No autocorrelation was found when individual ACF and PACF plots were made. All ARIMA $(0,0,0)$ model residuals were no different than white noise (see Table 2$)$. The regression without AR or MA parameters (Table 3) for prison admissions predicted the intercept is 1.500 , the pre-slope is 0.004 , a non-significant change in level of $0.555(p=0.411)$ and a non-significant change from pre $(0.004)$ and post $(-0.004)$ slopes of $-0.008(p=0.798)$. The regression shows that prison admissions in the low usage county stayed relatively the same before and after the effective date of the new law. The predicted absolute change between the counterfactual and fitted values in July 2016 was an insignificant increase of 0.265 (14.2\%) in admissions to prison in the low usage county. During the post-intervention period, the estimated total change is an increase of 15 people admitted to prison, which does not support $\mathrm{H}_{1}$. 
Table 4. Hypothesis 2, Regression Coefficients and Absolute \& Relative Change

\begin{tabular}{|c|c|c|c|c|c|c|c|}
\hline & & \multicolumn{3}{|c|}{$\begin{array}{c}\text { Dependent Time Series (ARMA Model) } \\
\text { State of Oregon } \\
\text { Probation Admissions }(2,2)\end{array}$} & \multicolumn{3}{|c|}{$\begin{array}{c}\text { Dependent Time Series (ARMA Model) } \\
\text { High Usage County } \\
\text { Probation Admissions }(0,1)\end{array}$} \\
\hline & & $\underline{\text { Value }}$ & (Std. Error) & t-value & $\underline{\text { Value }}$ & (Std. Error) & $\underline{\mathrm{t} \text {-value }}$ \\
\hline \multirow{4}{*}{$\begin{array}{l}\text { Regression } \\
\text { Coefficients }\end{array}$} & Intercept & 909.87 & (14.51) & $62.67 * * *$ & 150.48 & $(5.32)$ & $28.27 * * *$ \\
\hline & Time & -0.31 & $(0.72)$ & -0.42 & 0.46 & $(0.24)$ & 1.89 \\
\hline & Ilevel & 50.71 & (23.50) & $2.15 * *$ & 1.77 & (7.35) & 0.24 \\
\hline & Trend & -0.71 & $(0.92)$ & -0.78 & -1.49 & $(0.34)$ & $-4.31 * * *$ \\
\hline \multirow{5}{*}{ Change } & Absolute & 24.8 & & & -52.0 & & \\
\hline & Relative & $2.8 \%$ & & & $-28.2 \%$ & & \\
\hline & Total & 1324 & & & -898 & & \\
\hline & & \multicolumn{3}{|c|}{$\begin{array}{c}\text { Medium Usage County } \\
\text { Probation Admissions }(0,0)\end{array}$} & \multicolumn{3}{|c|}{$\begin{array}{c}\text { Low Usage County } \\
\text { Probation Admissions }(0,0)\end{array}$} \\
\hline & & Value & (Std. Error) & t-value & Value & (Std. Error) & t-value \\
\hline \multirow{4}{*}{$\begin{array}{l}\text { Regression } \\
\text { Coefficients }\end{array}$} & Intercept & $\overline{47.70}$ & $(2.81)$ & $\overline{16.94} * * *$ & $\overline{7.98}$ & $(1.13)$ & $\overline{7.04} *$ \\
\hline & Time & -0.31 & $(0.12)$ & $-2.44 *$ & -0.05 & $(0.05)$ & -1.10 \\
\hline & Ilevel & 11.53 & (3.90) & $2.95 * *$ & 2.11 & (1.57) & 1.34 \\
\hline & Trend & 0.21 & $(0.18)$ & 1.14 & 0.04 & $(0.07)$ & 0.51 \\
\hline \multirow{3}{*}{ Change } & Absolute & 19.0 & & & 3.48 & & \\
\hline & Relative & $78.2 \%$ & & & $93.2 \%$ & & \\
\hline & Total & 531 & & & 97 & & \\
\hline
\end{tabular}

H2 - After the introduction of HB 3194, the probation admissions will increase.

The State of Oregon's probation admissions ACF and PACF plots indicate the data had serial autoregressive and moving average correlation. The data are best represented by an ARIMA $(2,0,2)$ model. All autoregressive and moving average parameters were statistically significant. Additionally, the model residuals were no different than white noise when passed through the Box-Ljung test $\left(\mathrm{X}^{2}=31.526, \mathrm{df}=25, p=0.172\right)$ therefore I conclude that the ARIMA $(2,0,2)$ model is the best fit. The regression accounting for the ARMA $(2,2)$ process coefficients (Table 4) produced an intercept of 909.87, a preintervention slope of -0.3070 , a significant immediate change in level at the intervention point by $50.715(p=0.034)$, and a non-significant change between the pre-slope and postslope of $-0.719(p=0.439)$. The post intervention slope is -1.027 . This suggests that before the intervention, the use of probation admissions was in a slight decline. After the 


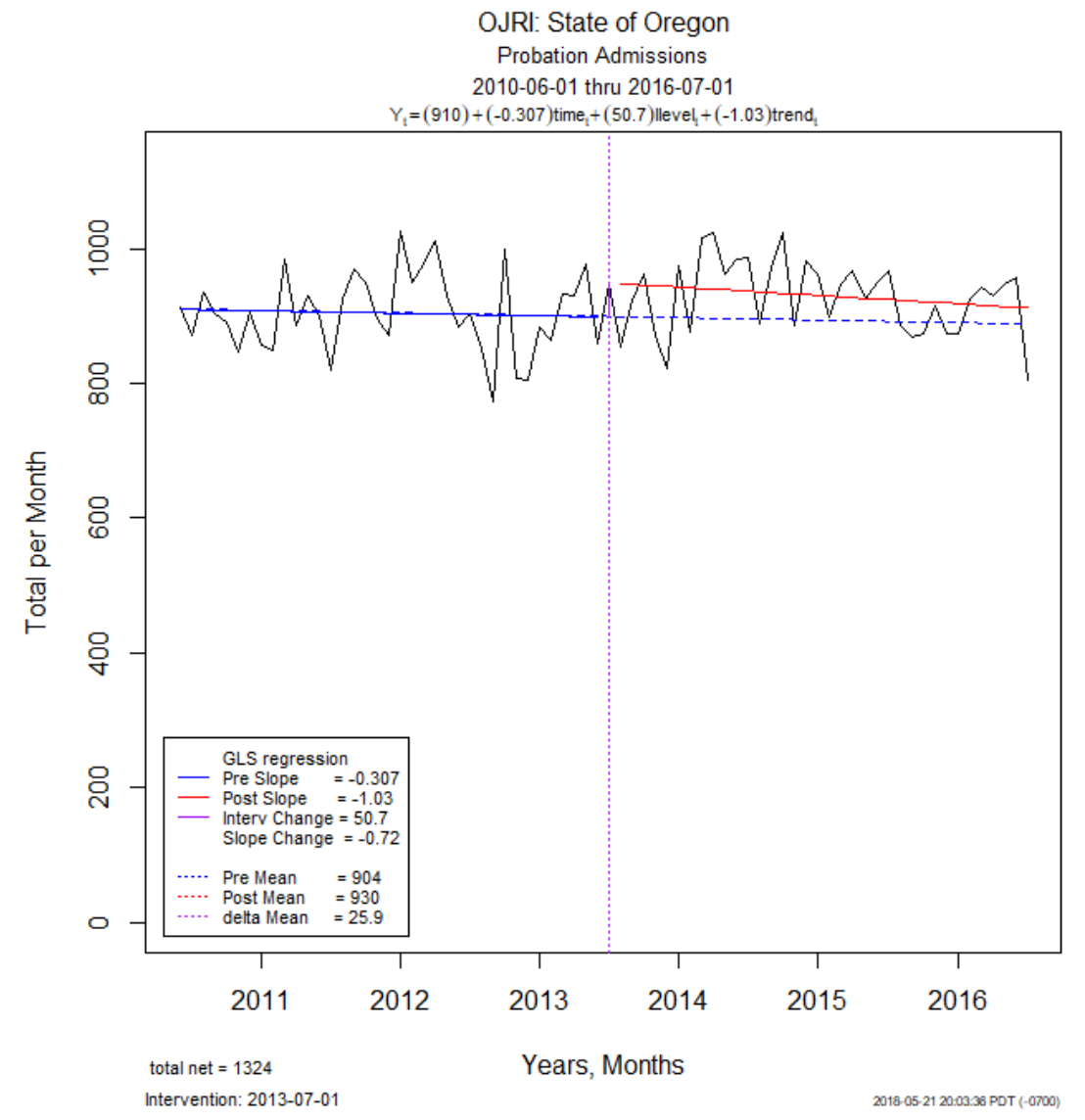

Figure 3. Oregon Probation Admissions by Month, June 2010 to July 2016

intervention, probations admissions increased after the enactment of justice reinvestment, but continued to decline. However, by the end of the study period, more individuals were admitted to probation than if HB 3194 (2013) had not been passed (Figure 3) which does support $\mathrm{H}_{2}$. Calculating the absolute change as of July 2016, there was an increase of 24.8 individuals (2.8\%) from the projected counterfactual. The estimated total change suggests that the State of Oregon had an increase of 1,324 individuals admitted to probation between July 2013 and July 2016.

The high usage county's probation admissions (Figure 4) data showed moving average correlation when ACF and PACF plots were made. The time series is best filtered 


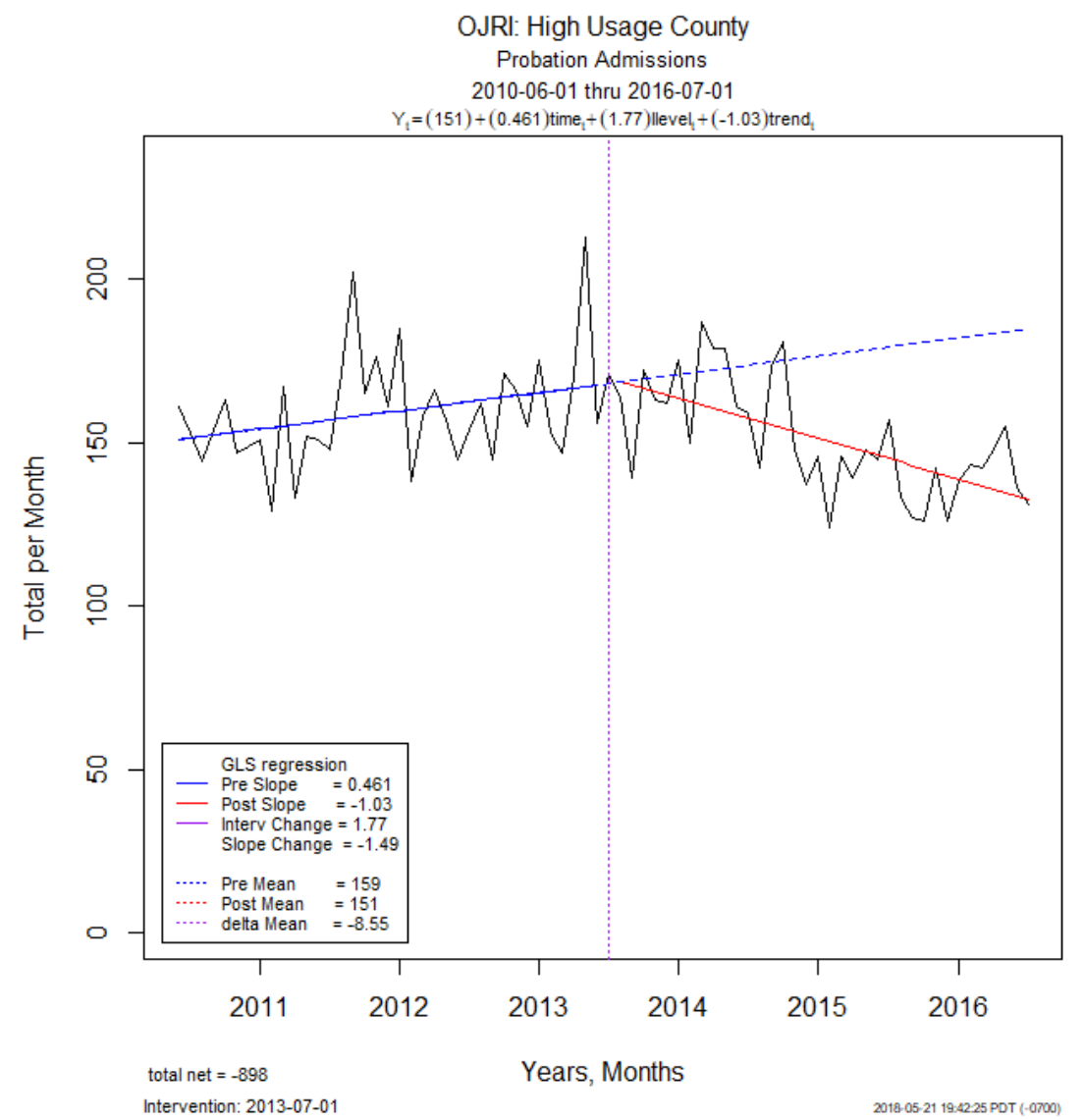

Figure 4. The High Usage County Probation Admissions by Month, June 2010 to July 2016

by an ARIMA $(0,1,1)$ model due to the amount of drift in the series. The MA parameter was significant (Table 4) and the residuals were not different from white noise $\left(\mathrm{X}^{2}=\right.$ 15.166, df $=25, p=0.937$, which provides the best fit model for filtering. The GLS regression with ARMA $(0,1)$ produced an intercept of 150.489 , a pre-intervention slope of 0.461, a non-significant immediate change in level at the time of intervention of 1.768 ( $p$ $=0.810)$, and a significant change between the pre-slope (0.46) and post intervention slope (1.03) fits of $-1.493(p=0.0001)$. These results are shown in Table 4 . The count of prison admissions is increasing before the intervention but is declining after the passage of justice reinvestment with no immediate change at the time of the intervention. The change in slope is in the opposite direction than was expected and does not support $\mathrm{H}_{2}$. Calculating the 


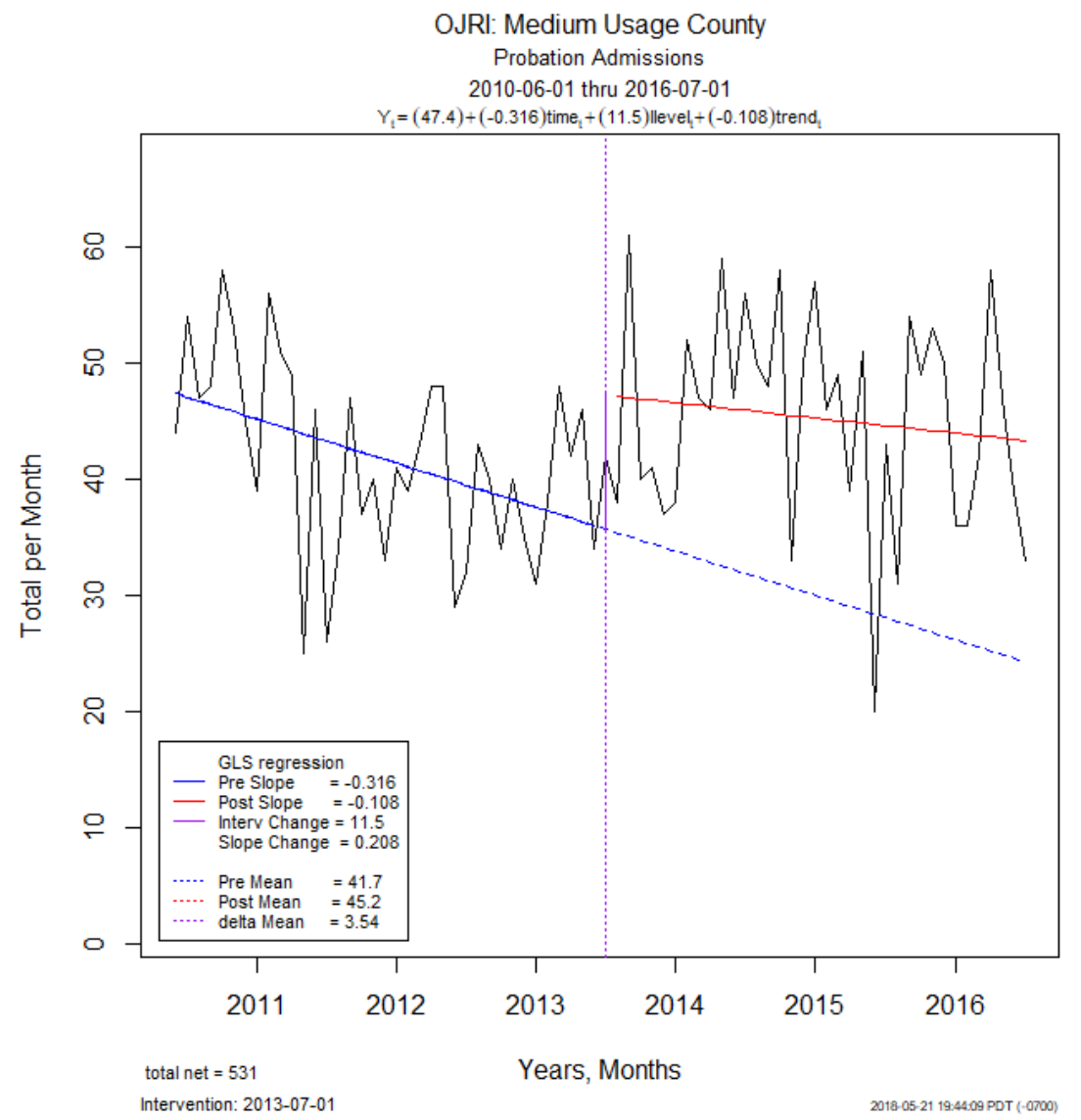

Figure 5. Medium Usage County Probation Admissions by Month, June 2010 to July 2016

absolute change from the end of the counterfactual and the real fitted data produced a decrease of 52 individuals being admitted to probation which is a reduction of $28.2 \%$ by July 2016. After the enrollment of HB 3194, the estimated total change in probation admissions is a decrease of 898 admissions in the high usage county.

Probation admissions in the medium usage county (Figure 5) also did not show autocorrelation of the raw data. The Box-Ljung test supports this assessment as the residuals of the ARIMA $(0,0,0)$ model are the same as white noise $\left(X^{2}=28.006, \mathrm{df}=25\right.$, $p=0.307)$. The model is accepted and the GLS regression model without correlation parameters was performed (coefficients in Table 4). This revealed an intercept of 47.702, the pre-slope value as -0.315 , a significant immediate change in level in July 2013 as 
$11.532(p=0.004)$, and a non-significant change between pre $(-0.315)$ and post $(-0.106)$ slopes of $0.208(p=0.258)$. Before the passage of justice reinvestment in Oregon, the number of individuals being admitted to probation each month in the medium usage county appears to have been in a state of moderate decline. However, after the bill was enacted, there is a significant increase in the use of prison admissions and the slope changed to decay less rapidly, supporting $\mathrm{H}_{2}$. This shows that the absolute change between the counterfactual and the fitted values in July 2016 is an increase of $19(78.2 \%)$ people being admitted to probation. Contrary to the the high usage county results, the medium usage proxy estimated total change is an increase of 531 individuals admitted to probation after the passage of HB 3194 .

There were sections of the data missing in the probation admissions data sets for the low usage county likely due to the sparse offending population in this rural county. As with the prison admissions data set, no autocorrelation was found when individual ACF and PACF plots were made. All ARIMA $(0,0,0)$ model residuals were no different than white noise (see Table 2). The regression model (without ARMA) of probation admissions predicted that the intercept is 7.981 , the pre slope is -0.057 , a non-significant change of level of $2.115(p=0.182)$ in July 2013, and a non-significant change in slope from pre ($0.057)$ to post $(0.019)$ of $0.037(p=0.607)$. As neither the slope or level changes were significant, it is concluded that probation admissions exhibited no changes after the passage of justice reinvestment. However, the estimated total change of the post justice reinvestment period was an increase of 97 individuals admitted to probation despite nonsignificant changes in the data level or trends. 
Table 5. Hypothesis 3, Regression Coefficients and Absolute \& Relative Change

\begin{tabular}{|c|c|c|c|c|c|c|c|}
\hline & & \multicolumn{3}{|c|}{$\begin{array}{c}\text { Dependent Time Series (ARMA Model) } \\
\text { State of Oregon } \\
\text { Community Supervision }(2,3)\end{array}$} & \multicolumn{3}{|c|}{$\begin{array}{c}\text { Dependent Time Series (ARMA Model) } \\
\text { High Usage County } \\
\text { Community Supervision }(0,0)\end{array}$} \\
\hline & & Value & (Std. Error) & t-value & Value & (Std. Error) & $\mathrm{t}$-value \\
\hline \multirow{4}{*}{$\begin{array}{l}\text { Regression } \\
\text { Coefficients }\end{array}$} & Intercept & 1173.46 & (25.16) & $46.63 * * *$ & 242.95 & (7.53) & $32.22 * * *$ \\
\hline & Time & -0.26 & (1.15) & -0.22 & -0.35 & $(0.35)$ & -1.01 \\
\hline & Ilevel & 41.41 & (32.15) & 1.29 & 21.84 & (10.91) & $2.00 *$ \\
\hline & Trend & -0.95 & (2.01) & -0.47 & -1.44 & $(0.58)$ & $-2.45 * *$ \\
\hline \multirow{5}{*}{ Change } & Absolute & 13.9 & & & \multicolumn{3}{|l|}{-19.9} \\
\hline & Relative & $1.21 \%$ & & & \multicolumn{3}{|l|}{$-9.1 \%$} \\
\hline & Total & -774 & & & \multicolumn{3}{|l|}{-14} \\
\hline & & \multicolumn{3}{|c|}{$\begin{array}{c}\text { Medium Usage County } \\
\text { Community Supervision }(1,1)\end{array}$} & \multicolumn{3}{|c|}{$\begin{array}{c}\text { Low Usage County } \\
\text { Community Supervision }(0,0)\end{array}$} \\
\hline & & Value & (Std. Error) & $\mathrm{t}$-value & Value & (Std. Error) & $\mathrm{t}$-value \\
\hline \multirow{4}{*}{$\begin{array}{l}\text { Regression } \\
\text { Coefficients }\end{array}$} & Intercept & 51.48 & $(3.51)$ & $14.65 * * *$ & 7.41 & $(0.92)$ & $8.07 * * *$ \\
\hline & Time & -0.29 & $(0.16)$ & -1.81 & -0.03 & $(0.04)$ & -0.73 \\
\hline & Ilevel & 10.40 & (4.88) & $2.12 *$ & 2.01 & (1.33) & 1.50 \\
\hline & Trend & 0.19 & $(0.27)$ & 0.71 & -0.004 & $(0.07)$ & -0.06 \\
\hline \multirow{3}{*}{ Change } & Absolute & 16.1 & & & 1.88 & & \\
\hline & Relative & $50.5 \%$ & & & $35.3 \%$ & & \\
\hline & Total & 370 & & & 54 & & \\
\hline
\end{tabular}

H3 - After the introduction of HB 3194, the community supervision populations will increase.

The State's community supervision populations were best modeled by an ARIMA $(2,0,3)$ after ACF and PACF plots were made, showing both autoregressive and moving average errors. The model is concluded as the best fit as all parameters were significant as show in Table 2, and the model residuals were white noise $\left(\mathrm{X}^{2}=35.861, \mathrm{df}=25, p=\right.$ 0.073). The GLS - ML regression with ARMA (2,3) coefficients (Table 5), produced an intercept of 1173.458 , a pre-intervention slope of -0.258 which is near flat, a nonsignificant intervention level change of $41.415(p=0.202)$, and a non-significant change in slope of $-0.9473(p=0.639)$. The post slope was calculated to be -1.21 . The count of individuals on probation or parole by month shows similar trends as probation admissions. 


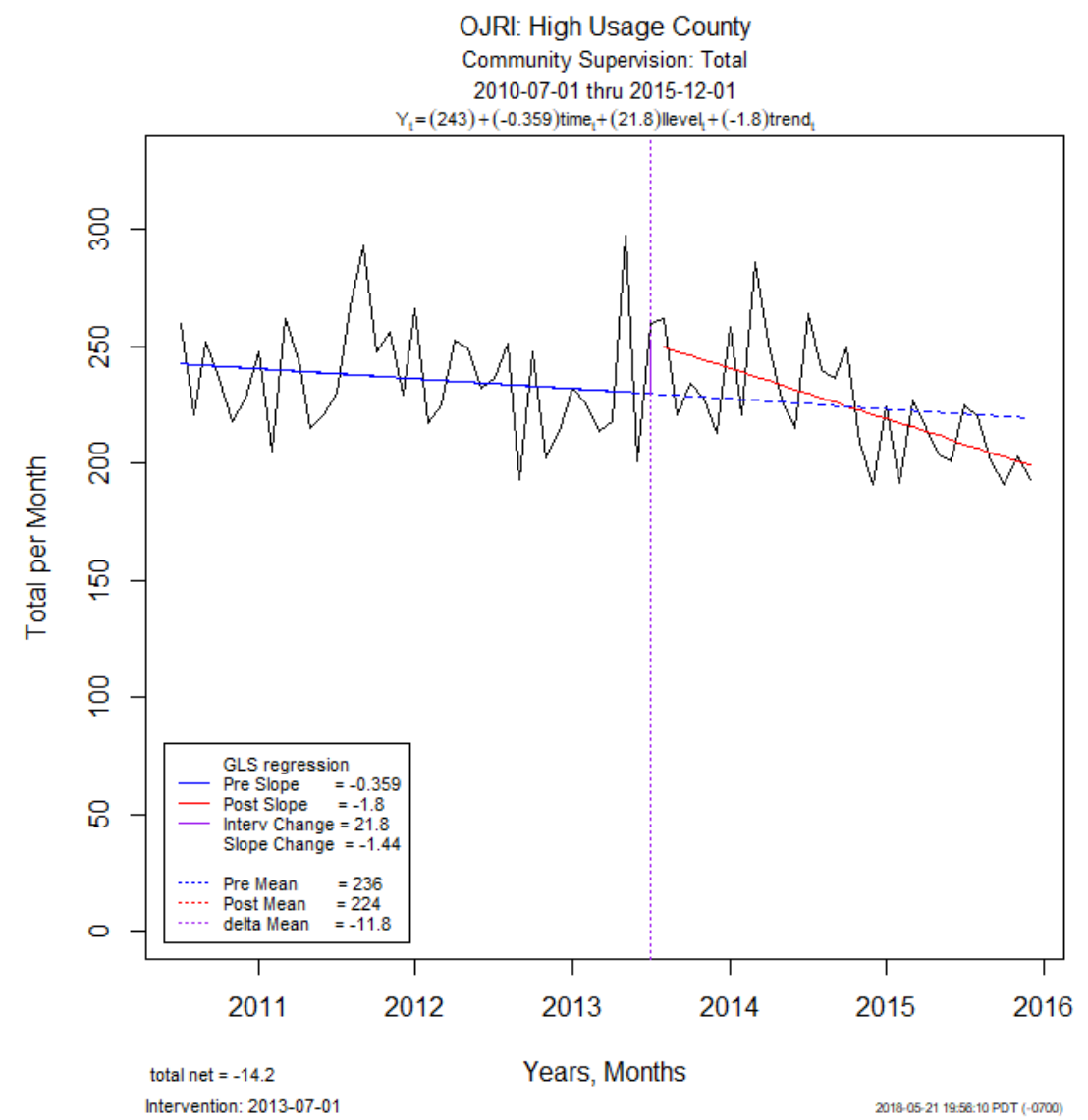

Figure 6. The High Usage County Community Supervision Population, July 2010 to December 2015

Both the pre-slope and the post-slope are in decline, with a small increase after the enrollment of the law. However, neither of these changes in the post-intervention period are significant. The calculation of the estimated total change after the enrollment of HB 3194 is an increase of 774 people under community supervision in Oregon. The absolute change as of December 2015 was 13.9 (1.21\%) from the end of the counterfactual projection of the pre time series.

Individuals admitted to community supervision in the high usage county (Figure 6) did not show serial autocorrelation when the ACF and PACF plots were made. The BoxLjung test on the ARIMA $(0,0,0)$ model reveals that the residuals were not different from white noise $\left(X^{2}=20.495, \mathrm{df}=25, p=0.720\right)$. Therefore, the model is accepted as a good 
fit. The GLS regression without ARMA parameters (coefficients shown in Table 5) modeled the intercept to be 242.950 , the pre-intervention slope as -0.359 , a significant immediate change of level at the time of the intervention of $21.842(p=0.049)$, and a significant change between the pre-intervention slope (-0.359) and the post-intervention slope $(-1.800)$ of $-1.441(p=0.016)$. In figure 5 , the analysis reveals a slight decline in the combined probation and parole population with a significant level change at the time of the intervention. The post slope rapidly decreases, where the absolute change between the actual and counterfactual values in December 2016 was a decrease of $19.9(-9.1 \%)$ individuals on community supervision. Overall, the estimated total change during the postintervention period is a decrease of 14 individuals on community supervision. This provides mixed support for $\mathrm{H}_{3}$ because the initial increase is significant but the population trend moves in the opposite direction at a greater rate.

The total number of individuals on community supervision in the medium usage county (figure 7) contained autocorrelation in the ACF and PACF plots. An ARIMA $(1,0,1)$ model best filters the data: the AR and MA parameters were both significant (Table 2) and the residuals were no different than white noise $\left(\mathrm{X}^{2}=25.732 \mathrm{df}=25, p=0.422\right)$ and the model is accepted. As shown in Table 5, the GLS regression with ARMA $(1,1)$ predicted the intercept at 51.488, a pre-intervention slope of -0.297 , a significant immediate change at the intervention of $10.406(p=0.037)$, and a non- significant change between the preslope $(-0.297)$ and post-slope $(-0.102)$ of $0.195(p=0.479)$. The community supervision population (probation and parole) exhibit similar trends and trend changes as the probation admission data. There is an increase of individuals on community supervision directly after the intervention, and the rate of decay decreases, suggesting that more individuals are under 
community supervision $\left(\mathrm{H}_{3}\right)$. The absolute change in December 2015 between the counterfactual and fitted values was an increase of $16.1(50.5 \%)$ people on community supervision. Furthermore, the estimated total change is an increase of 370 individuals on community supervision in the medium usage county.

Finally, no data were missing from the community supervision data set. ACF and PACF plots showed no autocorrelation in the data, and the residuals were no different than white noise (Table 2). The low usage county's correctional supervision GLS regression (without ARMA) revealed an intercept of 7.419, a pre slope of -0.031 , a non-significant change in level of $2.008(p=0.136)$, and a non-significant change in slope from pre and post $(-0.035)$ values of $-0.004(p=0.951)$. In conjunction with the other two low usage county analyses, there appears to be no change on community supervision populations after HB 3194 (2013) was passed. Overall, I estimate the total change between July 2013 and December 2015 to be an increase of 54 people on community supervision in the low usage county. 


\section{Discussion}

Oregon's House Bill 3194 (2013) restructured parts of the criminal code: reclassifying driving infractions, reducing the penalty for marijuana offenses and increased transitional leave. The goal of this was to reduce the number of people the state was sending to prison by increasing the use of evidence-based programs on both the front-end and the back-end of the court system. Increases in probation or parole would suggest the success of implementation and county buy-in. The law also includes the Oregon Justice Reinvestment grant to aid counties in funding programs that are outside their budget. Decreases in custody populations (prison or jail) would also suggest the success of the bill: if rehabilitation and diverted sentence programs are effective, less individuals will be sentenced to serve time. Less individuals serving time translates to dollars saved, and more funding for OJRG programs. In this study, levels of implementation have been assigned by the number of OJRG programs in use with a high of 12 in the high usage county, a medium of 7 in the medium usage proxy, and a low of 2 in the low usage county.

The Bureau of Justice Assistance assessed eight states where the Justice Reinvestment Initiative had assisted in the construction of justice reinvestment law which had been in effect for one year. The report revealed that all of the states: Arkansas, Hawaii, Louisiana, Kentucky, New Hampshire, North Carolina, Ohio, and South Carolina, had seen substantial decreases in their prison populations. The fiscal impact report from the legislative fiscal office (2013) called for a reduction in prison bed counts by 700 over the first biennium and by another 800 beds in the second biennium after the law's passage (LFO, 2013). The state of Oregon has achieved an estimated decrease of 670 offenders being admitted to prison after the introduction of justice reinvestment. The goals of the first 
biennium have very nearly been met, yet it has taken an additional year to achieve. The high usage proxy achieved the only significant change between the pre and post periods, with a substantial drop immediately and a decreased trend of prison admissions. I estimate that this resulted in the high usage county decreasing their prison admissions by 778 individuals. The two other county proxies, the medium usage and the low usage, did not achieve substantial changes and had slight increases in prison admissions.

Therefore, I find support on the state level and conditional support by counties for hypothesis 1 . The county results make direct assertions more complicated. The high usage proxy county has significant trend changes between the pre and post intervention periods in the expected direction. The medium usage county prison admissions do not show any significant changes in either immediate impact on the data at the date of the intervention or trend change between the pre- and post-intervention periods. This could be the result of the medium usage county focusing on back-end (parole) programing after HB 3194 was enrolled. Furthermore, the low usage county prison admissions show no change in either immediate impact or pre-post trend change. This failure to reach statistical significance could reflect statistical power and low base rate limitations, but also could reflect that given that each county has similar rates across all measures a critical threshold of programming may be required to be met before justice reinvestment can be effective. It appears that the high usage county, which has one of the highest populations in Oregon and implementation level, affects state trends for prison admissions but may be dampened by lower usage counties. Ultimately, prison admissions have declined in Oregon after the passage of HB 3194. 
I also analyze probation admissions in the evaluation of other measures that could provide evidence for the effectiveness of justice reinvestment. Previous research has not examined probation admissions directly or the number of individuals on community supervision. Hypothesis 2, that probation admissions will increase, is supported by the overall state increase in probation admissions. Probation admissions at the state level show that there was an immediate impact from the passage of justice reinvestment which resulted in the estimated total increase of 1,300 individuals admitted to probation in the State. The results provide evidence that justice reinvestment is deferring more individuals to probation across the state.

Again, analyses of county probations provide conflicting results. The high usage county data reflect opposite results to the State. The data reveal no significant immediate impact however the change of slope between pre and post is significant. This amounts to an estimated 900 person decrease by the end of the study period. The high usage proxy appears to be admitting less people to probation which does not support hypothesis 2 . This could be due to a focus by the high usage county justice reinvestment programming on intensive supervision or a higher frequency of jail usage. Further study is required to understand the decreases in the high usage county's probation admissions. Additionally, the decline in probation admissions could be affecting the overall community supervision population: if parole admissions increased after justice reinvestment but then remained stable (only level change), these effects might be washed out by stark declines in probation admissions. As the community supervision measure is a combination of probation and parole, future research should investigate parole admissions as well. 
The medium usage county's probation admission data indicate a significant immediate impact with an increase of about 12 people without a significant trend change between the pre- and post-periods. However, before the passage of justice reinvestment probation admissions were in decline. After the passage of HB 3194 (2013), the rate of decline slowed. By the end of the study period, this amounts to an estimated 531 person increase of admissions to probation than if justice reinvestment had not been enrolled. The low usage county's probation admission data did not show any significant change in immediate level or trend, but does mirror the State and the medium usage results. The medium usage and similar counties appear to have a sustained increase in level of probation admissions and an increase in the rate of use of probation. These increases appear to be influencing the state data. Additionally, because the high usage county has about $20 \%$ of the state population and about $20 \%$ of the admissions, the high usage's substantial drop in trend could be moderating the state trend.

I also find moderate support for hypothesis 3. The total number of individuals on community supervision at the state level has no significant change in either immediate impact or trend change. Despite not achieving significance, I estimate there has about 770 more individuals under probation and parole across the State. However, there is significant immediate impact and trend change for the high usage county which results in an estimated total decrease of 14 people under community supervision. Alternatively, the medium usage data show that before the passage of justice reinvestment community supervision was declining and increased dramatically after the bill was passed. The low usage county community corrections data does not reveal any significant changes in impact or trend. 
There is a minor increase in level at the intervention date but the trend of the post period is nearly identical that of the pre-period. 


\section{Limitations}

As with all research, this study's findings should not be taken without considering the context of its limitations. The first limitation is the assumption that was made to maintain the integrity of the time series frequency for the low usage county's prison admissions data in allocating zeros to all missing data. In addition, the low base rate and statistical power of the low usage county make the interpretation of these results nearly difficult. If this assumption is incorrect, and there was simply no reporting for the missing months, then this could be masking real values greater than zero. Furthermore, as Hinkle and colleagues describe, with small counties (or political units), measurement is difficult because of low base rates and low statistical power (Hinkle et. al, 2013). Second, collecting community supervision and recidivism data beginning at the 2010 cohort meant that there was a major phase-in period of a year and a half before the data stabilized. When the number of recidivists per month was calculated it revealed a curvilinear increase from July 2010 to June of 2012 beginning at zero. Any regression performed on this data would be significantly influenced by this phase-in period; it would produce erroneous calculations of the pre time series intercept and slope (example included in Appendix E). This masks any real trend the pre-intervention period might have had and therefore makes analysis impossible.

Not having access to financial data for the counties' expenditures on evidencebased programing prevents this from being an encompassing evaluation of the outcome measures of HB 3194 (2013). The law calls for $75 \%$ of all county expenditure on programming to be evidence-based. The requirement for empirical support of any particular treatment or program mandates that each county will implement what works. 
The OJR grant can be a majority of that percentage, or it could be far less. An example of this would be if a county has already been funding programs that are evidence-based and uses the OJR grant for two new programs in the 2015-2017 biennium. Access to financial data would be a more accurate way to measure implementation of justice reinvestment.

There also exists a "black-box" problem with this study of justice reinvestment. Without process/ implementation evaluations, this study cannot say how or why HB 3194 is producing the effects that are seen in the analyses. For example, Multnomah County receives $\$ 1.3$ million from the OJR grant for each of three subtypes: alcohol and drug treatment, supervision, and mentoring services. For the $\$ 1.3$ million received for alcohol and drug treatment, this could fund one large program or several smaller programs. The variance in programming could produce inconsistent effects on the measured populations. Furthermore, programs that are being implemented may not be following fidelity to the theory of the program. The results of justice reinvestment depend heavily on program fidelity across the state and counties. If a county uses an evidence-based model for a new program but does not implement correctly, the program may produce iatrogenic effects. Additionally, not all counties roll out their programs at the same time. This could result in a lag in the implementation of justice reinvestment, which would be interpreted as findings of no significance effect within the study period.

Finally, confining the dependent variables to those explicitly stated in HB 3194 (2013) may limit further understanding of the impact of the intervention. System thermodynamics (Wright and Rosky, 2011) suggests that added pressure of change in the system will affect other microsystems not directly targeted or addressed by the bill. For example, if more individuals are being released into community corrections and 
rehabilitation programs on parole or probation, logically it would follow that case numbers would rise for probation or parole officers (unless more officers are hired), adding additional stresses which could translate to increased violations in order to decrease case numbers. Population changes could also affect the measures by increasing the number of individuals convicted of crime. This could result in higher numbers of individuals being admitted to prison or probation and increase community supervision populations. 
Conclusion

Sentencing law changes beginning in the 1970s have compounded to produce an approximately $350 \%$ increase in the incarcerated populations of the United States. Total expenditures for federal corrections had risen above $\$ 51$ billion by 2012 (BJA, 2014). In Oregon, the incarcerated population has increased nearly 50\%, from 9,491 in 2000 to 13,784 in 2010 (BJA, 2014). The revolving door effect will continue to create an issue for federal, state, and local justice systems. This study suggests that justice reinvestment in Oregon appears to achieve its goals in many ways, but the magnitude depends on each county and its implementation adherence. Overall, prison admissions and probation admissions in the state have changed in the intended direction of justice reinvestment. However, the county level results show a variety of outcomes that are produced by the law. The policy implications of this are that justice reinvestment is producing reductions in admissions, but continuance of this depends on the level of utilization of the grant. For each county, policies should be adopted that focus on their individual needs that reinforce and support evidence-based programing. Furthermore, while initial success of HB 3194 has been seen in the three years after its enrollment, counties are still implementing justice reinvestment. An example of this is the low usage proxy, which increased the number of programs for which it received funding for by 5 in two years. Further implementation throughout Oregon will most likely continue to produce an actualization of the goals set out by the legislature. This will have increased returns for the state aggregate totals, and therefore continue to produce savings throughout Department of Corrections budgets. However, according to Tucker and Cadora (2013), rehabilitation and reentry services are only one of four areas of reinvestment that can reduce recidivism. While the returns on this 
investment might be lagged by many years, further funding should be considered for allocation to mental health, education, and community development as crime prevention tactics.

By providing assistance to counties to utilize evidence-based practices for the program subtypes I argue that these findings have a combined effect on the total supervision population of Oregon. Justice reinvestment has reduced incarcerated populations and increased incarceration deferment and community supervision populations. The direction of future research should focus on determining the effect of justice reinvestment on the total number of individuals under state supervision, if there is an effect on public safety, measuring the effect of the bill on recidivism, and determining the change of county expenditures on evidence-based programming. 
References

Achim Zeileis, Torsten Hothorn (2002). Diagnostic Checking in Regression Relationships. R News 2(3), 7-10. URL https://CRAN.R-project.org/doc/Rnews/

Alexander, M. (2012). The new Jim Crow: Mass incarceration in the age of colorblindness. The New Press.

Austin, J., Clark, J., Hardyman, P., \& Henry, D. A. (1998). Three strikes and you're out: The implementation and impact of strike laws. Washington DC: National Institute of Justice- $\quad$ US Department of Justice.

Austin, J., Cadora, E., Clear, T. R., Dansky, K., Greene, J., Gupta, V., ... \& Young, M. C. (2013). Ending mass incarceration: Charting a new justice reinvestment. Washington, DC: The Sentencing Project.

Berry, W. D., \& Lewis-Beck, M. S. (1986). New tools for social scientists: Advances and applications in research methods. Sage Publications, Inc.

Bird, M., \& Grattet, R. (2015). Policy change and recidivism: The effects of California's realignment and local implementation strategies on rearrest and reconviction. Criminal Justice Policy Review, 0887403415604900.

Briggs, C. S., Sundt, J. L., \& Castellano, T. C. (2003). The effect of supermaximum security prisons on aggregate levels of institutional violence. Criminology, 41(4), 1341-1376.

Britt, C. L., Kleck, G., \& Bordua, D. J. (1996). A reassessment of the DC gun law: Some cautionary notes on the use of interrupted time series designs for policy impact assessment. Law and Society Review, 361-380.

Bureau of Justice Assistance. (2014). Justice Reinvestment Initiative State Assessment Report. Bureau of Justice Assistance.

Cahalan, M. (1986). Historical Corrections Statistics in the United States, 1850- 1984 (NCJ - 102529, December 1986) (U.S. Department of Justice, Bureau of Justice Statistics).

Campbell, C. M. (2015). Popular punitivism: Finding a balance between the politics, presentation, and fear of crime. Sociology Compass, 9(3), 180-195.

Campbell, D. T., \& Stanley, J. C. (1963). Experimental designs for research on teaching. Handbook of research on teaching, 171-246.

Carson, A., \& Anderson, E. (2016). Prisoners in 2015 (NCJ - 250229., Prisoner Series) (U.S. Department of Justice, Bureau of Justice Statistics). Prisoner Series: http://www.bjs.gov/index.cfm?ty=pbdetail\&iid=5869

Chamberlain, A. W., \& Wallace, D. (2016). Mass reentry, neighborhood context and recidivism: Examining how the distribution of parolees within and across neighborhoods impacts recidivism. Justice Quarterly, 33(5), 912-941. 
Clear, T. R. (2009). Imprisoning communities: How mass incarceration makes disadvantaged neighborhoods worse. Oxford University Press.

Clinton, William J. (1994) "Address Before a Joint Session of the Congress on the State of the Union," January 25, 1994. Online by Gerhard Peters and John T. Woolley, the American Presidency Project. http://www.presidency.ucsb.edu/ws/?pid=50409.

Cohen, R. L. (1991). Prisoners in 1990 (NIJ - 129198, Prisoner Series) (U.S. Department of Justice, Bureau of Justice Statistics). Prisoner Series: http://www.bjs.gov/index.cfm?ty=pbdetail\&iid=4042

Counsel of State Governments, Justice Center. (2017). Justice Reinvestment: Connecticut Overview. https://csgjusticecenter.org/jr/ct/

Cullen, F. T., \& Gendreau, P. (2001). From nothing works to what works: Changing professional ideology in the 21st century. The Prison Journal, 81(3), 313-338.

Fabelo, T., Osher, F. C., \& Thompson, M. (2007). Justice reinvestment: Increasing public safety and managing the growth of Pennsylvania prison population.

Field, A. (2013). Discovering statistics using IBM SPSS statistics. sage.

Fox, J., \& Weisberg, S. (2011). An R companion to applied regression: Time-Series Regression and Generalized Least Squares in R: An Appendix to An R Companion to Applied Regression, Second Edition. Sage Publications.

Gainey, R. R., Payne, B. K., \& O'Toole, M. (2000). The relationships between time in jail, time on electronic monitoring, and recidivism: An event history analysis of a jail-based program. Justice Quarterly, 17(4), 733-752.

Hagan, J., \& Coleman, J. P. (2001). Returning captives of the American war on drugs: Issues of community and family reentry. NCCD news, 47(3), 352-367.

Hepburn, J. R., \& Albonetti, C. A. (1994). Recidivism among drug offenders: A survival analysis of the effects of offender characteristics, type of offense, and two types of intervention. Journal of Quantitative Criminology, 10(2), 159-179.

Hinkle, J. C., Weisburd, D., Famega, C., \& Ready, J. (2013). The problem is not just sample size: the consequences of low base rates in policing experiments in smaller cities. Evaluation review, 37(3-4), 213-238.

Hyndman RJ (2017). _forecast: Forecasting functions for time series and linear models_. R package version 8.2, <URL: http://pkg.robjhyndman.com/forecast>.

Hyndman RJ and Khandakar Y (2008). "Automatic time series forecasting: the forecast package for R.”_Journal of Statistical Software_, *26*(3), pp. 1-22. <URL: http://www.jstatsoft.org/article/view/v027i03>.

Jensen, E. L., \& Metsger, L. K. (1994). A test of the deterrent effect of legislative waiver on violent juvenile crime. NCCD news, 40(1), 96-104.

Jensen, E. L., Gerber, J., \& Mosher, C. (2004). Social consequences of the war on drugs: the legacy of failed policy. Criminal Justice Policy Review, 15(1), 100-121. 
John Fox and Sanford Weisberg (2011). An $\{\mathrm{R}\}$ Companion to Applied Regression, Second Edition. Thousand Oaks CA: Sage. URL:

http://socserv.socsci.mcmaster.ca/jfox/Books/Companion

Kubrin, C. E., Squires, G., \& Stewart, E. (2007). Neighborhoods, race, and recidivism: The Community reoffending nexus and its implications for African Americans.

Latessa, E. J., \& Lovins, B. (2010). The role of offender risk assessment: A policy maker guide. Victims and Offenders, 5(3), 203-219.

Legislative Fiscal Office. (2013). Seventy-Seventh Oregon Legislative Assembly - 2013 Regular Session, FISCAL IMPACT OF PROPOSED LEGISLATION Measure: HB 3194 - A. Salem, OR: Oregon State Legislature.

Listwan, S. J., Jonson, C. L., Cullen, F. T., \& Latessa, E. J. (2008). Cracks in the penal harm movement: Evidence from the field. Criminology \& Public Policy, 7(3), 423-465.

Lynch, J. P., \& Sabol, W. J. (2004). Assessing the effects of mass incarceration on informal social control in communities. Criminology \& Public Policy, 3(2), $267-$ 294.

Martinson, R. (1974). What works? -Questions and answers about prison reform. The public interest, (35), 22.

Marvell, T. B., \& Moody, C. E. (1996). Determinate sentencing and abolishing parole: The long-term impacts on prisons and crime. Criminology, 34(1), 107-128.

McDowall, D. (1980). Interrupted time series analysis (Vol. 21). Sage.

Multnomah County Justice Reinvestment Program. (2015). Quarterly report: Fy 15 q 4. Portland, OR: MCJRP from: https://multco.us/file/46312/download

Multnomah County Justice Reinvestment Program. (2017). Examining the Implementation of Justice Reinvestment in Multnomah County: Measurement, Preliminary Analysis, and Future Evaluations. Portland, OR: MCJRP from: https://multco.us/file/57700/download

Nixon, R. (1971). Remarks About an Intensified Program for Drug Abuse Prevention and Control. June 17, 1971. Online by Gerhard Peters and John T. Woolley, The American Presidency Project. http://www.presidency.ucsb.edu/ws/?pid=3047.

Oregon Criminal Justice Commission. (2017). 2015-2017 Justice Reinvestment Grant Funding by County. Retrieved from http://www.oregon.gov/cjc/data/Pages/jri.aspx

Oregon Criminal Justice Commission. (2018). Interactive Data: Oregon Uniform Crime Report. Retrieved from http://www.oregon.gov/cjc/data/Pages/OUCR.aspx

Orrick, E. A., \& Vieraitis, L. M. (2015). The Cost of Incarceration in Texas: Estimating the Benefits of Reducing the Prison Population. American Journal of Criminal Justice, 40(2), 399-415.

Pettit, B., \& Western, B. (2004). Mass imprisonment and the life course: Race and class inequality in US incarceration. American sociological review, 69(2), 151-169. 
Pinheiro J, Bates D, DebRoy S, Sarkar D and R Core Team (2018)._nlme: Linear and Nonlinear Mixed Effects Models_. R package version 3.1-131.1, <URL: https://CRAN.R-project.org/package=nlme>.

R Core Team (2017). R: A language and environment for statistical computing. R Foundation for Statistical Computing, Vienna, Austria. URL https://www.Rproject.org/.

Ramirez, J. R., \& Crano, W. D. (2003). Deterrence and Incapacitation: An Interrupted Time-Series Analysis of California's Three-Strikes Law. Journal of Applied Social Psychology, 33(1), 110-144.

Roberts, D. E. (2004). The social and moral cost of mass incarceration in African American communities. Stanford Law Review, 1271-1305.

Sampson, R. J., \& Loeffler, C. (2010). Punishment's place: the local concentration of mass incarceration. Daedalus, 139(3), 20-31.

Schmidt, M. Officer, K. (2015) Justice Reinvestment Implementation in Oregon, August 2013 to April 2015. Oregon Criminal Justice Commission. Retrieved: May $25^{\text {th }}$, 2018, from http://www.oregon.gov/cjc/about/Pages/default.aspx\#publications

Schmidt, M. (2017) Cost Avoidance Report: Per House Bill 3194 (2013). Oregon Criminal Justice Commission. Retrieved: May 25 ${ }^{\text {th }}, 2018$, from http://www.oregon.gov/cjc/about/Pages/default.aspx\#publications

Shepherd, J. M. (2002). Police, prosecutors, criminals, and determinate sentencing: The truth about truth-in-sentencing laws. The Journal of Law and Economics, 45(2), 509-533.

Shumway, R. H., \& Stoffer, D. S. (2011). Time series analysis and its applications. Springer New York.

Simon, J. (2017). Governing through crime. In Law and Poverty (pp. 97-115). Routledge.

Sorensen, J., \& Stemen, D. (2002). The effect of state sentencing policies on incarceration rates. Crime \& Delinquency, 48(3), 456-475.

Spohn, C., \& Holleran, D. (2002). The effect of imprisonment on recidivism rates of felony offenders: A focus on drug offenders. Criminology, 40(2), 329-358.

Sundt, J., Salisbury, E. J., \& Harmon, M. G. (2016). Is downsizing prisons dangerous? Criminology \& Public Policy, 15(2), 315-341.

TFPS. (2016). Task Force on Public Safety. Justice Reinvestment Report to the Legislature, October 2016. Oregon Criminal Justice Commission. Retrieved: May $25^{\text {th }}, 2018$, from http://www.oregon.gov/cjc/about/Pages/default.aspx\#publications

Travis, J., Western, B., \& Redburn, F. S. (2014). The growth of incarceration in the United States: Exploring causes and consequences.

Thompson, H. A. (2010). Why mass incarceration matters: Rethinking crisis, decline, and transformation in postwar American history. The Journal of American History, 97(3), 703-734. 
Tucker, S., \& Cadora, E. (2003). Justice reinvestment: To invest in public safety by reallocating justice dollars to refinance education, housing, healthcare, and jobs [Monograph] Ideas for an Open Society, 3(3).

U.S. Census Bureau. (2018). Annual Estimates of the Resident Population for Counties: April 1, 2010 to July 1, 2016: Oregon. https://www.census.gov/data/tables/2016/demo/popest/counties-total.html

U.S. Department of Housing and Urban Development, Office of Public and Indian Housing. (n.d.). Meeting the Challenge: Public Housing Authorities Respond to the "One Strike You're Out" Initiative. Retrieved September 19, 2017, from https://www.ncjrs.gov/pdffiles1/Photocopy/183952NCJRS.pdf

U.S. Department of Justice. (2017). Prisoners and Prisoner Reentry. Retrieved October 11, 2017, from https://www.justice.gov/archive/fbci/progmenu_reentry.html

Vásquez, B. E., Maddan, S., \& Walker, J. T. (2008). The influence of sex offender registration and notification laws in the United States: A time-series analysis. Crime \& Delinquency, 54(2), 175-192.

Visher, C. A., \& Linster, R. L. (1990). A survival model of pretrial failure. Journal of Quantitative Criminology, 6(2), 153-184.

Visher, C. A., \& Travis, J. (2011). Life on the outside: Returning home after incarceration. The Prison Journal, 91(3_suppl), 102S-119S.

White House, The. (1992) National Drug Control Strategy: A Nation Responds to Drug Use. NCJRS Retrieved April 15, 2018, from https://www.ncjrs.gov/pdffiles1/ondcp/134372.pdf

White House, The. (2016). National Drug Control Budget 2017 highlights. Office of National Drug Control Policy, Retrieved April 15, 2018, from https://obamawhitehouse.archives.gov/ondcp/the-national-drug-control-budget-fy2013-funding-highlights

Wright, K. A., \& Rosky, J. W. (2011). Too early is too soon. Criminology \& Public Policy, 10(4), 881-908.

77th Oregon Legislative Assembly--2013 Regular Session. (2013). House Bill 3194 Enrolled. Salem, OR: Oregon State Legislature. https://olis.leg.state.or.us/liz/2013R1/Downloads/MeasureDocument/HB3194 99th Congress (1986), H.R.5484, 99th Cong., Public Law No: 99-570 H.R.5484 (1986) (enacted). Print. https://www.congress.gov/bill/99th-congress/house-bill/5484 
Appendix A - County Subtype and Populations

Table A1. Oregon Justice Reinvestment Grant Funded Subtypes by County with Population Estimates by County

\begin{tabular}{|c|c|c|}
\hline County & Subtypes & Population Estimate - 2013 \\
\hline Baker County, Oregon & 4 & 16,094 \\
\hline Benton County, Oregon & 5 & 86,066 \\
\hline Clackamas County, Oregon & 8 & 387,995 \\
\hline Clatsop County, Oregon & 8 & 37,093 \\
\hline Columbia County, Oregon & 6 & 49,224 \\
\hline Coos County, Oregon & 6 & 62,563 \\
\hline Crook County, Oregon & 5 & 20,776 \\
\hline Curry County, Oregon & 2 & 22,240 \\
\hline Deschutes County, Oregon & 7 & 165,859 \\
\hline Douglas County, Oregon & 6 & 106,803 \\
\hline Gilliam County, Oregon & 5 & 1,945 \\
\hline Grant County, Oregon & 5 & 7,272 \\
\hline Harney County, Oregon & 3 & 7,175 \\
\hline Hood River County, Oregon & 5 & 22,614 \\
\hline Jackson County, Oregon & 8 & 207,862 \\
\hline Jefferson County, Oregon & 5 & 21,842 \\
\hline Josephine County, Oregon & 5 & 83,268 \\
\hline Klamath County, Oregon & 4 & 65,830 \\
\hline Lake County, Oregon & 4 & 7,806 \\
\hline Lane County, Oregon & 11 & 355,812 \\
\hline Lincoln County, Oregon & 5 & 46,257 \\
\hline Linn County, Oregon & 9 & 118,496 \\
\hline Malheur County, Oregon & 6 & 30,633 \\
\hline Marion County, Oregon & 12 & 321,572 \\
\hline Morrow County, Oregon & 2 & 11,237 \\
\hline Multnomah County, Oregon & 12 & 765,850 \\
\hline Polk County, Oregon & 6 & 76,753 \\
\hline Sherman County, Oregon & 7 & 1,711 \\
\hline Tillamook County, Oregon & 7 & 25,337 \\
\hline Umatilla County, Oregon & 4 & 76,802 \\
\hline Union County, Oregon & 5 & 25,519 \\
\hline Wallowa County, Oregon & 7 & 6,789 \\
\hline Wasco County, Oregon & 4 & 25,458 \\
\hline Washington County, Oregon & 10 & 555,089 \\
\hline Wheeler County, Oregon & 2 & 1,387 \\
\hline Yamhill County, Oregon & 5 & 100,722 \\
\hline
\end{tabular}

Population Source: U.S. Census, 2018

Subtype Source: CJC Interactive Data, 2018 
Appendix B - Normality test

Table B.1 Tests of Normality

\begin{tabular}{lccc|ccc}
\hline & \multicolumn{3}{c}{ Kolmogorov-Smirnova } & \multicolumn{3}{c}{ Shapiro-Wilk } \\
\hline & \multicolumn{1}{c}{ Statistic } & $\frac{\mathrm{df}}{74}$ & $\underline{\text { Sig. }}$ & $\underline{\underline{\text { Statistic }}}$ & $\frac{\mathrm{df}}{74}$ & $\underline{\text { Sig. }}$ \\
State Prison Admissions & 0.069 & 74 & $0.200^{*}$ & 0.985 & 0.529 \\
State Probation Admissions & 0.061 & 74 & $0.200^{*}$ & 0.984 & 74 & 0.473 \\
State Community Supervision & 0.071 & 66 & $0.200^{*}$ & 0.984 & 66 & 0.566 \\
HU Prison Admissions & 0.102 & 74 & 0.056 & 0.966 & 74 & 0.044 \\
HU Probation Admissions & 0.066 & 74 & $0.200^{*}$ & 0.971 & 74 & 0.092 \\
HU Community Supervision & 0.084 & 66 & $0.200^{*}$ & 0.967 & 66 & 0.078 \\
MU Prison Admissions & 0.141 & 74 & 0.001 & 0.973 & 74 & 0.108 \\
MU Probation Admissions & 0.085 & 74 & $0.200^{*}$ & 0.988 & 74 & 0.696 \\
MU Community Supervision & 0.071 & 66 & $0.200^{*}$ & 0.984 & 66 & 0.536 \\
LU Prison Admissions & 0.186 & 74 & 0.000 & 0.884 & 74 & 0.000 \\
LU Probation Admissions & 0.135 & 74 & 0.002 & 0.954 & 74 & 0.009 \\
LU Community Supervision & 0.132 & 66 & 0.006 & 0.956 & 66 & 0.020 \\
\hline
\end{tabular}

$*$ This is a lower bound of the true significance. 


\section{Appendix C - Descriptive Statistics by Year Within Study}

\begin{tabular}{|c|c|c|c|c|c|c|c|}
\hline Prison Admissions & $\mathrm{N}$ & Minimum & Maximum & Std. Deviation & Mean & $\%$ Change & Mean Rate Per 100,000 \\
\hline 2010 & 7 & 65 & 86 & 8.214 & 75.86 & & 10.29 \\
\hline 2011 & 12 & 58 & 98 & 11.733 & 78.25 & $3.15 \%$ & 10.46 \\
\hline 2012 & 12 & 67 & 102 & 9.913 & 84.08 & $7.45 \%$ & 11.08 \\
\hline 2013 & 12 & 68 & 104 & 12.078 & 85.67 & $1.89 \%$ & 11.19 \\
\hline 2014 & 12 & 54 & 104 & 14.841 & 72.42 & $-15.47 \%$ & 9.32 \\
\hline 2015 & 12 & 60 & 85 & 7.012 & 69.58 & $-3.92 \%$ & 8.82 \\
\hline 2016 & 7 & 63 & 80 & 5.598 & 71.00 & $2.04 \%$ & 8.88 \\
\hline Probation Admissions & $\mathrm{N}$ & Minimum & Maximum & Std. Deviation & Mean & $\%$ Change & Mean Rate Per 100,000 \\
\hline 2010 & 7 & 144 & 163 & 7.047 & 153.00 & & 20.75 \\
\hline 2011 & 12 & 129 & 202 & 19.678 & 158.83 & $3.81 \%$ & 21.23 \\
\hline 2012 & 12 & 138 & 185 & 12.803 & 158.50 & $-0.21 \%$ & 20.88 \\
\hline 2013 & 12 & 139 & 213 & 18.466 & 165.42 & $4.37 \%$ & 21.6 \\
\hline 2014 & 12 & 137 & 187 & 16.971 & 164.25 & $-0.71 \%$ & 21.13 \\
\hline 2015 & 12 & 124 & 157 & 10.805 & 138.25 & $-15.83 \%$ & 17.52 \\
\hline 2016 & 7 & 131 & 155 & 7.946 & 141.86 & $2.61 \%$ & 17.74 \\
\hline Community Supervision & $\mathrm{N}$ & Minimum & Maximum & Std. Deviation & Mean & $\%$ Change & Mean Rate Per 100,000 \\
\hline 2010 & 6 & 218 & 260 & 16.999 & 235.83 & & 31.98 \\
\hline 2011 & 12 & 205 & 293 & 24.742 & 243.17 & $3.11 \%$ & 32.5 \\
\hline 2012 & 12 & 193 & 266 & 22.437 & 232.17 & $-4.52 \%$ & 30.59 \\
\hline 2013 & 12 & 201 & 297 & 26.778 & 233.83 & $0.71 \%$ & 30.53 \\
\hline 2014 & 12 & 191 & 286 & 26.431 & 237.33 & $1.50 \%$ & 30.54 \\
\hline 2015 & 12 & 191 & 227 & 13.511 & 208.00 & $-12.36 \%$ & 26.36 \\
\hline
\end{tabular}

Note: Population data was collected from the U.S. Census (U.S. Census, 2018)

Table C2. Medium Usage County Descriptive Statistics by Year

\begin{tabular}{|c|c|c|c|c|c|c|c|}
\hline Prison Admissions & $\mathrm{N}$ & Minimum & Maximum & Std. Deviation & Mean & $\%$ Change & Mean Rate Per 100,000 \\
\hline 2010 & 7 & 7 & 24 & 5.398 & 16.14 & & 10.23 \\
\hline 2011 & 12 & 8 & 20 & 3.988 & 14.08 & $-12.76 \%$ & 8.81 \\
\hline 2012 & 12 & 10 & 21 & 3.175 & 15.42 & $9.52 \%$ & 9.53 \\
\hline 2013 & 12 & 8 & 21 & 4.224 & 14.75 & $-4.35 \%$ & 8.89 \\
\hline 2014 & 12 & 12 & 30 & 4.569 & 17.83 & $20.88 \%$ & 10.48 \\
\hline 2015 & 12 & 10 & 23 & 3.911 & 17.25 & $-3.25 \%$ & 9.86 \\
\hline 2016 & 7 & 14 & 21 & 2.309 & 18.00 & $4.35 \%$ & 9.93 \\
\hline Probation Admissions & $\mathrm{N}$ & Minimum & Maximum & Std. Deviation & Mean & $\%$ Change & Mean Rate Per 100,000 \\
\hline 2010 & 7 & 44 & 58 & 5.21 & 49.86 & & 31.59 \\
\hline 2011 & 12 & 25 & 56 & 9.808 & 40.25 & $-19.27 \%$ & 25.19 \\
\hline 2012 & 12 & 29 & 48 & 5.944 & 39.33 & $-2.29 \%$ & 24.31 \\
\hline 2013 & 12 & 31 & 61 & 7.728 & 41.50 & $5.52 \%$ & 25.02 \\
\hline 2014 & 12 & 33 & 59 & 7.596 & 48.67 & $17.28 \%$ & 28.6 \\
\hline 2015 & 12 & 20 & 57 & 10.633 & 45.17 & $-7.19 \%$ & 25.82 \\
\hline 2016 & 7 & 33 & 58 & 8.58 & 41.57 & $-7.97 \%$ & 22.93 \\
\hline Community Supervision & $\mathrm{N}$ & Minimum & Maximum & Std. Deviation & Mean & $\%$ Change & Mean Rate Per 100,000 \\
\hline 2010 & 6 & 41 & 62 & 7.714 & 53.50 & & 33.9 \\
\hline 2011 & 12 & 35 & 58 & 7.794 & 45.25 & $-15.42 \%$ & 28.32 \\
\hline 2012 & 12 & 28 & 52 & 7.222 & 41.83 & $-7.56 \%$ & 25.86 \\
\hline 2013 & 12 & 35 & 59 & 7.953 & 46.17 & $10.38 \%$ & 27.84 \\
\hline 2014 & 12 & 37 & 72 & 8.569 & 53.83 & $16.59 \%$ & 31.63 \\
\hline 2015 & 12 & 29 & 62 & 9.882 & 47.25 & $-12.22 \%$ & 27.01 \\
\hline
\end{tabular}


Table C3. Low Usage County Descriptive Statistics by Year

\begin{tabular}{|c|c|c|c|c|c|c|c|}
\hline Prison Admissions & $\mathrm{N}$ & Minimum & Maximum & Std. Deviation & Mean & $\%$ Change & Mean Rate Per 100,000 \\
\hline 2010 & 7 & 0 & 8 & 3.094 & 2.29 & & 10.24 \\
\hline 2011 & 12 & 0 & 3 & 0.9 & 0.92 & $-59.83 \%$ & 4.10 \\
\hline 2012 & 12 & 1 & 4 & 0.953 & 2.00 & $117.39 \%$ & 8.99 \\
\hline 2013 & 12 & 0 & 4 & 1.379 & 2.08 & $4.00 \%$ & 9.35 \\
\hline 2014 & 12 & 0 & 3 & 0.985 & 1.67 & $-19.71 \%$ & 7.51 \\
\hline 2015 & 12 & 0 & 4 & 1.165 & 2.42 & $44.91 \%$ & 10.81 \\
\hline 2016 & 7 & 0 & 4 & 1.464 & 2.14 & $-11.57 \%$ & 9.42 \\
\hline Probation Admissions & $\mathrm{N}$ & Minimum & Maximum & Std. Deviation & Mean & $\%$ Change & Mean Rate Per 100,000 \\
\hline 2010 & 7 & 4 & 11 & 2.984 & 7.29 & & 32.59 \\
\hline 2011 & 12 & 1 & 16 & 4.42 & 7.42 & $1.78 \%$ & 33.05 \\
\hline 2012 & 12 & 2 & 13 & 3.055 & 5.67 & $-23.58 \%$ & 25.50 \\
\hline 2013 & 12 & 3 & 13 & 3.157 & 6.83 & $20.46 \%$ & 30.71 \\
\hline 2014 & 12 & 4 & 16 & 3.551 & 9.33 & $36.60 \%$ & 41.96 \\
\hline 2015 & 12 & 2 & 10 & 1.975 & 6.92 & $-25.83 \%$ & 30.90 \\
\hline 2016 & 7 & 3 & 13 & 3.485 & 7.14 & $3.18 \%$ & 31.44 \\
\hline Community Supervision & $\mathrm{N}$ & Minimum & Maximum & Std. Deviation & Mean & $\%$ Change & Mean Rate Per 100,000 \\
\hline 2010 & 6 & 3 & 13 & 3.445 & 7.67 & & 34.29 \\
\hline 2011 & 12 & 3 & 12 & 2.541 & 6.50 & $-15.25 \%$ & 28.95 \\
\hline 2012 & 12 & 3 & 15 & 3.257 & 6.67 & $2.62 \%$ & 30.00 \\
\hline 2013 & 12 & 3 & 11 & 2.575 & 7.42 & $11.24 \%$ & 33.36 \\
\hline 2014 & 12 & 5 & 13 & 2.588 & 8.17 & $10.11 \%$ & 36.74 \\
\hline 2015 & 12 & 3 & 11 & 2.261 & 7.25 & $-11.26 \%$ & 32.37 \\
\hline
\end{tabular}

Note: Population data was collected from the U.S. Census (U.S. Census, 2018) 
Appendix D - Figures of raw data, ACF plots, PACF, and GLS plots

State Figures - Prison Admissions

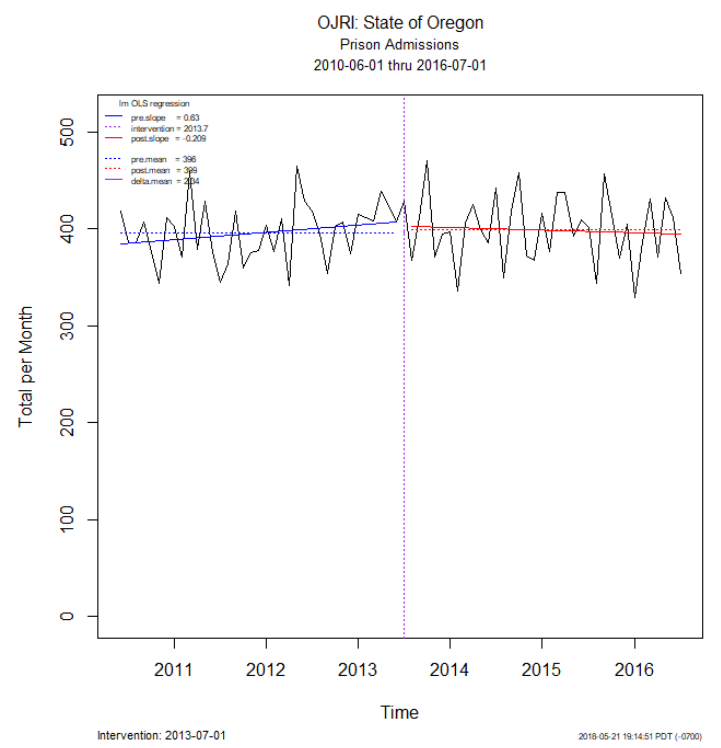

State of Oregon raw data with OLS

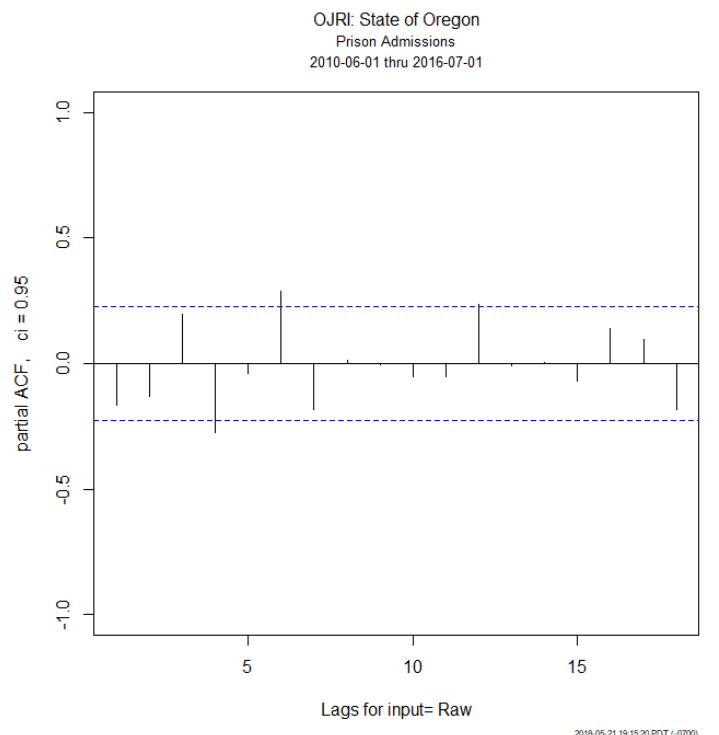

Partial ACF for raw data

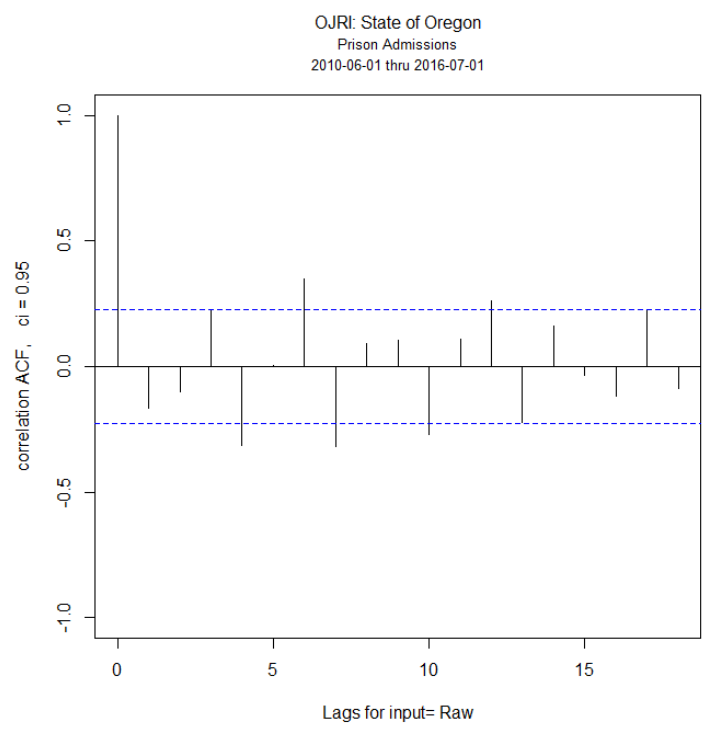

ACF plot for raw data

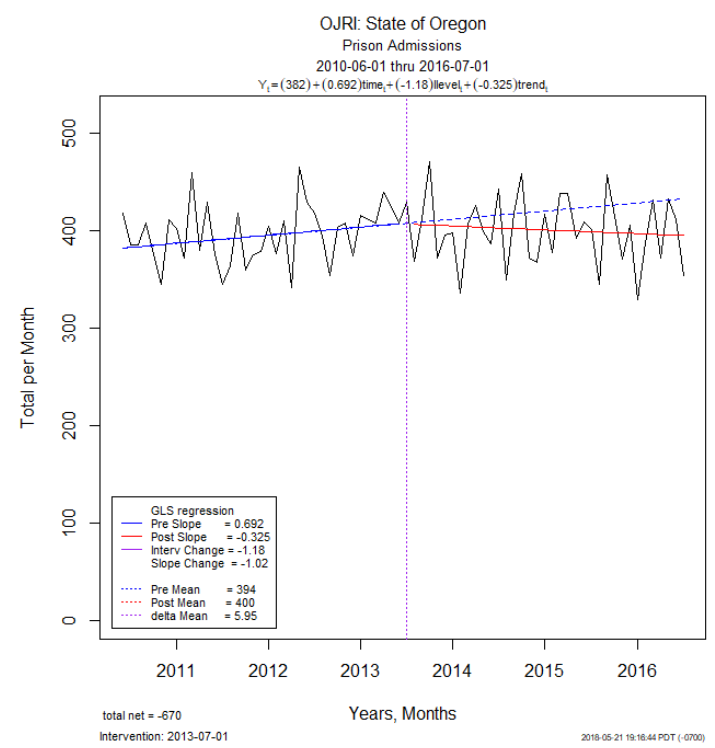

Final model fitted by GLS 
Probation Admissions

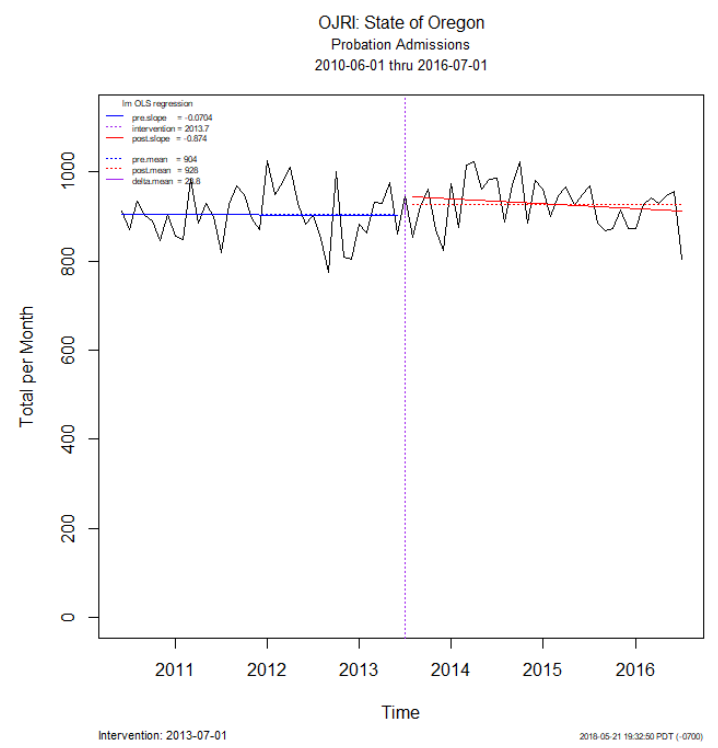

State of Oregon raw data with OLS

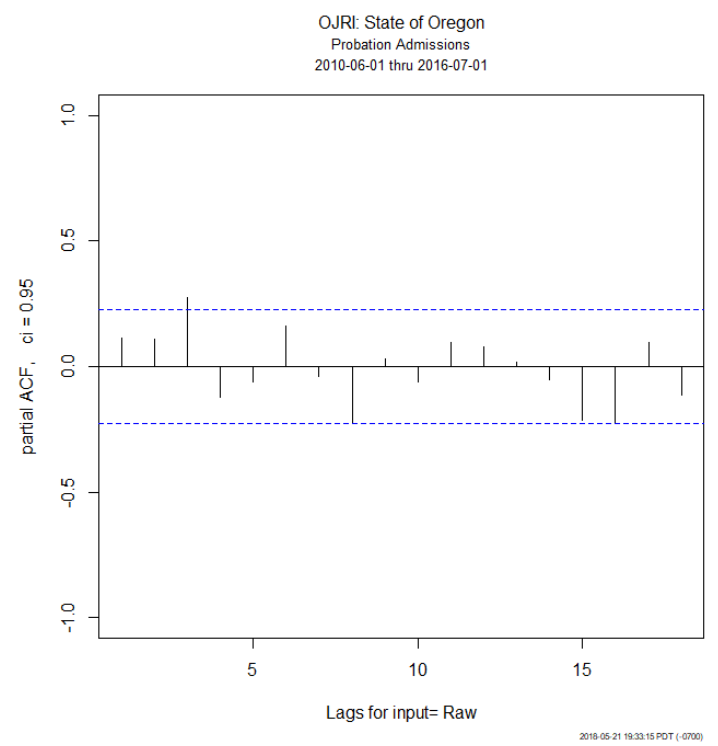

Partial ACF for raw data

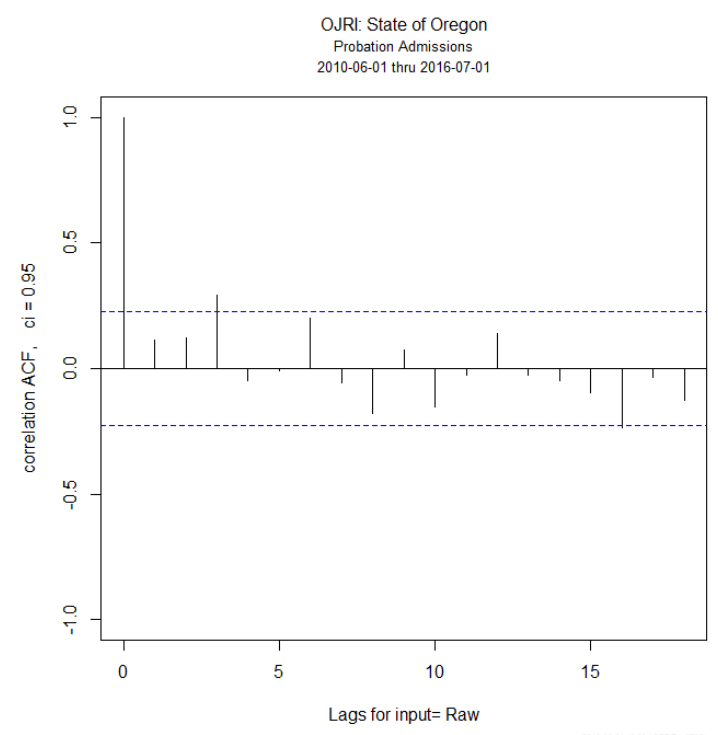

ACF plot for raw data

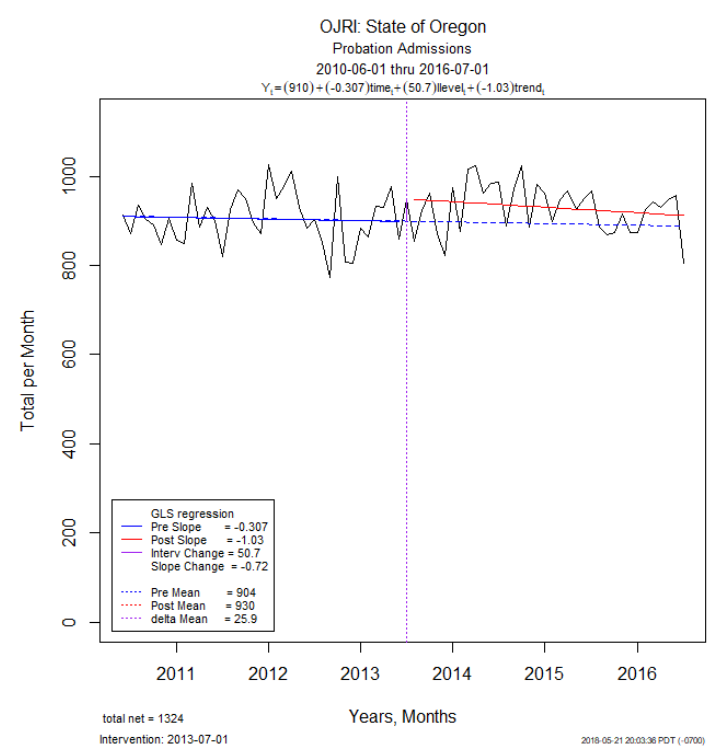

Final model fitted by GLS 
State Community Supervision

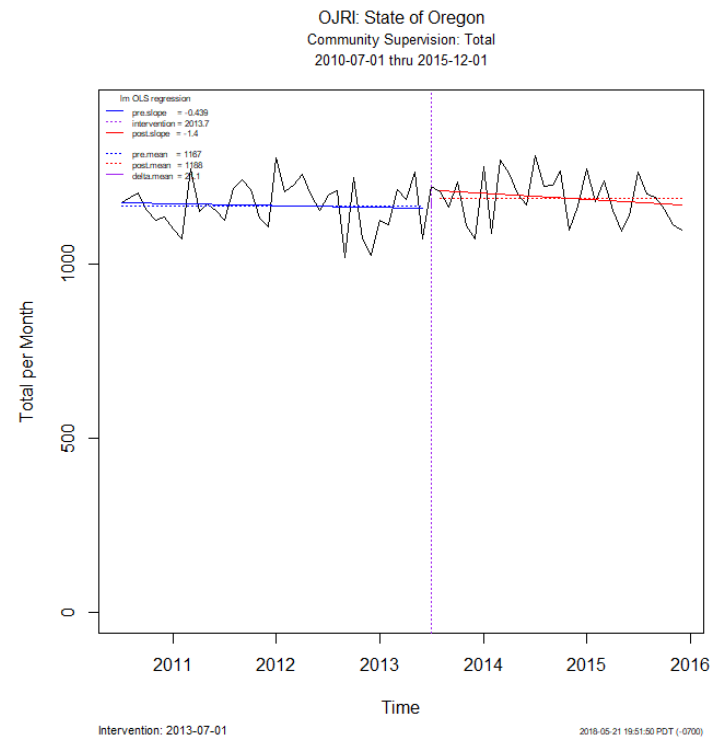

State of Oregon raw data with OLS

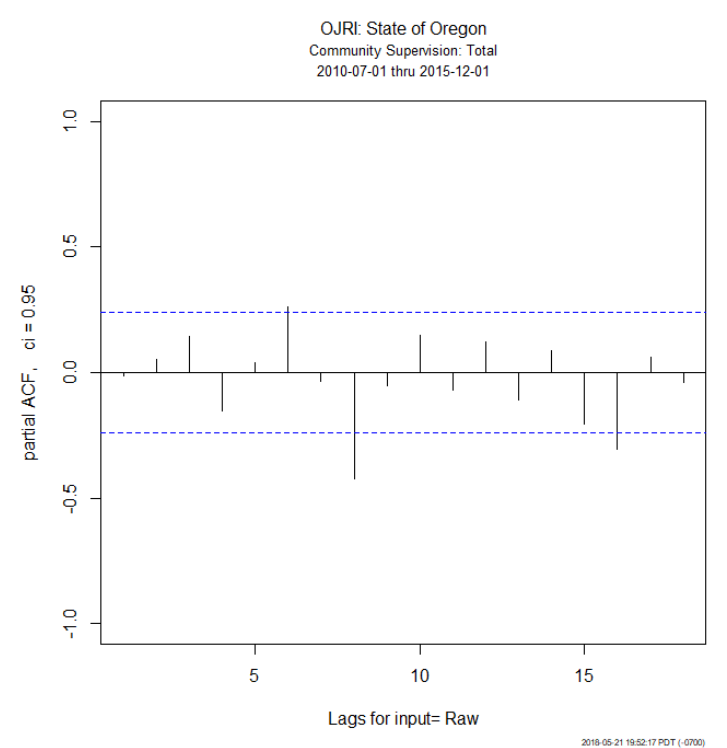

Partial ACF for raw data

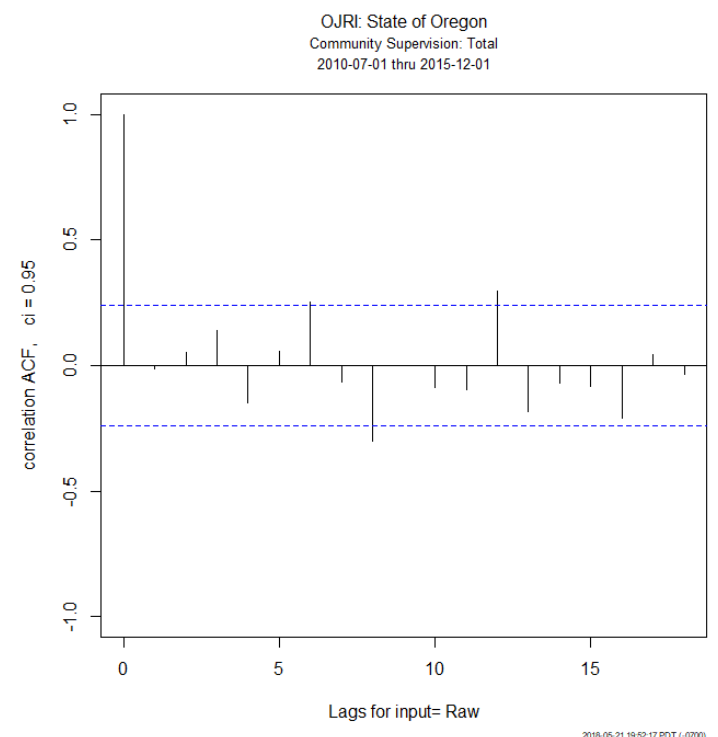

ACF plot for raw data

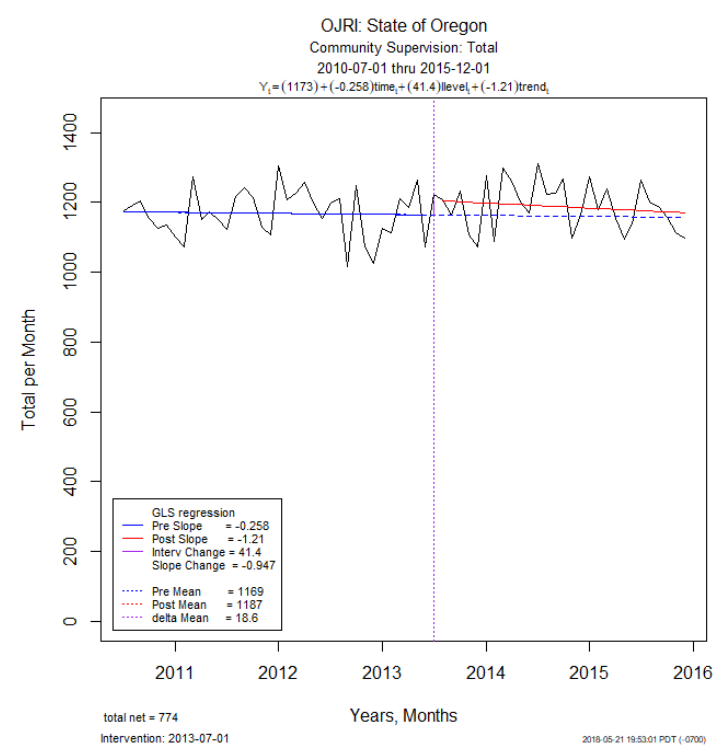

Final model fitted by GLS 


\section{High Usage County}

\section{Prison Admissions}

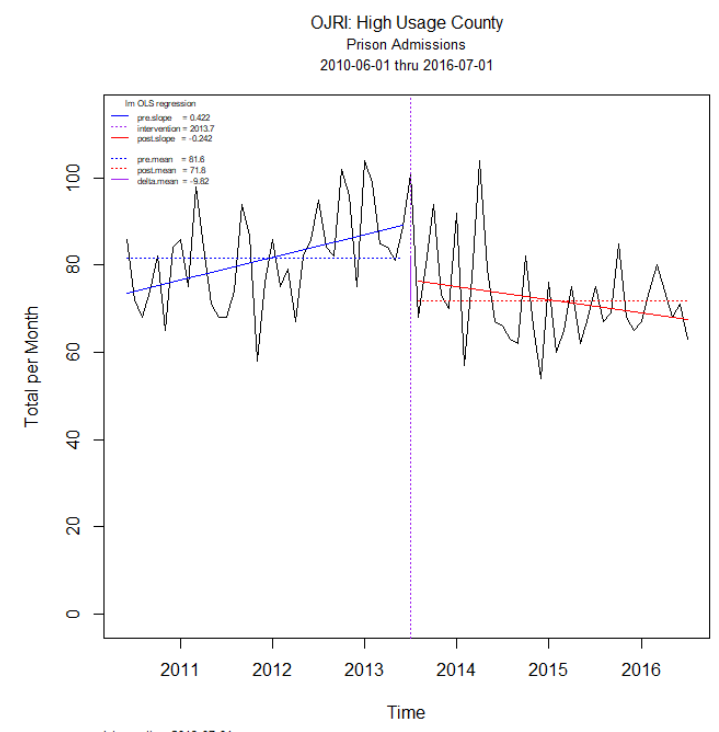

High Usage raw data with OLS

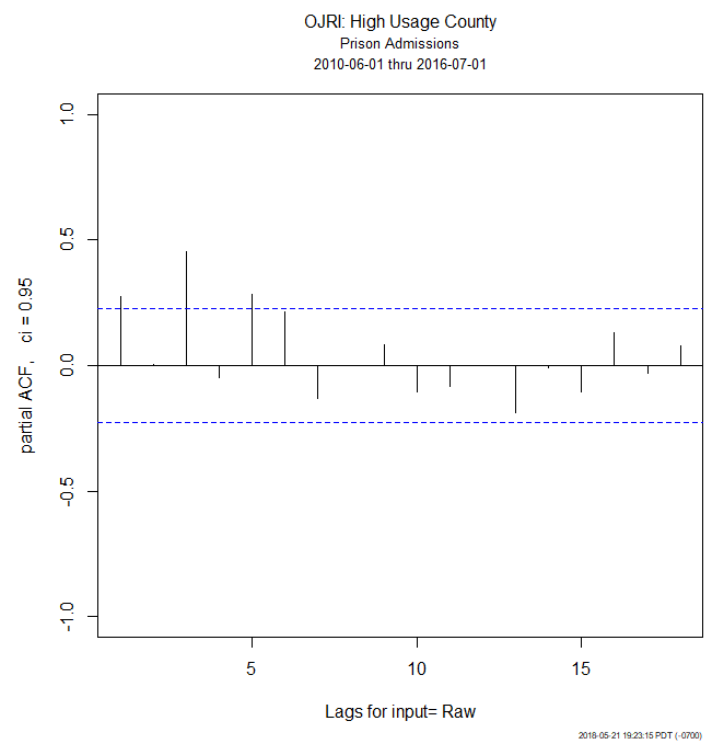

Partial ACF for raw data

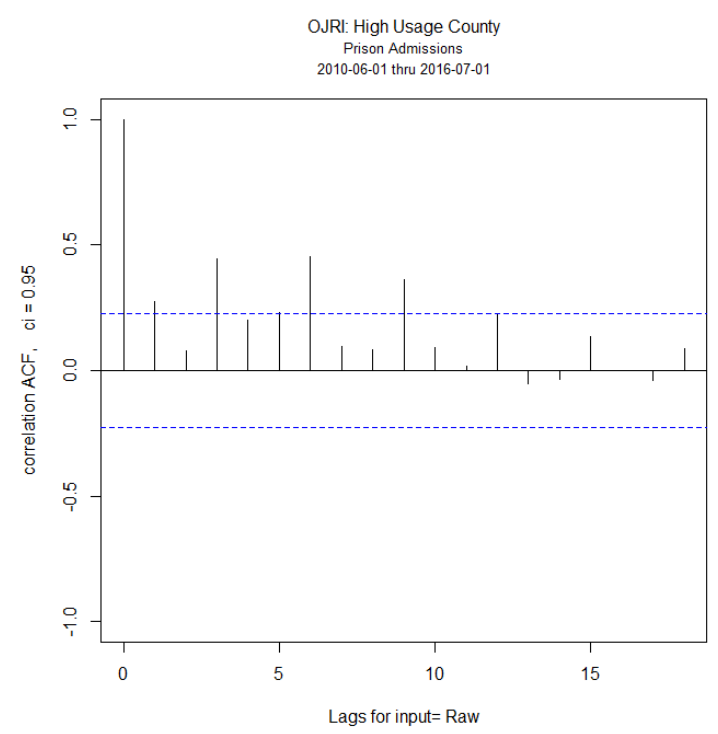

ACF plot for raw data

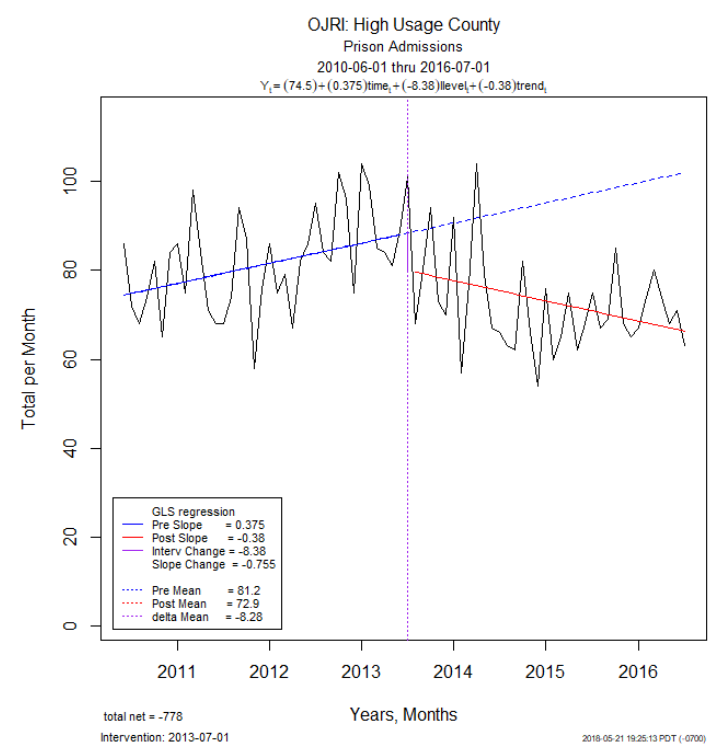

Final model fitted by GLS 


\section{Probation Admissions}

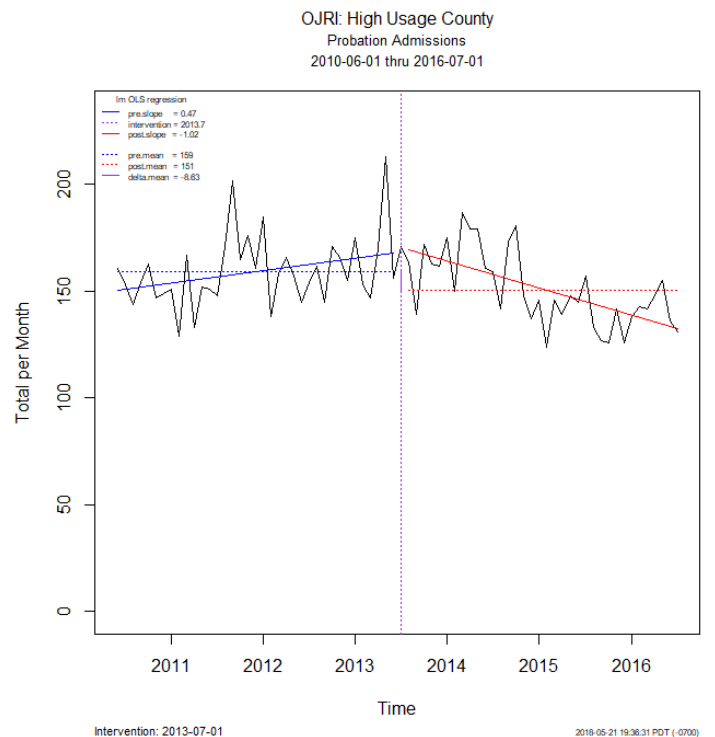

High Usage raw data with OLS

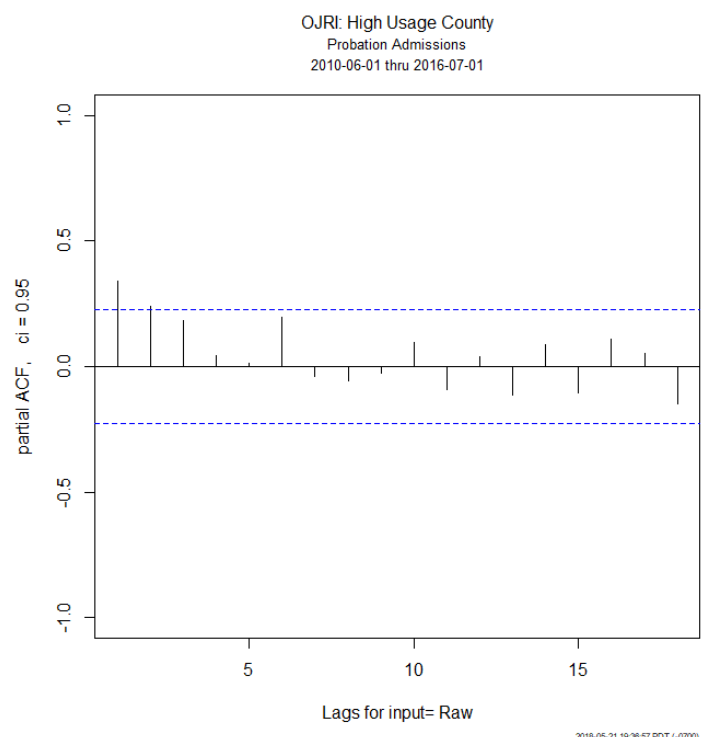

Partial ACF for raw data

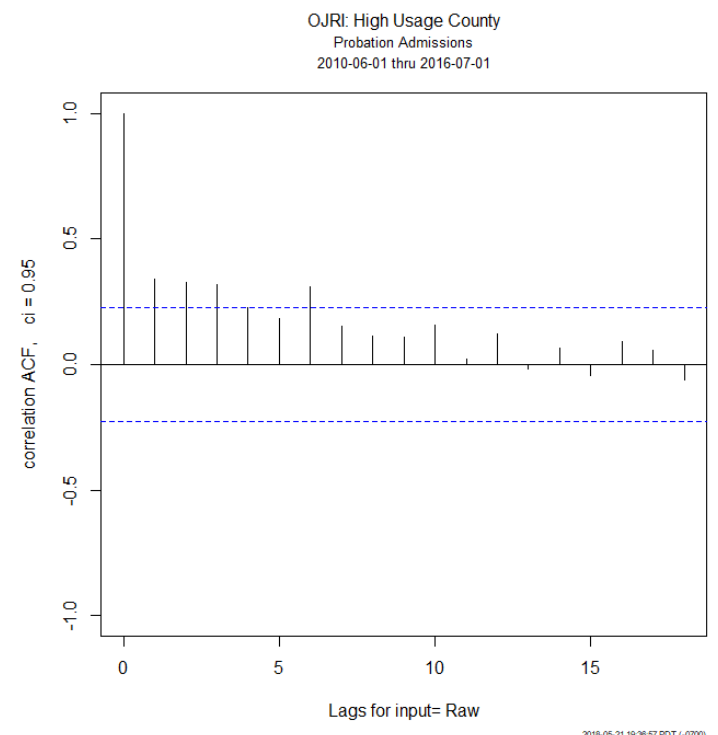

ACF plot for raw data

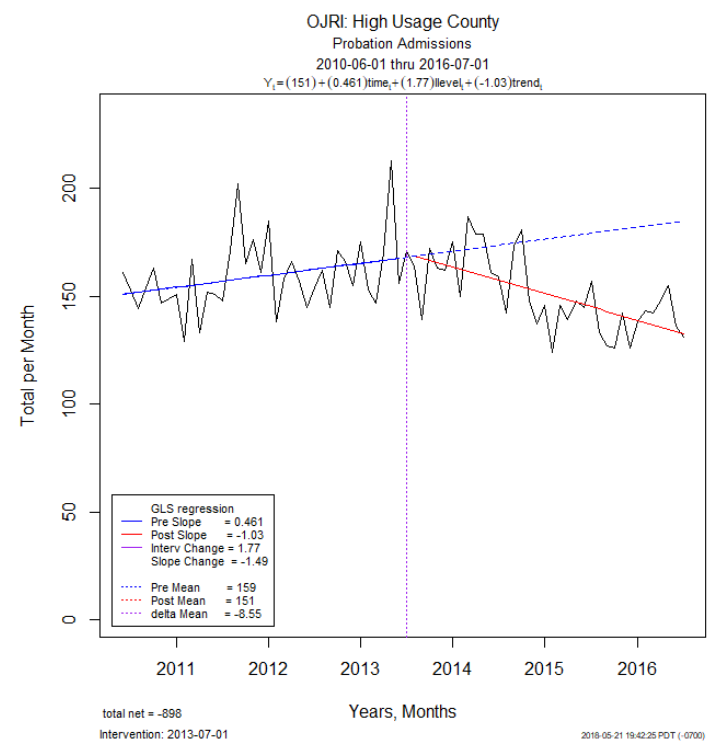

Final model fitted by GLS 
Community Supervision

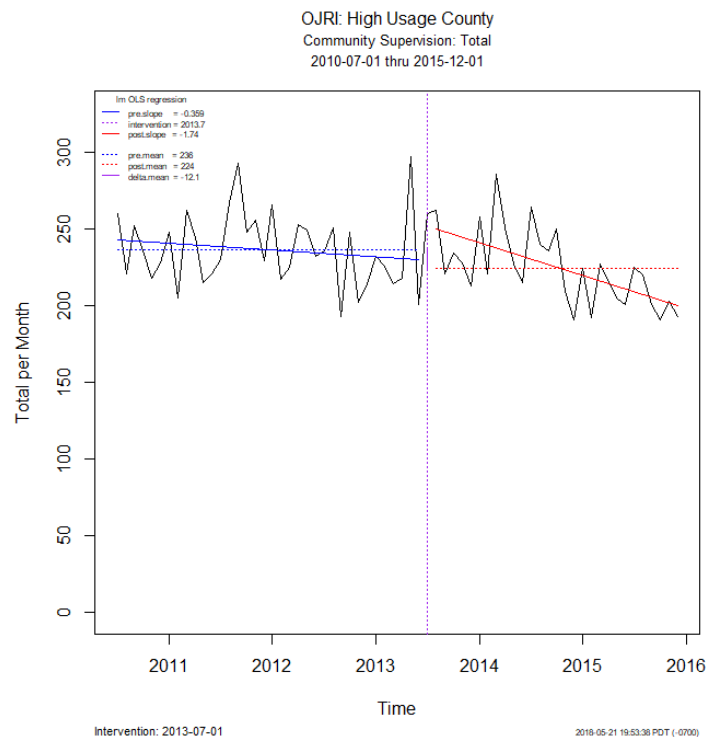

High Usage raw data with OLS

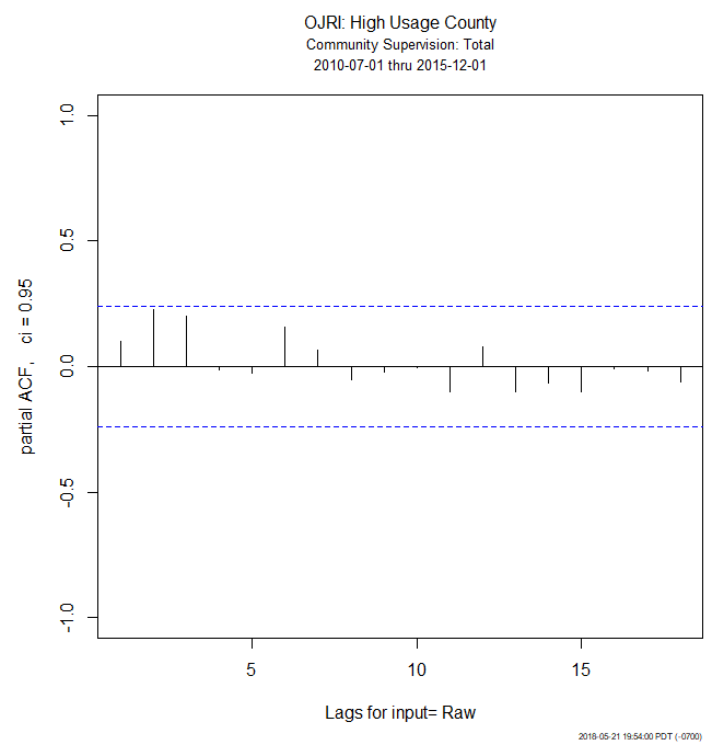

Partial ACF for raw data

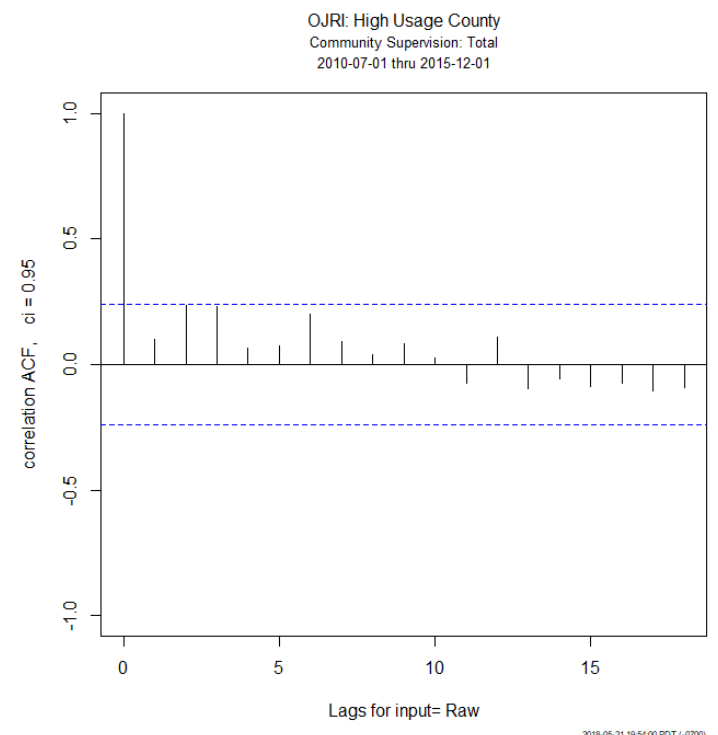

ACF plot for raw data

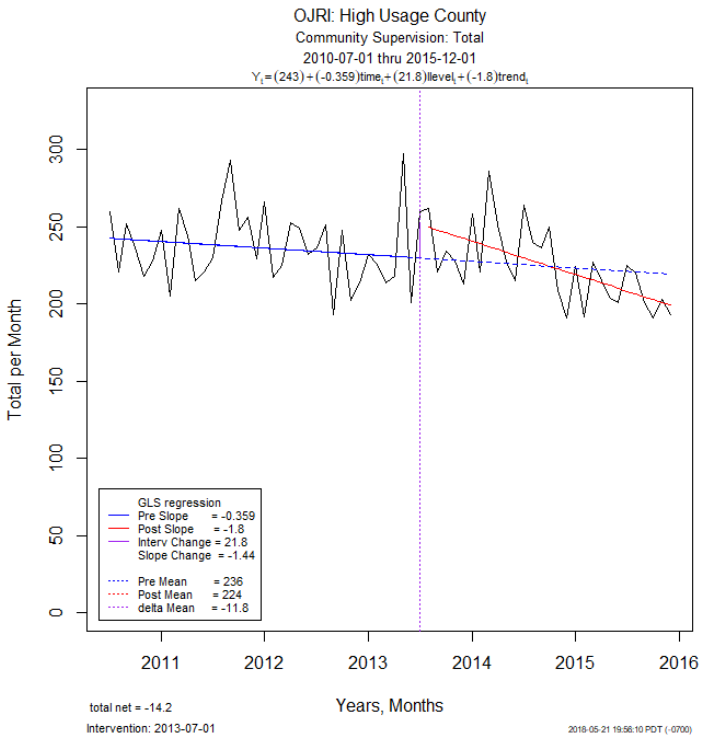

Final model fitted by GLS 
The Medium Usage County

Prison Admissions
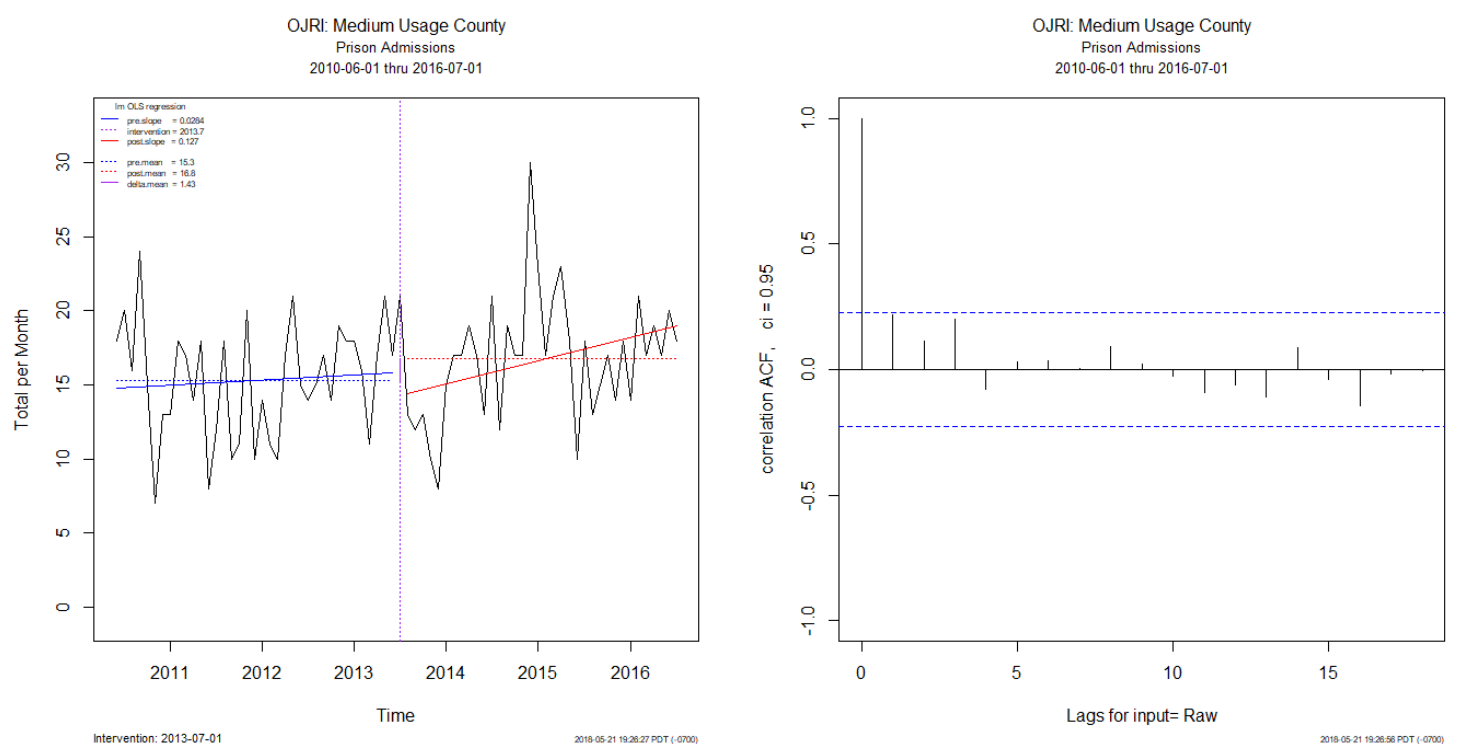

Medium usage proxy raw data with OLS
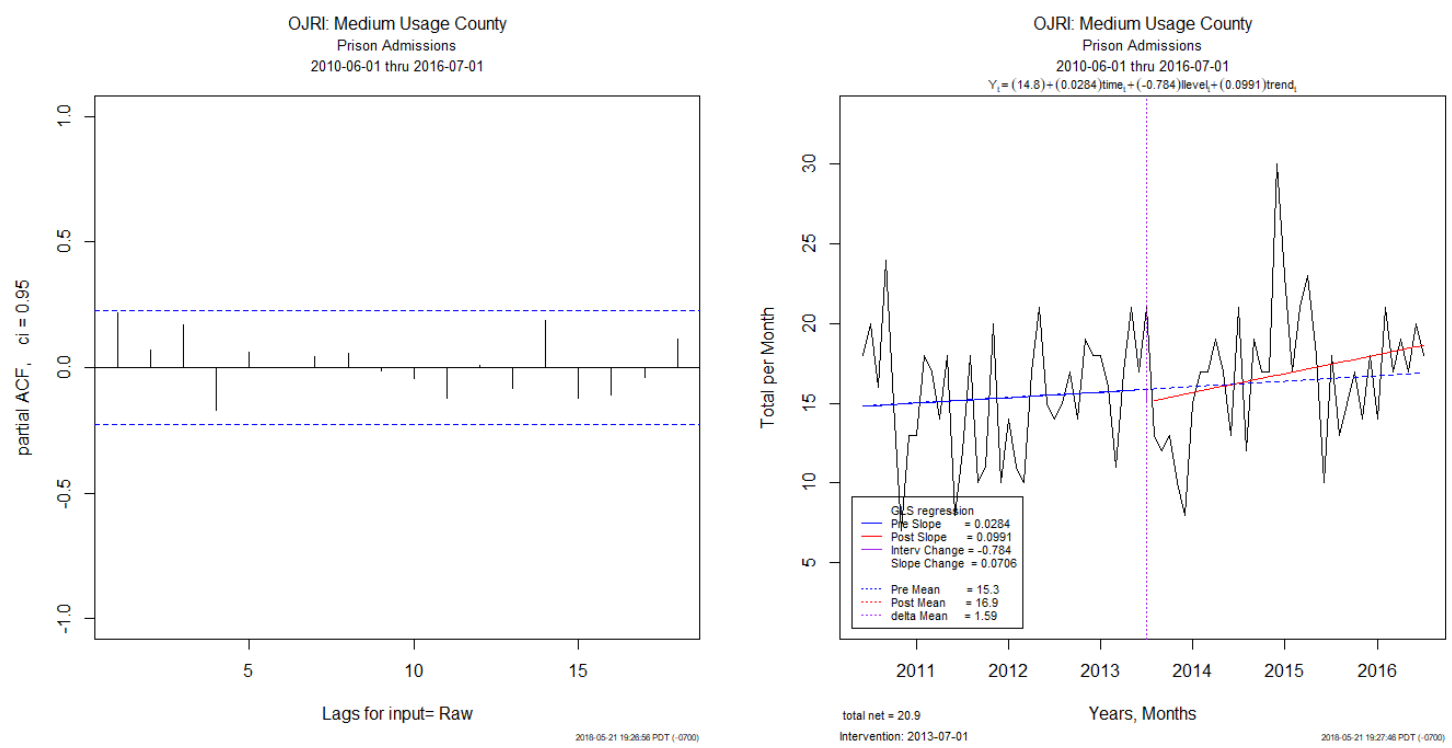

Partial ACF for raw data

Final model fitted by GLS 
Probation Admissions
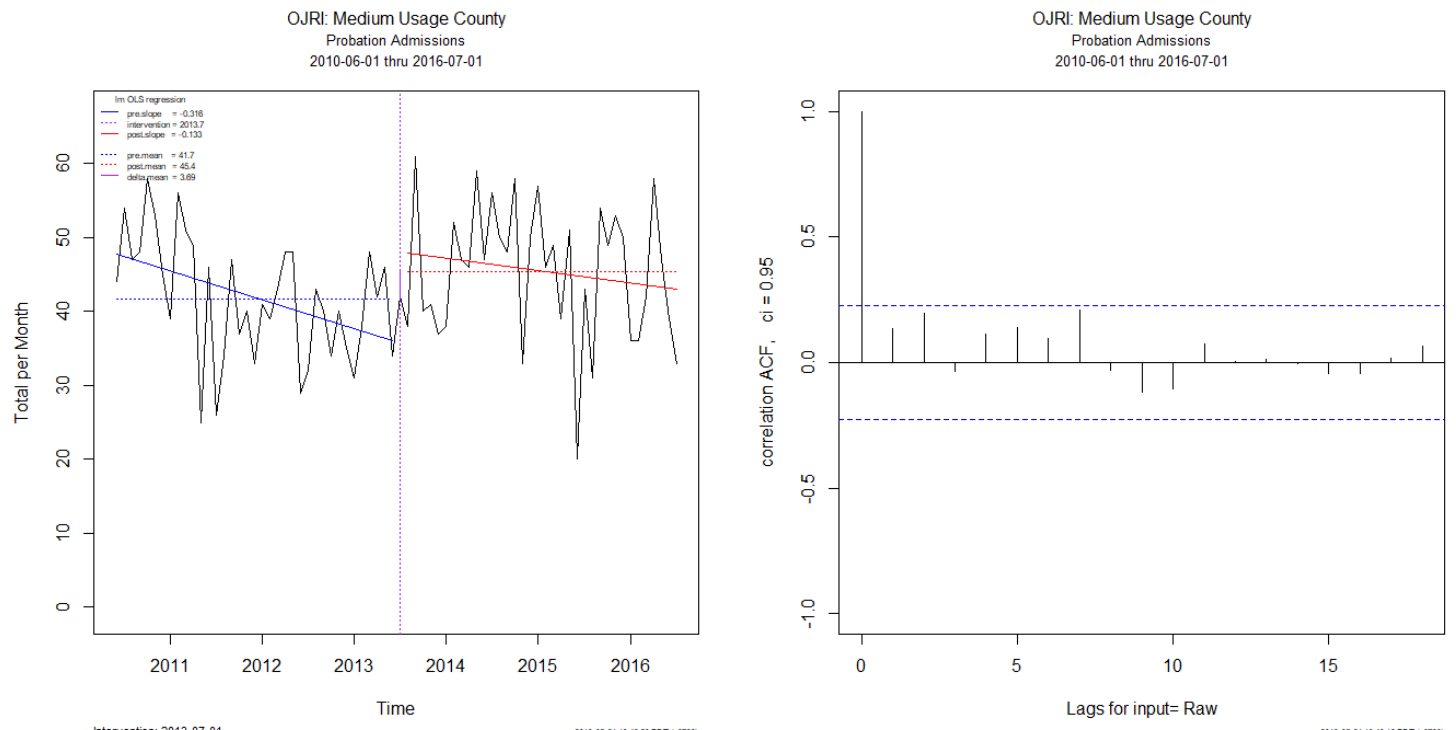

Medium usage proxy raw data with OLS

ACF plot for raw data
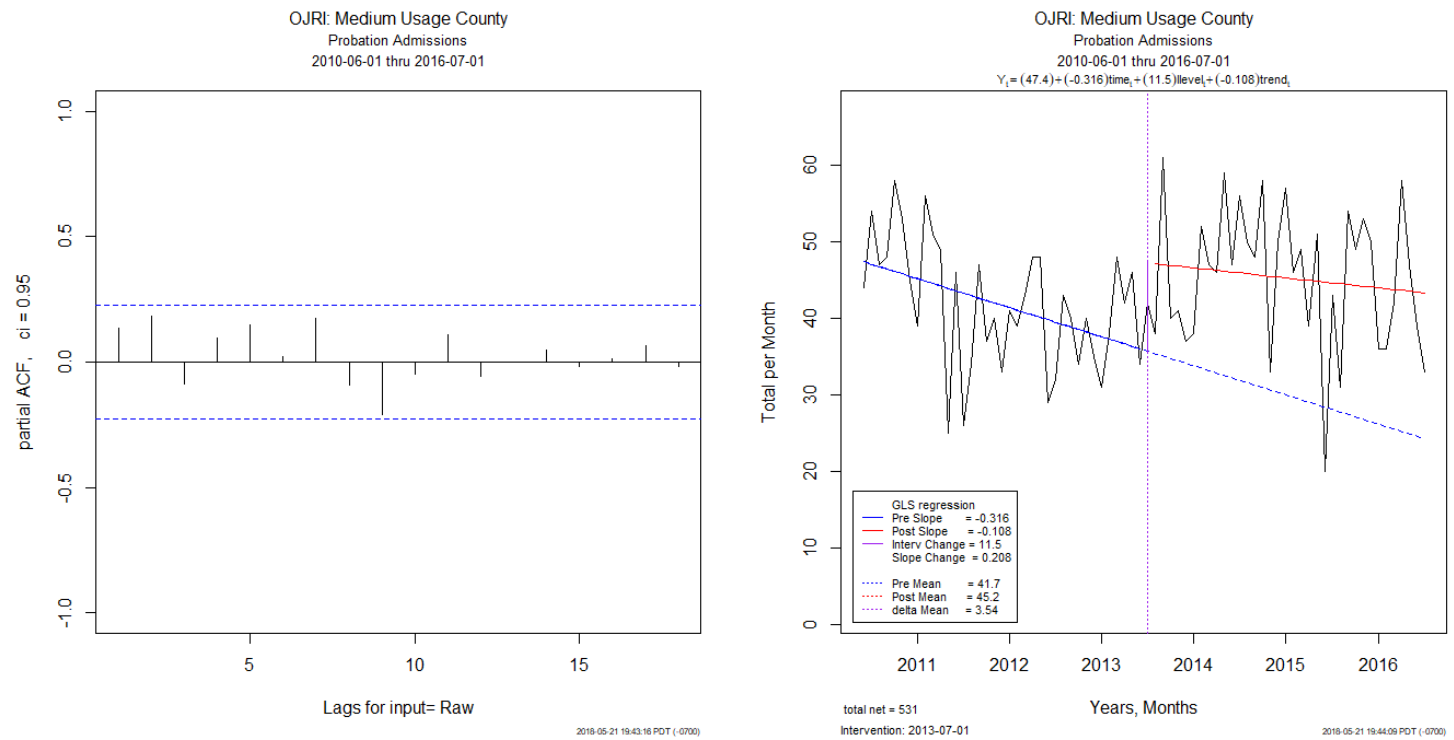

Partial ACF for raw data

Final model fitted by GLS 
Community Supervision

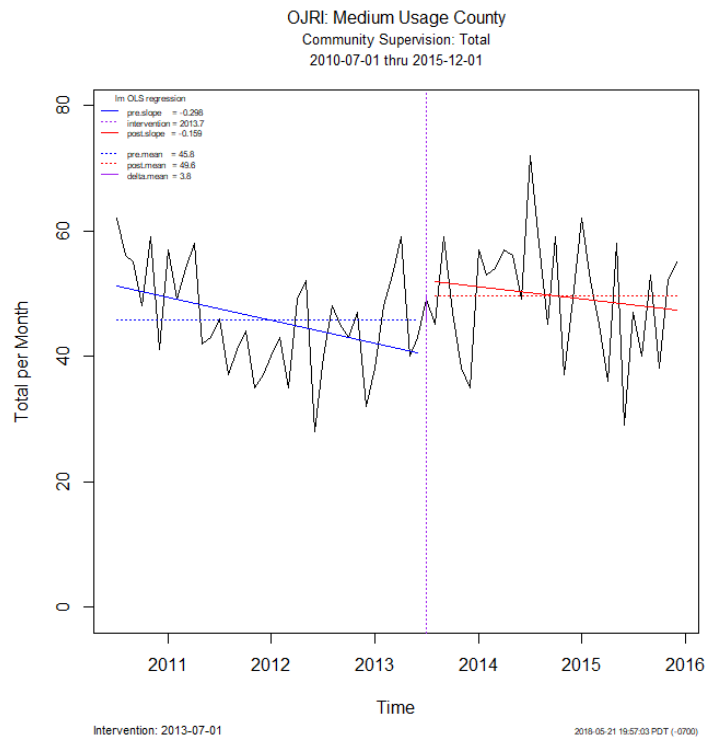

Medium usage proxy raw data with OLS

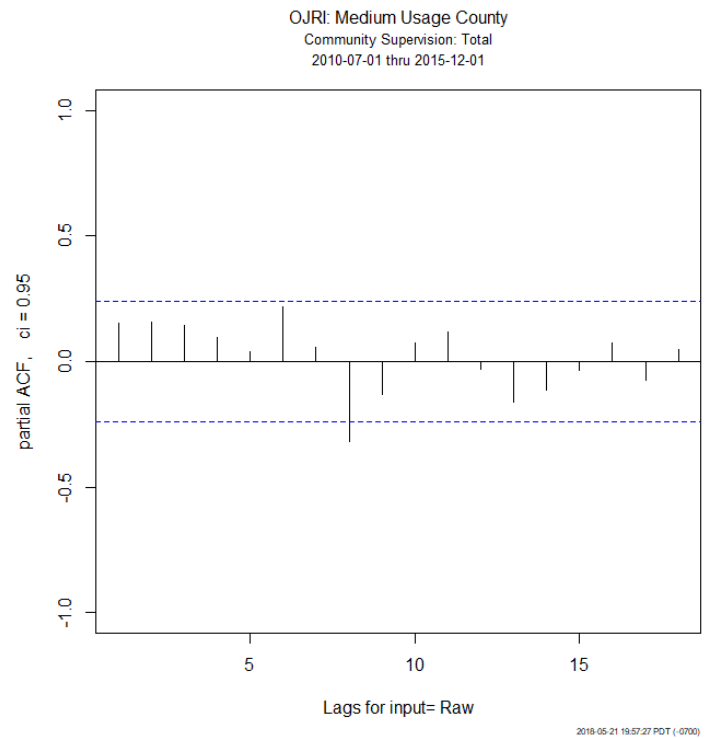

Partial ACF for raw data

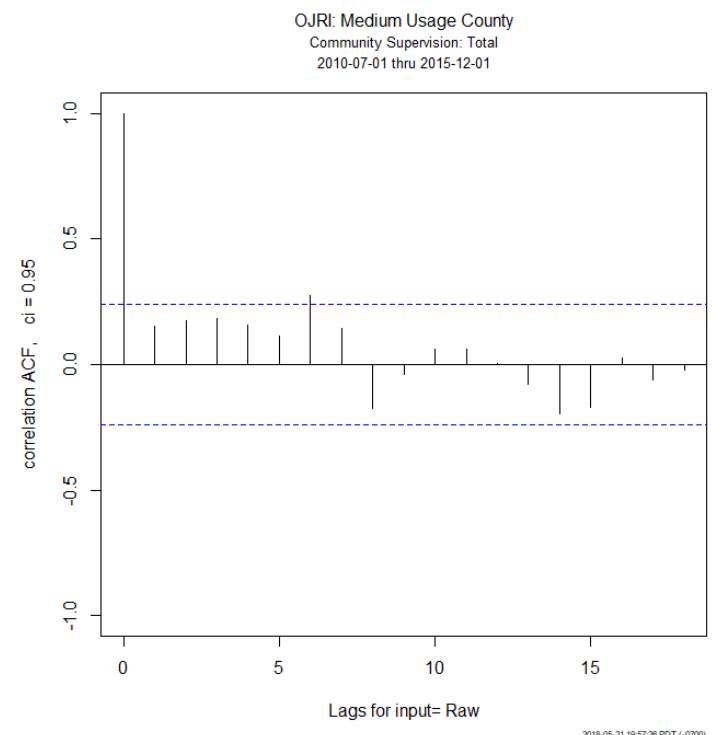

ACF plot for raw data

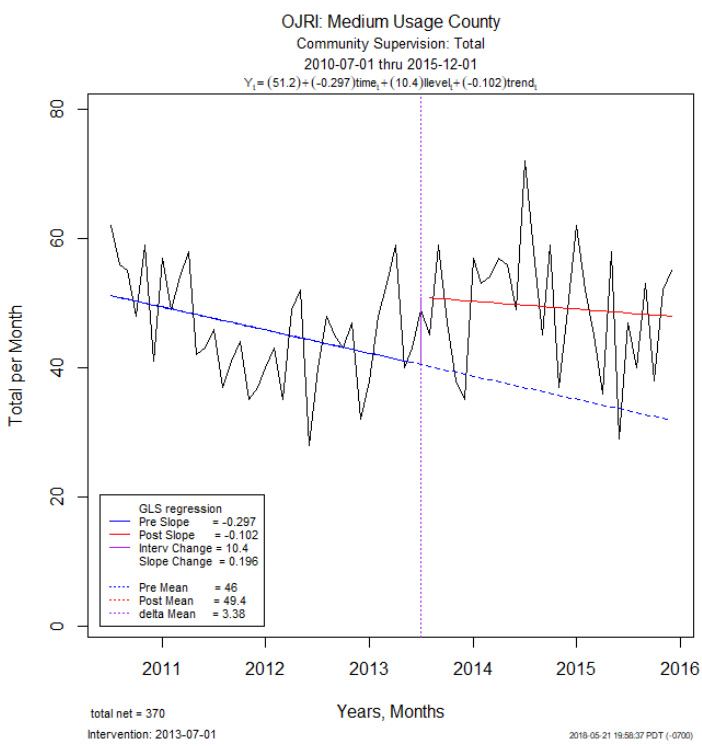

Final model fitted by GLS 
Low Usage County

Prison Admissions

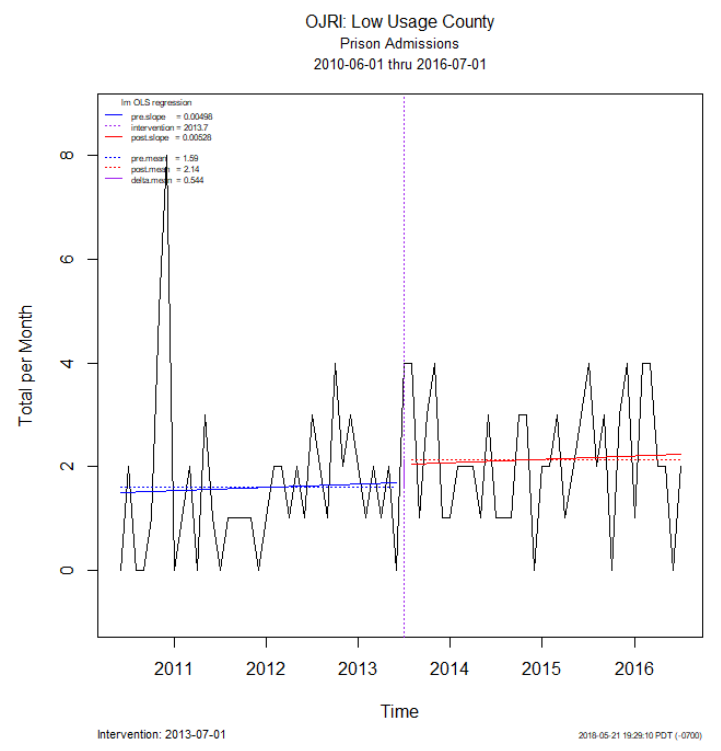

Low usage proxy raw data with OLS

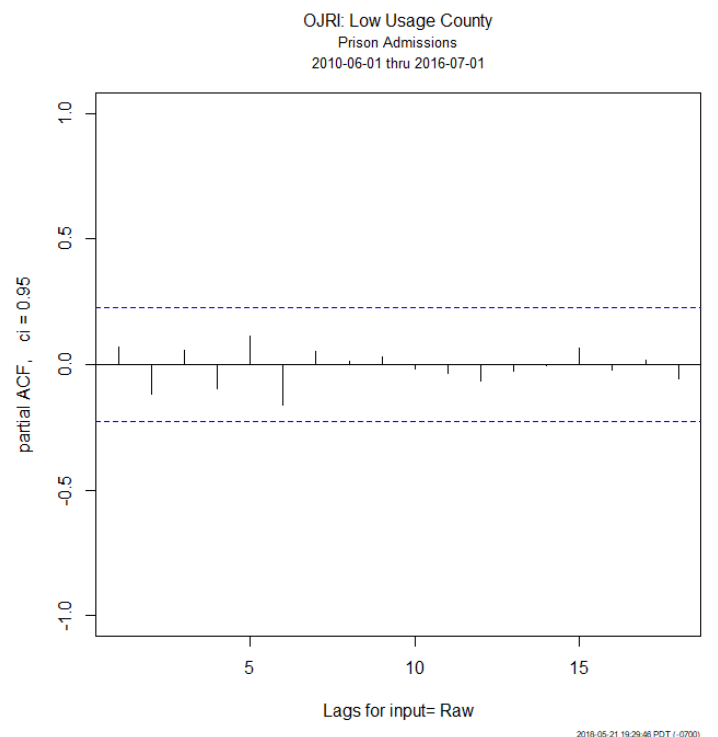

Partial ACF for raw data

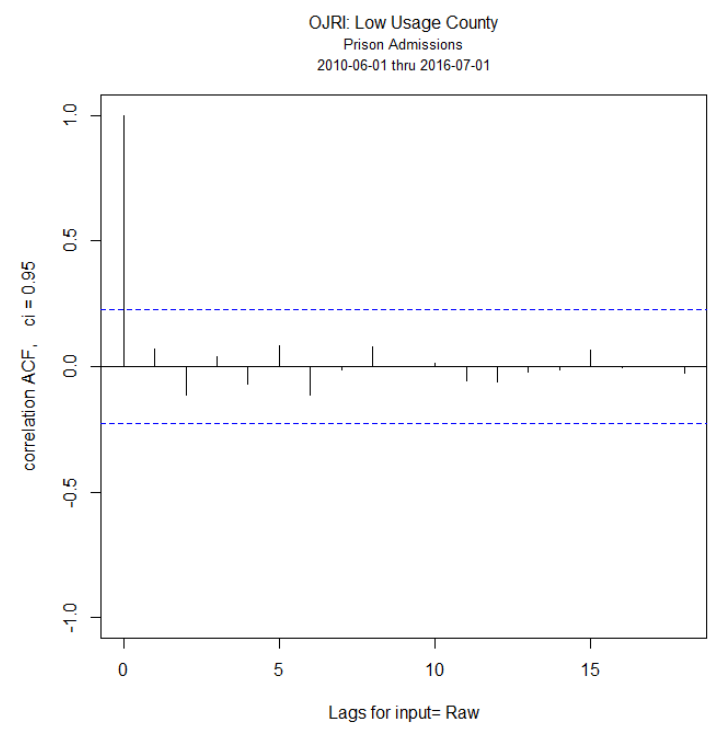

ACF plot for raw data

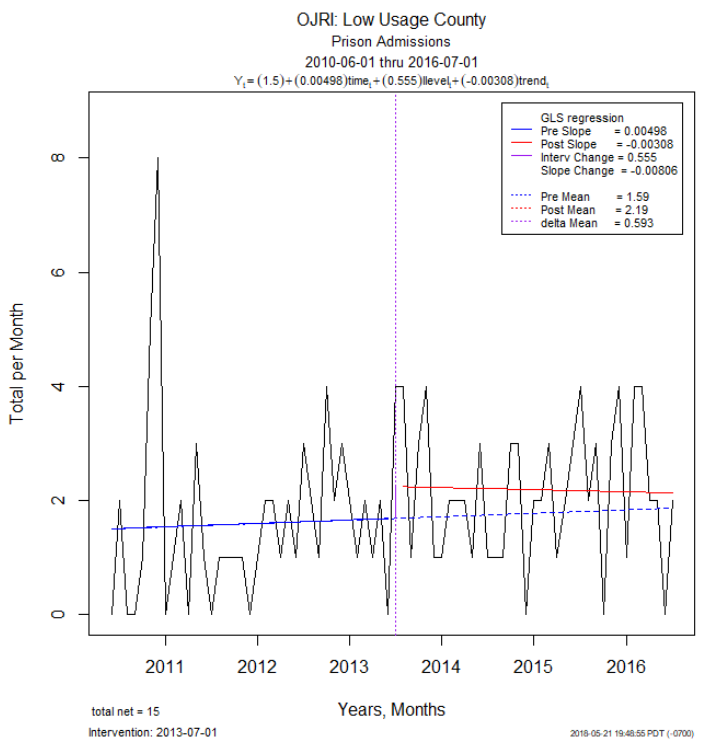

Final model fitted by GLS 
Probation Admissions

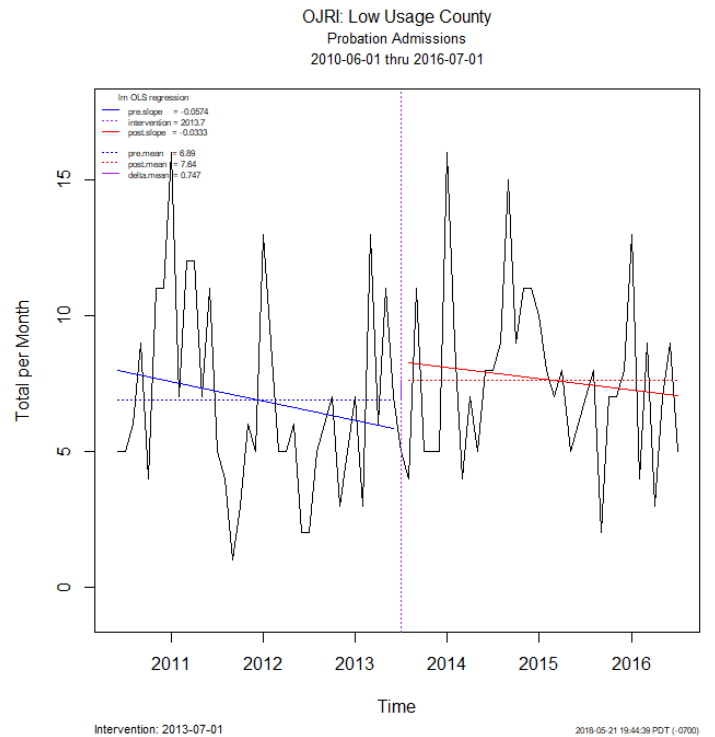

Low usage proxy raw data with OLS

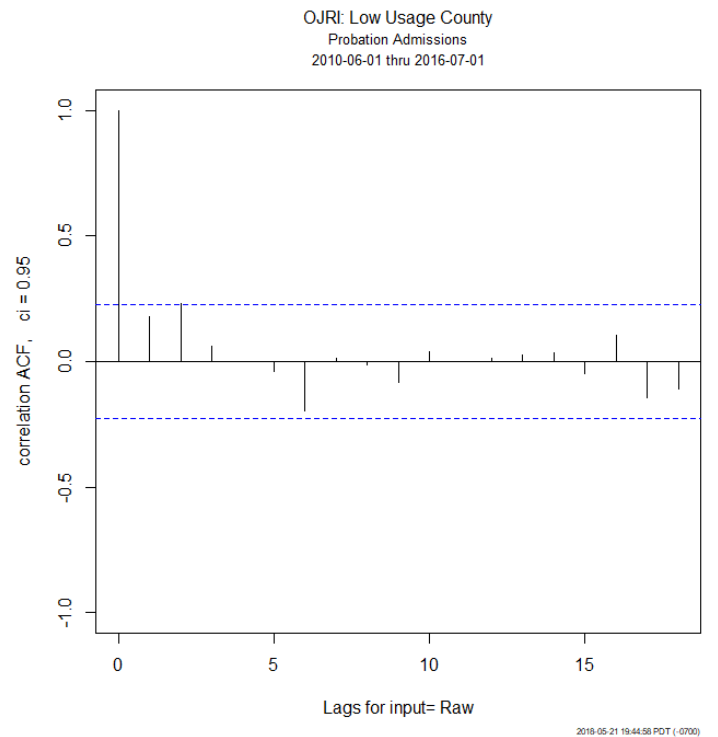

Partial ACF for raw data

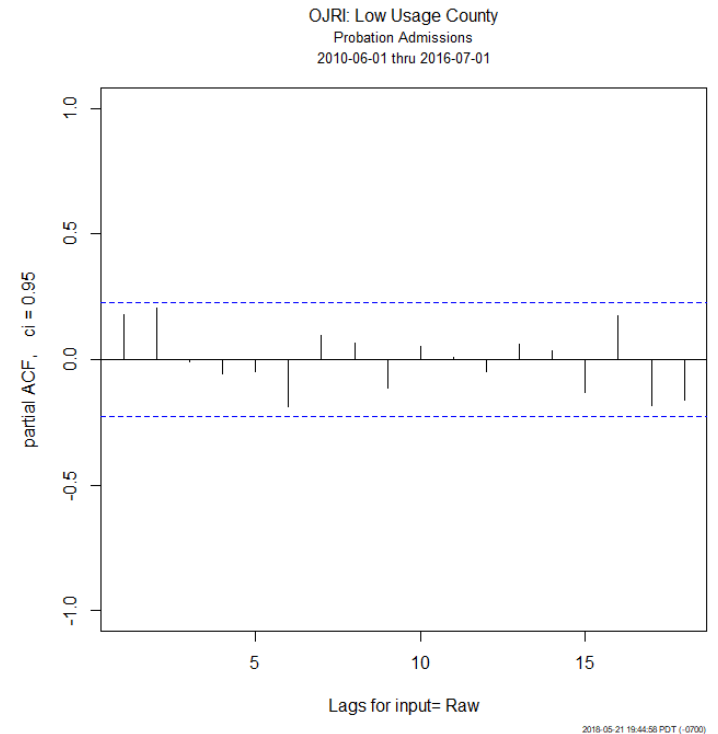

ACF plot for raw data

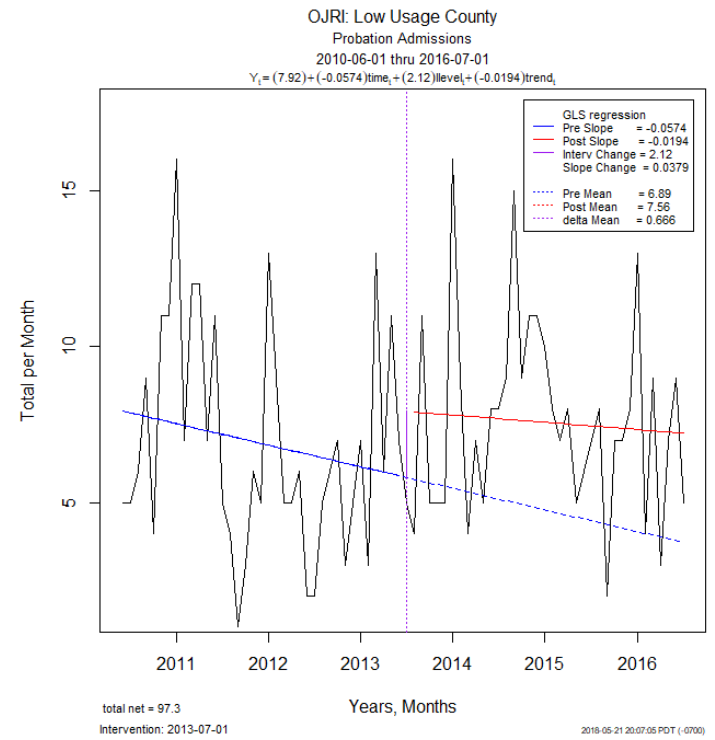

Final model fitted by GLS 
Community Supervision

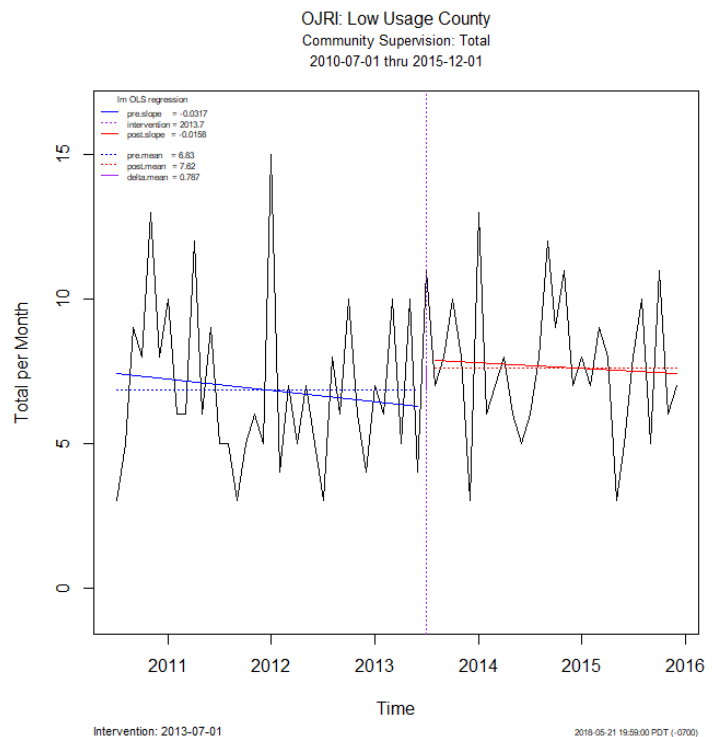

Low usage proxy raw data with OLS

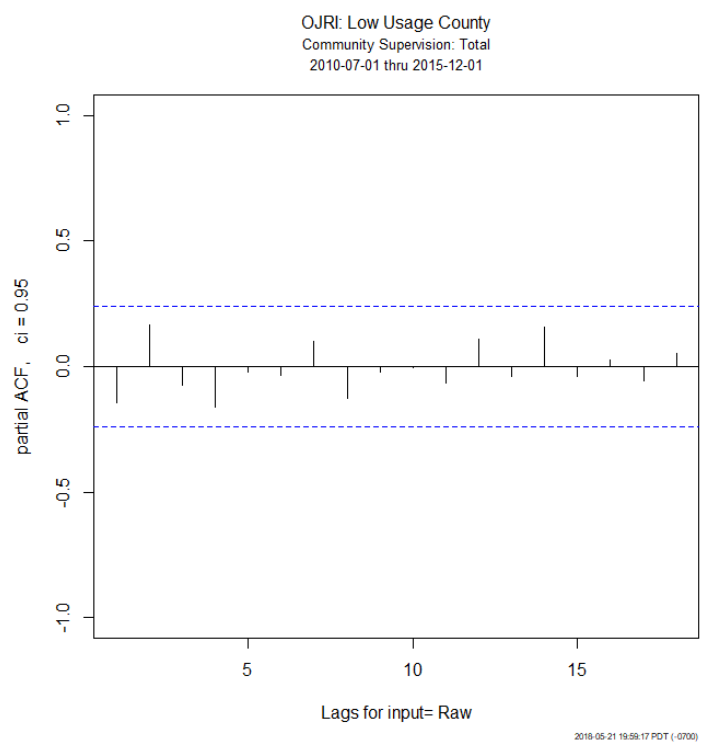

Partial ACF for raw data

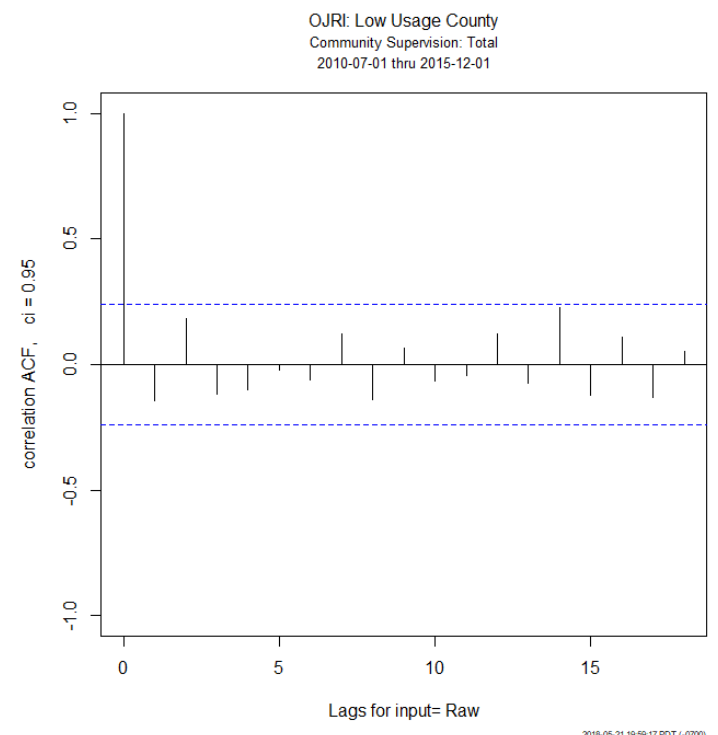

ACF plot for raw data

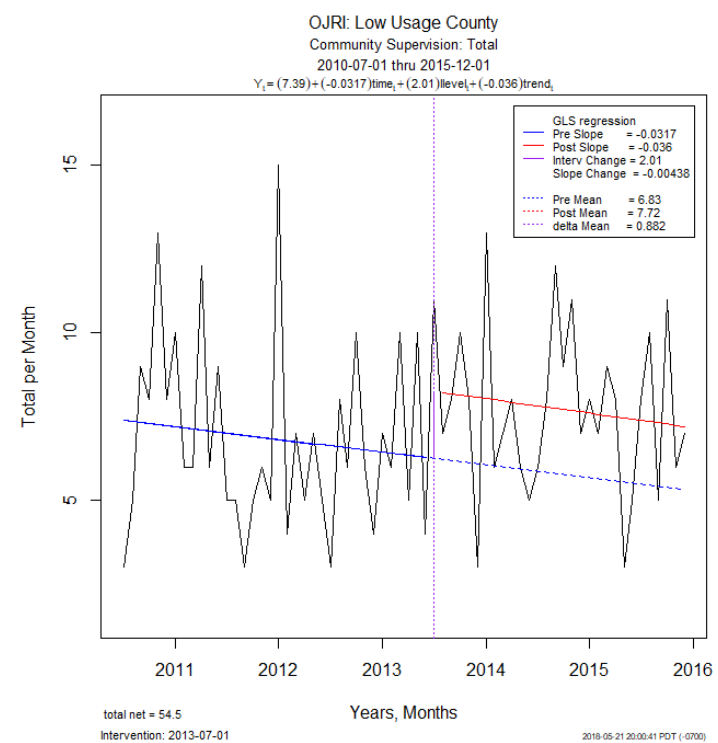

Final model fitted by GLS 


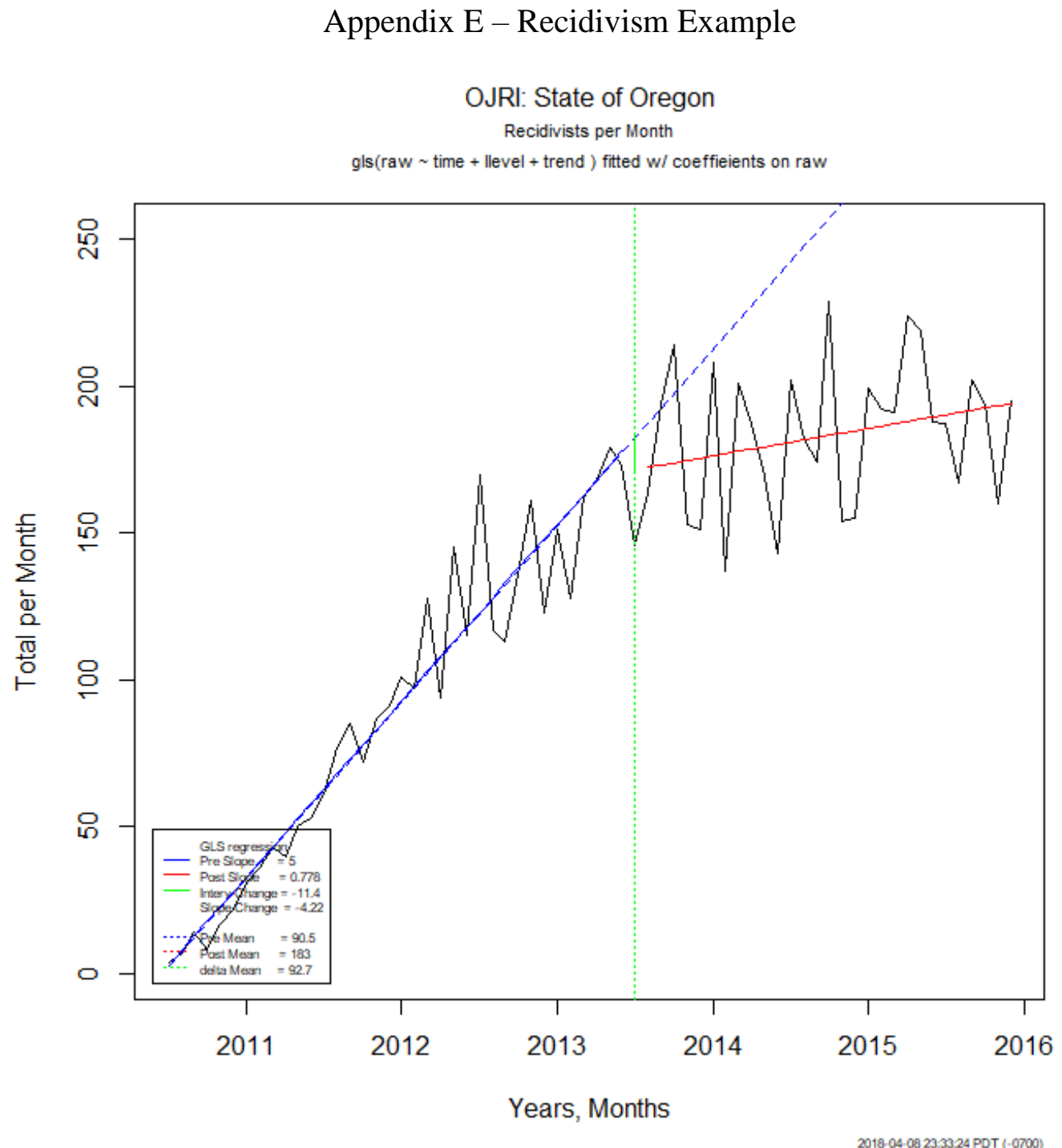

Example of recidivism rate data with phase in period. 